\title{
Charge Transport in BOTTOM-UP INORGANIC-ORGANIC AND QUANTUM-COHERENT NANOSTRUCTURES
}

Ksenia S. MAKaRENKo 


\section{Charge Transport in \\ BOTTOM-UP INORGANIC-ORGANIC AND \\ Quantum-Coherent Nanostructures}





\title{
Charge Transport in \\ BOTTOM-UP INORGANIC-ORGANIC AND \\ Quantum-Coherent Nanostructures
}

\section{DISSERTATION}

\author{
to obtain \\ the degree of doctor at the University of Twente, \\ on the authority of the rector magnificus, \\ prof. dr. H. Brinksma, \\ on account of the decision of the graduation committee, \\ to be publicly defended \\ on Friday 22 May 2015 at 12.45
}

by

Ksenia Sergeevna MAKARENKO

born on $11^{\text {th }}$ February 1987

in Taganrog, USSR 
This dissertation has been approved by the promotor:

Prof. dr. ir. W. G. van der Wiel

\section{Committee members:}

Prof. dr. P. M. G. Apers

Prof. dr. ir. W. G. van der Wiel

Chairman \& secretary

Prof. dr. ir. D. N. Reinhoudt

Assoc. Prof. dr. A. A. Golubov

University of Twente

University of Twente

University of Twente

Prof. dr. J. M. van Ruitenbeek

Leiden University

Prof. dr. E. Scheer

Universität Konstanz, Germany

Dr. E. Strambini

Scuola Normale Superiore di Pisa, Italy

\section{UNIVERSITY OF TWENTE.}

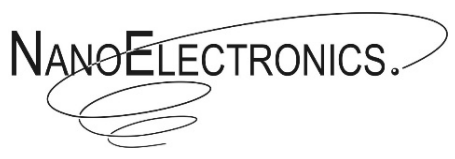

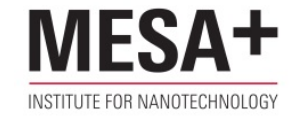

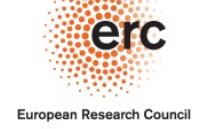

The research described within this thesis was carried out in the NanoElectronics Group at the MESA+ Institute for Nanotechnology at the University of Twente, Enschede, The Netherlands. European Research Council (ERC) Starting Grant no. 240433 finantially supported this research.

Published by Gildeprint Drukkerijen

ISBN: 978-90-365-3864-0

DOI: $10.3990 / 1.9789036538640$

Cover design: Ksenia S. Makarenko

Copyright (C) 2015 by Ksenia S. Makarenko

All rights reserved. No part of the material protected by this copyright notice may be reproduced or utilized in any form or by any means, electronic or mechanical, including photocopying, recording or by any information storage and retrieval system, without permission from the publisher.

Printed in The Netherlands, 2015 
... to my family...

...моей семье ... 



\section{Contents}

Chapter 1 INTRODUCTION ................................................................................ 1

1.1. Aharonov-Bohm Effect .............................................................................. 3

1.2. Universal Conductance Fluctuations ............................................................... 4

1.3. Coulomb Blockade .......................................................................................... 4

1.4. Charge Transport Through Organic Molecules ............................................ 8

1.5. Sample Fabrication and Measurements Techniques ............................ 10

1.6. Electrical Contacting of Nano Objects by Electron Beam Lithography

1.7. Design of Aharonov-Bohm Interferometers .......................................... 15

1.8. Low Noise Measurements .................................................................... 16

Chapter 2 NANOPARTICLE-BRIDGED MOLECULAR JUNCTIONS .................. 21

2.1. Dielectrophoresis Manipulation Method ................................................. 22

2.2. Device Fabrication and Experimental Setup ............................................ 23

2.3. Concentration, Time and Voltage Dependences .................................... 24

2.4. Current-Voltage Measurements through Molecular Monolayers in Nanoparticle Bridges........................................................................................... 26

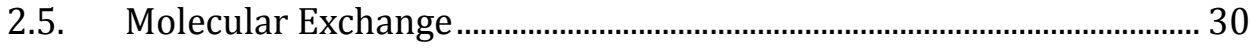

2.6. Single-Electron Transistor ...................................................................... 31

2.7. Evolution of the Designless Nanoparticle Network into Boolean Logic

Chapter 3 WEDGING TRANSFER TECHIQUE ……............................................... 37

3.1. Wedging Transfer as an Alternative Method for the Creation of Molecular Junctions .............................................................................................. 38

3.2. Device Fabrication.............................................................................. 40

3.3. Molecular Length Dependence.................................................................. 42

3.4. Comparison of the Molecular Junctions Made via Nanoparticle Bridge to the Molecular Junctions Created via Wedging Transfer Technique ............ 44

Chapter 4 BOTTOM-UP INORGANIC-ORGANIC SINGLE-ELECTRON TRANSISTORS 
4.1. Self-Assembly of a Single Nanoparticle to two Gold Nanorods .......... 48

4.2. Sample Fabrication................................................................................ 50

4.3. Electron Transport through Bottom-up Self-Assembled SingleElectron Transistors ............................................................................................. 52

4.4. Molecular Exchange ................................................................................ 57

Chapter 5 ELECTRON TRANSPORT THROUGH A PAIR OF METALLIC COULOMB ISLANDS COUPLED IN PARALLEL ………....................................... 63

5.1. Double Coulomb Islands Coupled in Parallel ............................................. 64

5.2. Electron Transport through a Pair of Coulomb Islands Coupled in

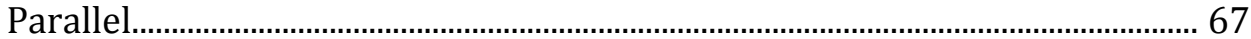

5.3. Simulation Results.................................................................................. 71

Chapter 6 DYNAMICAL NONLOCALITY IN A DIFFUSIVE QUANTUM INTERFEROMETER ..................................................................................... 77

6.1. Dynamic Nonlocality in a Diffusive Aharonov-Bohm Interferometer 79

6.2. Local and Nonlocal Measurements in the Multi-Terminal Quantum

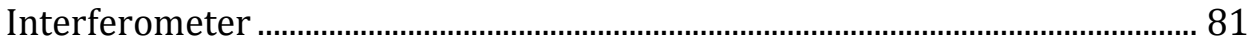

6.3. Analytical Model Explaining the Nonlocal Effect...................................... 83

6.4. Characterisation of Aharonov-Bohm Interferometers ........................... 85

Chapter 7 COHERENT ELECTRON TRANSPORT THROUGH HYBRID AHARONOV-BOHM INTERFEROMETERS......................................................... 93

7.1. Coherent Transport through Organic Molecular Layers ....................... 94

7.2. Aharonov-Bohm Oscillations in Gold Rings Bridged by Gold Nanoparticles................................................................................................ 94

7.3. Interference Effects in Aharonov-Bohm Ring Created via Selective Chemical Interaction ....................................................................................... 96

7.4. Hybrid Aharonov-Bohm Interferometers: Perspectives .....................101

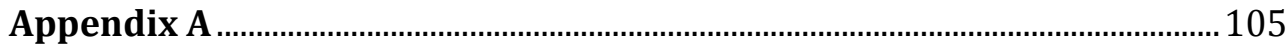

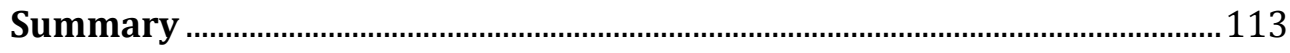

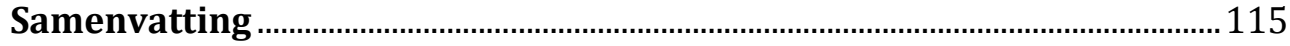

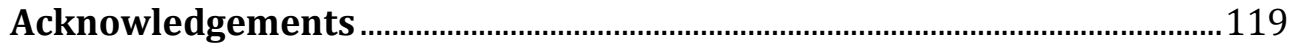




\section{Chapter 1 InTRODUCTION}

"An Experiment, like every other event which takes place, is a natural phenomenon; but in a Scientific Experiment the circumstances are so arranged that the relations between a particular set of phenomena may be studied to the best advantage".

James Clerk Maxwell, 1876 
The semiconductor electronics industry is showing a dramatic downscaling of the size of electronic devices driven by scientific and technological innovations [1]. If this scaling continues down to one molecule as an individual logic or memory unit hybrid electronics (where inorganic and organic molecular materials are combined [2]), in an irrevocable connection with quantum-mechanical phenomena (usually coming into view at nanoscale and at the temperatures close to absolute zero), could play a major role for the future of electronics. In fact, the potential of molecular electronics for applications such as diodes and memories has already been demonstrated [3].

The relatively young field of mesoscopic physics forms a bridge between the macroscopic world of bulk materials and the microscopic world of atoms and molecules and explores semiconducting, metallic and superconducting systems with typical dimensions in the range of 0.01-10 $\mu \mathrm{m}$. Mesoscopic physics is interested in a big variety of questions. What is a boundary condition of the size of a piece of material that "averages away" the wave-nature of a particle, governed by quantum mechanics, and "recover" its classical behaviour? If there are only a few electrons available, how will they behave? And many more...

"It seems as though we must use sometimes the one theory and sometimes the other, while at times we may use either. We are faced with a new kind of difficulty. We have two contradictory pictures of reality; separately neither of them fully explains the phenomena of light, but together they do"

Albert Einstein and Leopold Infeld, 1938

In this thesis, we use diffusive systems as inorganic components $\mathrm{Au}$ films, Au nanorods (NRs) and Au nanoparticles (NPs)), and self-assembled molecular layers as organic part in order to build hybrid devices. Chapter 1 gives a brief introduction to the charge transport through molecular layers and to the main phenomena in the diffusive regime. These are the AharonovBohm (AB) effect, universal conductance fluctuations (UCF), and the Coulomb blockade which reveal the quantum and classical behaviour of electrons. Chapter 1 also describes experimental methods and techniques required to open the secrets of electron transport. Chapters 2-3 are dedicated to the two different methods (dielectrophoresis and wedging transfer techniques, respectively) of the molecular junction fabrication and the consistent study of the charge transport mechanisms through organic molecular layers. A unique bottom-up approach of the fabrication of single-electron transistors (SETs) is described in detail in chapter 4 , followed by the investigation of the electron 
behaviour in a pair of metallic Coulomb islands coupled via molecular barriers in parallel (Chapter 5). Chapter 6 discusses dynamic nonlocality in a diffusive system where $A B$ effect is used as a switch to tune electron transport. In the end of the thesis (Chapter 7) we show an indication of the coherent electron transport in a hybrid inorganic-organic $A B$ interferometer with embedded molecular junctions and propose novel geometries of hybrid $A B$ rings for future studies of the coherence in organic molecules.

\subsection{Aharonov-Bohm Effect}

In 1959, Yakir Aharonov and David Joseph Bohm formulated a Gedanken Experiment describing how a magnetic flux affects the interference of a split electron wave (Fig. 1.1) [4]. They suggested that if a single-electron wave is split into two partial waves enclosing a flux localised in the area between the two waves, such that no magnetic field $\mathrm{B}$ exists anywhere along the path of the electron wave, the vector potential A associated with field cannot be taken equal to zero and still effects the phase of the electron wave. Similarly, an electrostatic potential $V$ contributes to the phase even in absence of an electric field E.

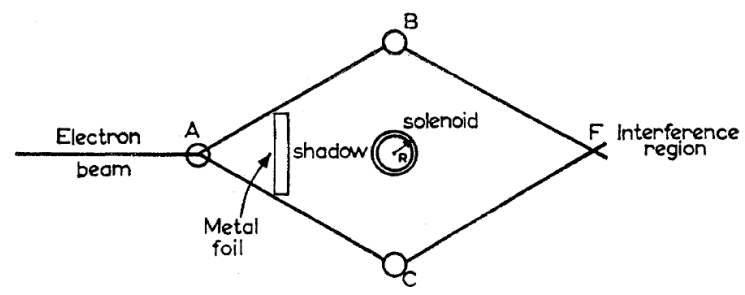

Figure 1.1. Schematic experiment to demonstrate single-electron interference with a time-independent magnetic vector-potential [4].

The phase $\varphi$ acquired by the electron wave, while travelling along the path, is given by:

$$
\varphi=\frac{2 \pi}{h} \int(m \mathrm{v}+|e| \mathrm{A}) \cdot \mathrm{d} s=2 \pi \frac{|e|}{h}\left[\int V d t+\int \mathrm{A} \cdot \mathrm{d} s\right],
$$

where $h$ is the Planck constant, $e$ is the elementary charge, $s$ is a completed path and $m$ and $v$ are electron mass and velocity, respectively. The phase difference between the two paths is:

$$
\Delta \varphi=\varphi_{1}-\varphi_{2}=2 \pi \frac{|e|}{h}\left[\int_{0}^{t_{0}} \Delta V d t+\oint \mathrm{A} \cdot \mathrm{d} l\right]=2 \pi \frac{|e|}{h} \Delta V t_{0}+2 \pi \frac{|e|}{h} B S,
$$




$$
\begin{aligned}
\Delta \varphi_{\Delta \mathrm{V}} & =2 \pi \frac{|e|}{h} \Delta V t_{0}, \\
\Delta \varphi_{\mathrm{A}} & =2 \pi \frac{|e|}{h} B S,
\end{aligned}
$$

where $\Delta V$ is the electrostatic potential difference between two paths, $t_{0}$ is the time between splitting and recombination of the single-electron wave, $S$ is the enclosed area by two paths, $\Delta \varphi_{\Delta V}$ and $\Delta \varphi_{\mathrm{A}}$ are contributions to the phase difference by the electrostatic and magnetic potentials, respectively. The first experimental observation of the Aharonov-Bohm effect in a single diffusive metal ring was performed in 1985 by Webb et al [5]. The importance of the $\mathrm{AB}$ effect is underlined by the qualification "one of the seven wonders of the quantum world" by the New Scientist magazine [6].

\subsection{Universal Conductance Fluctuations}

Universal conductance fluctuations are observed in coherent mesoscopic systems during electrical measurements at low temperatures and originated from a magnetic flux, piercing the leads in the device [7-9]. The physical origin of UCF is the same as of the AB effect, interference. Due to the artificially fixed geometry of the path, which electron wave function takes in a $\mathrm{AB}$ interferometer, $\mathrm{AB}$ signal has a periodic character in magnetic field with a period of $\Delta B=h /$ enS (Eq.1.4) (where $n$ is a harmonic number). While UCF take place in the wires and leads of the device, where there is no well-defined $\mathrm{AB}$ path, so that the correlated field is limited by the size of the device and the coherence length. Therefore, the effect is rather random leading to the appearance of the aperiodic fluctuations. UCF will appear in any (semi-) conducting systems independent of the sample size and degree of disorder [7], when the $B$-field goes through the device.

$\mathrm{AB}$ effect and UCF are governed by a magnetic flux penetrating the device and magnitudes of their fluctuations are both in the order of $e^{2} / h$. The period of $\mathrm{AB}$ oscillations is determined by the ring's enclosed area $S_{\text {in }}$, while UCF are correlated with the area of the arms $S_{\text {arm }}$. This leads to the fact that in order to distinguish AB effect from UCF the aspect ratio between $S_{\text {in }}$ and $S_{\text {arm }}$ should be $\gg 1$.

\subsection{Coulomb Blockade}

A Coulomb island is an isolated conductor that can be filled with electrons and perform single-electron transport (Fig. 1.2a) [10]. Coulomb islands have been shown to be useful systems to study a wide range of physical phenomena. In the following experiments we are using citrate (Chapter 2) and 
cetyltrimethylammonium bromide stabilized (Chapters 4,5) Au nanoparticles (NPs) with a diameter of $20 \mathrm{~nm}$ as Coulomb islands.

By applying small voltages at low temperatures it is possible to observe Coulomb blockade, an important phenomenon of the suppressed conductance through the Coulomb island. Figure 1.2a shows a schematic of the confined Coulomb island coupled via tunnel barriers to the three terminals: source, drain and gate electrodes. Source and drain provide a voltage drop across the Coulomb island resulting in the staircase current-voltage characteristic while the gate serves to change its electrostatic energy giving rise to the Coulomb oscillations in the current-voltage spectrum.

a

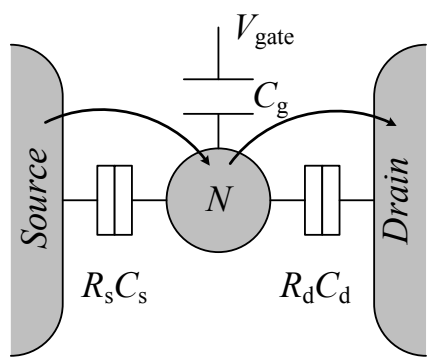

b

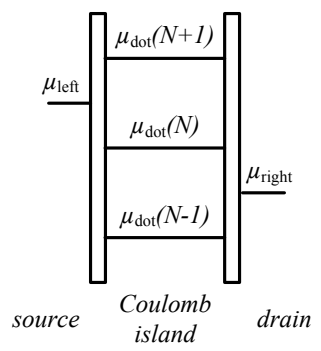

Figure 1.2. Schematics of (a) a confined Coulomb island and (b) electrochemical potentials $\mu_{\text {dot }}(N)$ in an island showing single-electron transport. $R_{\mathrm{S}}$ and $R_{\mathrm{d}}$ are tunnel resistances through source and drain, respectively.

The total number of electrons residing on the island is $N e$ (where $e$ is the elementary charge and $N$ is an integer number). When tunnelling occurs, the charge of the island changes by $e$. An addition of one elementary charge on the object will require a charging energy of $E_{C}=e^{2} / C$, where $C$ is the capacitance of the island. This capacitance is the sum of the capacitances between the dot and source $C_{\mathrm{s}}$, drain $C_{\mathrm{d}}$ and gate $C_{\mathrm{g}}: C=C_{\mathrm{s}}+C_{\mathrm{d}}+C_{\mathrm{g}}$. Charging energy becomes important when it dominates over the thermal energy $k_{B} T$ (where $k_{B}$ is the Boltzmann constant and $T$ is the temperature), and when barriers are sufficiently opaque such that electrons are located either in the source, in the drain, or on the island. Typical time to charge/discharge an island is $\Delta t=R_{\mathrm{t}} C$, where $R_{\mathrm{t}}$ is tunnel resistance of the barriers. From the Heisenberg uncertainty principle $E \Delta t=\left(e^{2} / C\right) R_{\mathrm{t}} C>h$ (where $h$ is the Planck constant) follows [11]:

$$
R_{\mathrm{t}} \gg h / e^{2}
$$




$$
e^{2} / C \gg k_{B} T
$$

The current through the Coulomb island is determined by the number of available states on the island that follows from the calculation of its electrochemical potential $\mu_{\text {dot }}(N)$. This is by definition the minimum energy to add one electron to an island:

$$
\mu_{\mathrm{dot}}(N) \equiv U(N)-U(N-1)=E_{N}+\frac{\left(N-N_{0}-1 / 2\right) e^{2}}{C}-e \frac{C_{g}}{C} V_{\text {gate }},
$$

where $U(N)$ is the total ground state energy for $N$ electrons on the island at zero temperature and at a gate voltage $V_{\text {gate }}, N_{0}$ is the number of electrons at zero gate voltage, $E_{\mathrm{N}}$ represents the chemical contribution $\mu_{\mathrm{ch}}(N)$. Electron transport through the island is possible only when $\mu_{\mathrm{dot}}(N)$ lies between electrochemical potentials of the source $\mu_{\text {left }}$ and drain $\mu_{\text {right }}: \mu_{\text {left }} \geq \mu_{\text {dot }} \geq$ $\mu_{\text {right }}$ (Fig. 1.2b). When at fixed gate voltage, the number of electrons is changed by one, the resulting change in electrochemical potential is:

$$
\Delta \mu_{\operatorname{dot}}(N)=\mu_{\text {dot }}(N+1)-\mu_{\text {dot }}(N)=E_{C}+\Delta E,
$$

where $\Delta E$ is the energy spacing between two discrete quantum levels.

Figure 1.3 shows a schematic stability diagram (Coulomb diamonds) of the electron transport through the Coulomb island as a function of the sourcedrain voltage $V_{\text {bias }}$ and $V_{\text {gate, }}$ where grey areas represent the suppressed conduction through the island.

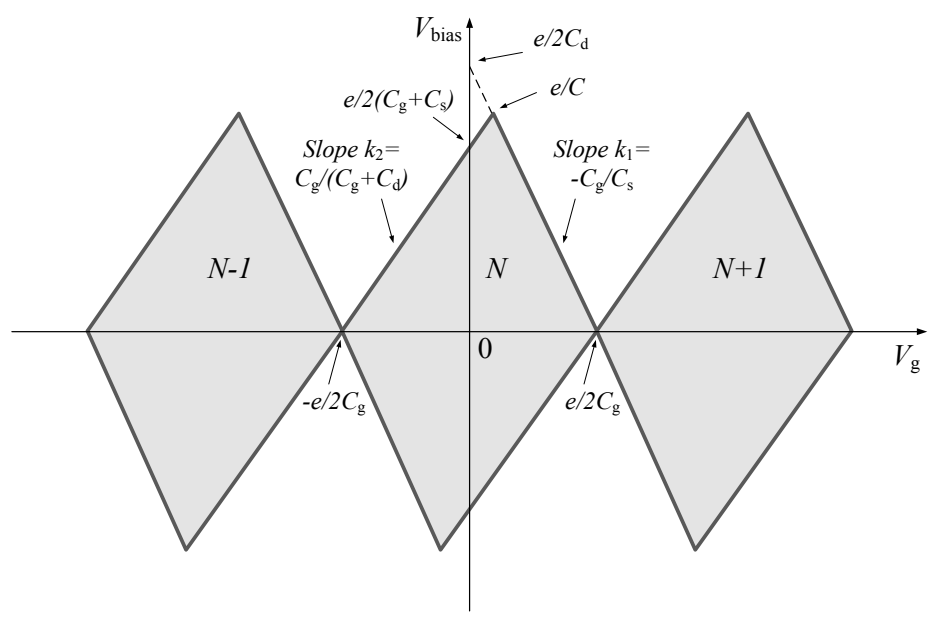

Figure 1.3. Stability diagram showing single-electron transport through the Coulomb island as a function of $V_{\text {bias }}$ and $V_{\text {gate, }}$ where grey areas represent the suppressed conduction through the island. From the slopes of the Coulomb diamonds important coupling parameters can be extracted. 
In the regime that thermal broadening $k_{B} T$ is greater than tunnelling broadening $h \Gamma$ (where $\Gamma=I / e$ is tunnelling rate and $I$ is the total current through barriers) [11], Coulomb blockade can be divided into two thermal regimes determined by the spacing between energy levels $\Delta E$ on the Coulomb island: classical (or metallic), when many levels are excited by thermal fluctuations $\left(\Delta E \ll k_{B} T \ll e^{2} / C\right)$, or quantum, when only one or a few levels participate in transport $\left(k_{B} T \ll \Delta E \ll e^{2} / C\right)[11]$.

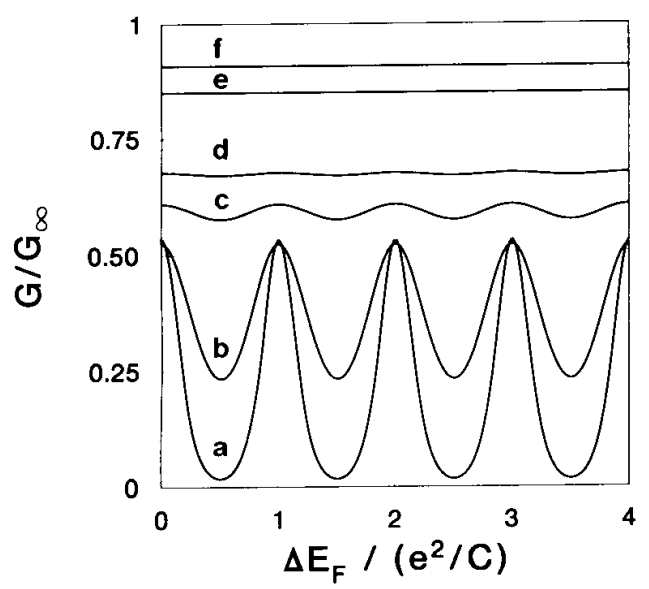

Figure 1.4. Calculated temperature dependence of the Coulomb oscillations as a function of Fermi energy in the classical regime. The parameters are $\Delta E=0.01 e^{2} / C$ and $k_{B} T /\left(e^{2} / C\right)=0.075$ [a], 0.15 [b], 0.3 [c], $0.4[\mathbf{d}], 1$ [e], and 2 [f] [12].

The classical Coulomb blockade regime can be described by the socalled "orthodox" theory $[13,14]$, and the calculated temperature dependence of the Coulomb oscillations is shown in Fig. 1.4a, where the shape of an individual Coulomb peak is described by:

$$
\frac{G}{G_{\infty}}=\frac{1}{2} \cosh ^{-2}\left(\frac{e \cdot\left(C_{\mathrm{g}}-C\right) \cdot\left|V_{\text {gate,res }}-V_{\text {gate }}\right|}{2.5 k_{\mathrm{B}} T}\right)
$$

where $G$ is the conductance through the Coulomb island, $G_{\infty}$ is the conductance trough the barriers at high temperature and $V_{\text {gate,res }}$ is the gate voltage at the resonance. 


\subsection{Charge Transport Through Organic Molecules}

Two most prevalent transport mechanisms through molecular junctions are known and well-studied: coherent non-resonant tunnelling, which dominates in short molecules, and incoherent charge hopping, mostly observed in longconjugated molecular wires [15-17]. In this thesis we focused on molecular self-assembled monolayers (SAMs) of alkenes and $\pi$-conjugated molecules, where transport is generally driven by a non-resonant tunnelling [18].

The molecules can vary in length, structure, packing and bonding group. In this thesis we chose alkanedithiols, oligo(phenylene vinylene) (OPV) and oligo(phenylene ethynylene) (OPE) (Sigma-Aldrich) (Fig. 1.5). Due to a smaller band gap between the highest occupied (HOMO) and lowest unoccupied (LUMO) molecular orbitals (also known as frontier orbitals) $\pi$ conjugated molecules are, in general, better conductors than alkenes [19]. Nevertheless, the local environment and the way the molecules are contacted can resolve whether tunnelling or hopping transport mechanism dominates for OPEs [20].

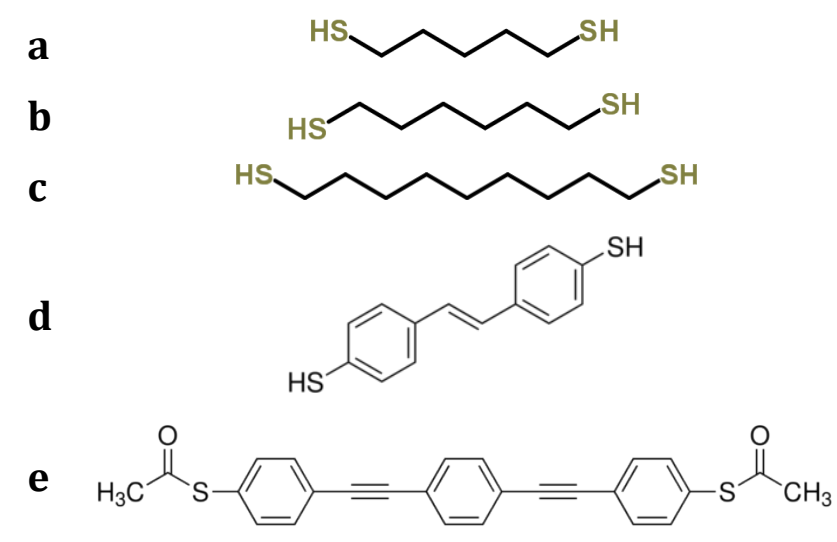

Figure 1.5. Examples of the molecules of interest. a-c, alkanedithiols containing five (a), six (b) and nine (c) carbons; (d), OPV2; (e), OPE3.

Due to a big HOMO-LUMO gap (e.g., 8 eV for alkyl systems) [21] , molecular SAMs embedded between metallic electrodes represent a simple classical molecular junction (Fig. 1.6). Depending on the molecule's characteristics as well as on the metal work function, the distance between Fermi level and closest molecular level can vary. Simons' approximation can 
be used to model the current-voltage behaviour of a molecular junction acting as a tunnel barrier [22].

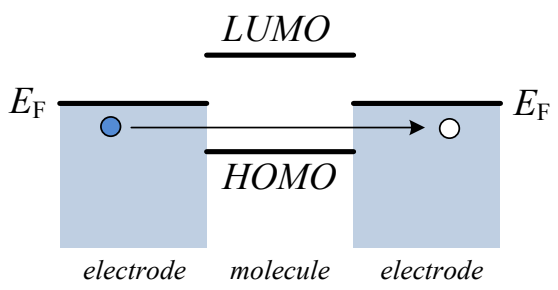

Figure 1.6. Energy band diagram of a molecular junction at zero bias voltage, where $E_{\mathrm{F}}$ is a Fermi energy of the electrodes.

The coherent tunnelling process is temperature-independent and the resistance $(R)$ through the molecular layer exponentially increases with increasing molecular chain length:

$$
R=R_{0} e^{(\beta d)}
$$

where $R_{0}$ is an effective contact resistance that depends on the anchoring group and contact electrodes; and $\beta$ is the tunnelling decay factor that depends on the nature of the molecular structure and applied voltage [16, 23]. More details about the coherent non-resonant tunnelling can be found in Ref. [21].

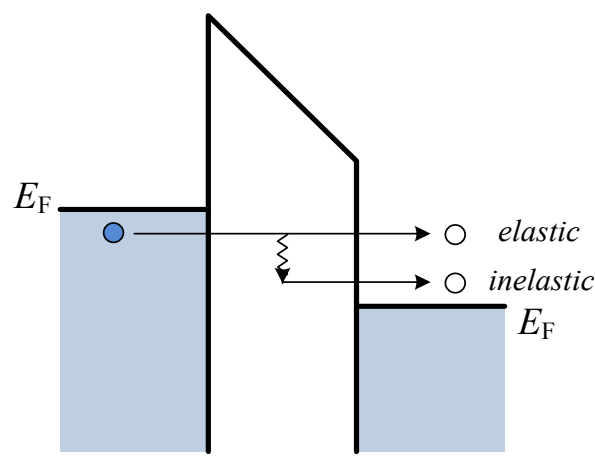

Figure 1.7. Energy band diagram of a molecular tunnelling junction showing elastic and inelastic tunnelling. Inelastic tunnelling is accompanied by the energy loss/gain. $E_{\mathrm{F}}$ is the Fermi energy of the electrodes. 
When under applied bias voltage (Fig. 1.7) an electron tunnels through the molecular junction and its energy is preserved, the process is elastic, thus, coherent tunnelling. Any inelastic event involving energy exchange leads to the incoherent transport through the molecular layer [24].

\subsection{Sample Fabrication and Measurements Techniques}

“Experimental physics cannot do without instruments...”

Jean-Antoine Nollet, 1770

Different techniques and equipment were utilized to fabricate, electrically characterise and analyse all the devices described in this thesis. For the sample fabrication, optical lithography, electron beam lithography (EBL) and electron beam metal evaporation were used. Primary sample characterisation was performed by scanning electron microscopy (SEM) and/or atomic force microscopy (AFM) imaging. Room temperature measurements in high vacuum $\left(\sim 10^{-5}\right.$ mbar $)$ were performed in a probe station followed, if needed, by low temperature measurements in a closed-cycle cryostat or in a cryogen free dilution refrigerator.

Advantages of contact optical lithography are low cost, high throughput and moderately high resolution (limited by the wavelength of light). This technique was utilized to prepare large-area (e.g., $100 \times 100 \mu \mathrm{m}^{2}$ ) electrical contacts where high resolution $(<1 \mu \mathrm{m})$ is not an issue.

EBL is a high-cost technique with low throughput. The main advantage of EBL is a very high resolution. EBL was used only for the fabrication of the most crucial parts of the devices. Sub-20 nm structures were obtained in this thesis using this technique.

\subsubsection{Processing Details}

Four-inch p++ Si (100) (0.010-0.025 $\Omega \cdot \mathrm{cm})$ wafers with a thermally grown 35 $\mathrm{nm} \mathrm{SiO}_{2}$ (Electronics UCL) were used as substrates in all experiments. A highly boron-doped Si substrate underneath the thin oxide layer can be used as a back gate during electrical characterisation.

Prior to the deposition of resists, the wafers were diced into $11 \mathrm{x} 11$ $\mathrm{mm}^{2}$ samples (Dicing saw Disco DAD 321) and then properly cleaned in acetone and isopropanol in a sonication bath followed by the spin-coating of 
polymethylmethacrylate (PMMA 950 A2 or PMMA 950 A4) resist (spun for 1 min at 1500 and $4000 \mathrm{rpm}$ for PMMA $950 \mathrm{~A} 2$ and A4, respectively). Afterwards, samples were baked at $160{ }^{\circ} \mathrm{C}$ for $2 \mathrm{~min}$ using a hot plate or left over night in the wet-bench for degassing.

For the EBL patterning, we employed a Raith 150 TWO (Raith GmbH) system equipped with a thermal field-emission cathode and a laser interferometer stage. The EBL system was operated at $20 \mathrm{kV}$ and with electron beam currents of $36 \mathrm{pA}$ and $1.2 \mathrm{nA}$ for high and low resolution structures, respectively. All structures were exposed in a writing field of $100 \times 100 \mu \mathrm{m}^{2}$ with a step size 2 or $26 \mathrm{~nm}$.

Following the EBL exposure, the resist was developed in a mixture of methylisobutylketone/isopropanol (MIBK/IPA 1:3) for $30 \mathrm{sec}$, the wafers were rinsed in isopropanol and blown dry with nitrogen. Immediately after development, the resist residuals were ashed in $\mathrm{O}_{2}$ plasma for $20 \mathrm{~s}$ at $300 \mathrm{~W}$ with $18 \% \mathrm{O}_{2}$ flow (Tepla 300E $\mathrm{O}_{2}$ plasma etcher) or removed by ozone cleaning for $5 \mathrm{~min}$ (Ozone UV PRS 100 reactor). Following the cleaning procedure, a metal film (Au or $\mathrm{Ti} / \mathrm{Au}$ ) was evaporated in the metal evaporator (BAK 600) with an evaporation rate $\geq 2 \AA /$ s and lifted-off in acetone.

A mask aligner EVG 620 with robot was employed for the optical lithography patterning. Prior to the exposure, primer hexamethyldisilazane (HMDS) was spin-coated for $30 \mathrm{~s}$ at $4000 \mathrm{rpm}$ followed by deposition of the photoresist 907/17 for $30 \mathrm{sec}$ at $4000 \mathrm{rpm}$. After prebaking for $1 \mathrm{~min}$ at 95o samples were exposed for $4 \mathrm{~s}$. Following the exposure, the samples were developed in OPD 4262 (an aqueous solution of tetramethylammonium hydroxide (TMAH) at $2.5 \%$ ) for $60 \mathrm{~s}$, rinsed in deionized water and spun dry with nitrogen blow. After the resist residuals removal and metal film evaporation, it was lifted-off in acetone.

A SEM FEI Sirion HR-SEM, a FEI Focused Ion Beam System (FIB) with a dual beam or Zeiss MERLIN HR-SEM were used for the detailed surface studies of the fabricated samples. Typically, the accelerating voltage of the SEM was $5 \mathrm{kV}$, and the working distance was varying from 6 to $10 \mathrm{~mm}$.

For the AFM imaging, a Bruker ICON was employed when it was crucial to avoid any contamination (especially by carbon deposition due to the SEM imaging). The Bruker ICON AFM also allows to perform automatic imaging.

Primary electrical characterisation of the fabricated devices was performed in a probe station (Janis ST-500) with a two-wire configuration using Beep- $\mathrm{R}$, a source-meter that can quickly probe the resistance by applying a maximum voltage drop of $10 \mathrm{mV}$, and/or Keithley 2400 
SourceMeter controlled by a homemade LabView based program with an a typical applied voltage of $10 \mathrm{mV}$ (up to $1 \mathrm{~V}$ was applied to study electron transport through molecular junctions). The probe station Janis ST-500 allows us to perform measurements in vacuum $\left(\sim 10^{-5} \mathrm{mbar}\right)$, and between $4 \mathrm{~K}$ and $475 \mathrm{~K}$.

Low-temperature and magnetoresistance measurements were carried out in a closed-cycle cryostat (Oxford Instruments Heliox VL) with a base temperature of $230 \mathrm{mK}$ (maximum hold time of 70 hours and a superconducting magnet with a maximum field of $\pm 8 \mathrm{~T}$ ) or in a cryogen free dilution refrigerator (Oxford Instruments Triton ${ }^{\mathrm{TM}} 200$ ) with a base temperature of $7 \mathrm{mK}$ and a vector magnet that can reach $\pm 6 \mathrm{~T}$ in the $z$ direction and $\pm 1 \mathrm{~T}$ in $x$ - and $y$-directions.

In order to improve signal-to-noise ratio a lock-in amplifier (Stanford Research Systems SR830) in combination with IVVI-DAC rack (Quantum Transport designed instrumentation, TU Delft, design by Ing. Raymond Schouten).

In the following experiments we used homocysteine (>95\%, SigmaAldrich), cetyltrimethylammonium bromide (CTAB) (99\%, Aldrich), Au NRs (Nanopartz, OD=1). MiliQ water was used for all solution preparations and experiments.

\subsubsection{Deposition of Self-Assembled Monolayers}

In our experiments we fabricated different devices of a diverse geometry and performance (such as AB rings, SETs based on Au NRs and NPs) which have been functionalised by self-assembled monolayers (SAMs). Functionalisation was performed by immersing samples into a dilute solution of desired molecules. Typical concentration was $5 \mathrm{mM}$ [25]. Alkeindithiols were dissolved in ethanol, while THF (tetrahydrofuran) was used for OPV2 and OPE3 molecules. Samples were ready for further experimentation after 24-48 hours and afterwards cleaned with solvent only and blown dry with nitrogen.

\subsection{Electrical Contacting of Nano Objects by Electron Beam Lithography}

\subsubsection{Bitmap Markers Formation}

When nano objects, randomly placed on the substrate, have to be electrically contacted by EBL, it is crucial to determine the exact location of the nano objects of interest (Chapter 4). In this work we used a $1.5 \times 1.5 \mathrm{~mm}^{2}$ area in the 
middle of the $11 \times 11 \mathrm{~mm}^{2} \mathrm{Si} / \mathrm{SiO}_{2}$ samples to pre-pattern the substrate with a bitmap and four global marks to perform rough alignment. This $1.5 \times 1.5 \mathrm{~mm}^{2}$ space area was divided into $100 \times 100 \mathrm{~mm}^{2}$ writing fields and numbered from A to 0 in the $x$-direction and from 1 to 15 in the $y$-direction (Fig. 1.8a). Every $100 \times 100 \mu \mathrm{m}^{2}$ field (Fig. 1.8b) contains four local alignment markers, which are simple crosses (Fig. 1.8c), and hundred bitmap markers in the middle of the writing field. Each bitmap marker is a unique combination of $100 \times 100$ $\mathrm{nm}^{2}$ squares (e.g. in Fig. 1.8d) with a separation between two neighbouring bitmap markers of $4 \mu \mathrm{m}$.

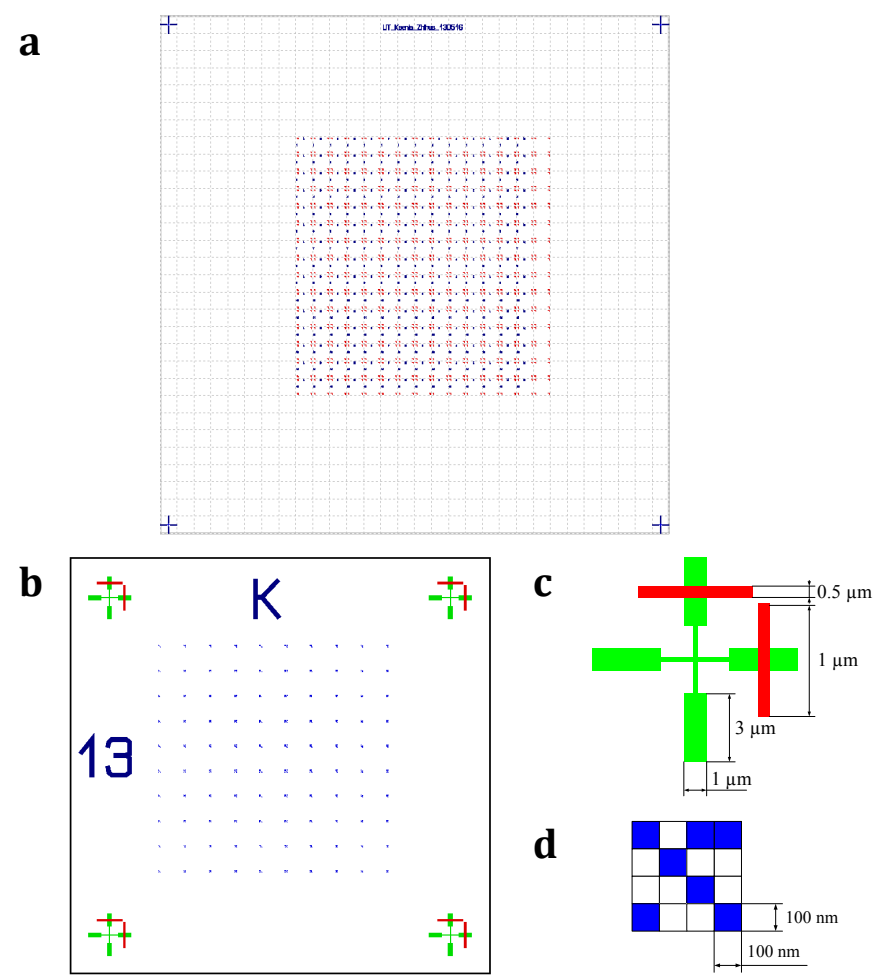

Figure 1.8. EBL design of the pre-patterned substrate. $\mathbf{b}, 100 \times 100 \mu \mathrm{m}^{2}$ writing field, containing alignment (green, c) and bitmap (blue, d) markers, where red areas (c) are used for the automatic alignment between different pattern layers.

The metal thickness of the bitmap and alignment markers has to be chosen such that it gives a good contrast during imaging (when we find the location of an interesting nano object and perform the alignment) and at the 
same time should not be too thick which can give problems during the deposition of contacting electrodes. In our experiments we chose a $3 / 30 \mathrm{~nm}$ $\mathrm{Ti} / \mathrm{Au}$ double metal layer. Figure 1.9 shows a SEM image demonstrating a part of the bitmap with a zoom-in of one of the markers (in the inset). Since every bitmap marker is unique within one writing field we can determine the location of a nano object of interest with a precision limited by atomic force microscopy imaging.

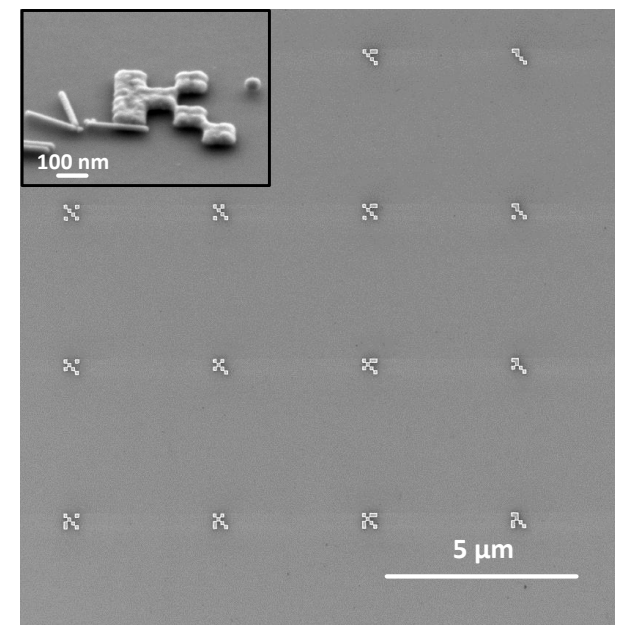

Figure 1.9. $\mathrm{SEM}$ image of a $\mathrm{Ti} / \mathrm{Au}$ bitmap markers on top of $\mathrm{Si} / \mathrm{SiO}_{2}$ substrate used for the identification of NR-NP-NR assemblies deposited afterwards (inset shows a zoom-in of one of the markers).

\subsubsection{Top-Down Electrical Contacting}

Following bottom-up formation of NR-NP-NR assemblies (Chapter 4) we fabricated electrical contacts by EBL with a precision better than $20 \mathrm{~nm}$. Our experiments have shown that prebaking of the resist resulted in a formation of short contacts between NRs and NPs. To avoid this, samples were left over night for the degassing on a wet bench protected on top by the glass beaker and with a nitrogen flow access. Afterwards, an EBL procedure was performed.

Another crucial step is SEM imaging. We observed formation of a thick contamination layer in exposed areas. Figure 1.10a shows AFM image of the contacted NRs-MPs-NRs assembly which has been SEM imaged before (Fig. 1.10b). Scanning with electron beam formed a contamination layer of about 5 
nm (Fig. 1.10c). This dramatically influences the performance of the fabricated devices. Particularly, the formed contamination layer between assemblies and electrical contacts is insulating and results in open circuit.

a

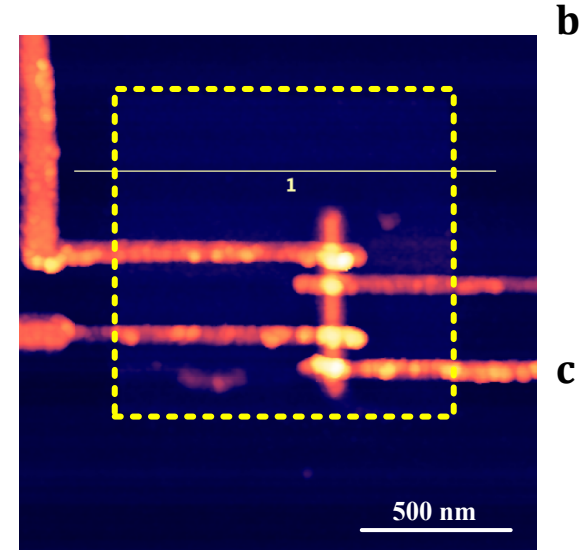

b

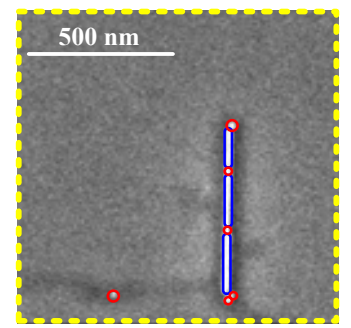

C

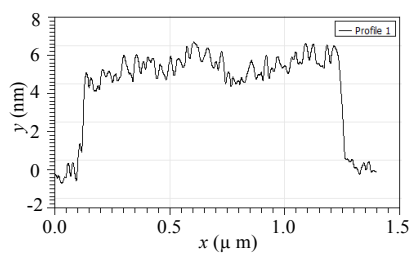

Figure 1.10. a, AFM image of the contacted self-assembled NR-NP structure (defined by blue and red, respectively, in b) after SEM imaging of the high-lighted by yellow dashed line area (b) showing about $5 \mathrm{~nm}$ thick carbon layer (c).

\subsection{Design of Aharonov-Bohm Interferometers}

Quantum-mechanical phenomena like $\mathrm{AB}$ effect can be observed when electrons preserve their phase, i.e. $l_{\mathrm{e}}<l_{\varphi} \sim L$ (where $l_{\mathrm{e}}$ is the elastic scattering length, $l_{\varphi}$ is the phase coherence or inelastic scattering length, and $L$ is the system size, more specifically the length of the circumference of the $A B$ ring) [26]. This condition can be achieved by performing the measurements at low temperature $(\sim 1 \mathrm{~K})$.

In order to distinguish the $\mathrm{AB}$ effect from UCF the aspect ratio between $S_{\text {in }}$ and $S_{\text {arm }}$ should be $\gg 1$ (Section 1.2). As a result, an AB ring should be carefully designed. Figure 1.11 shows a typical $A B$ interferometer studied in this thesis. 


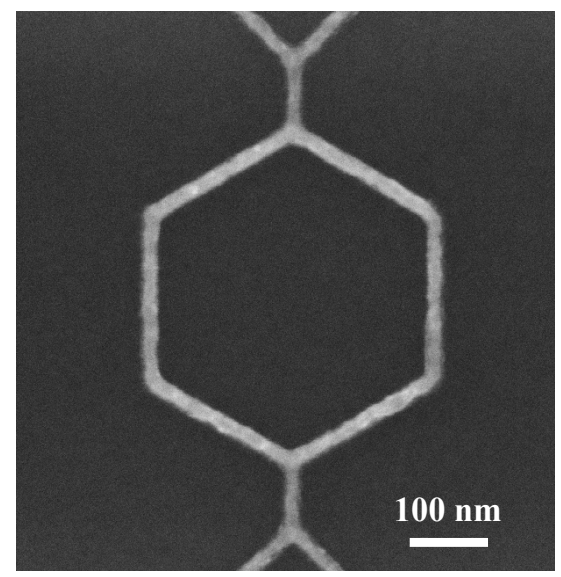

Figure 1.11. SEM image of a typical $A u A B$ interferometer studied in this thesis with a diameter of $500 \mathrm{~nm}$ and arms' width of $20 \mathrm{~nm}$.

\subsection{Low Noise Measurements}

The Coulomb blockade effect can be observed when the charging energy (the energy that is required to charge the Coulomb island with one elementary charge) is larger than the thermal energy of an electron. It can often be achieved by performing charge transport measurements at low temperatures. Depending on the size of the Coulomb island, the effect can generally be observed below liquid nitrogen temperature [27].

In order to observe quantum coherent effects, like the $\mathrm{AB}$ effect, the coherent length $l_{\varphi}$ should be long enough $\left(l_{\varphi} \sim L\right)$ [28]. Therefore, it is necessary to cool down devices to milliKelvin temperatures $(<1 \mathrm{~K})$ where phonon excitations are minimized [29]. Thereby, the thermal energy at certain temperature $T$ limits the excitation current/applied voltage $\left(I \sim e V \ll 3.5 k_{\mathrm{B}} T\right)$ [30], and examples are given in Table 1.1. Since the amplitude of the $A B$ oscillations is generally in order of $\cong 1 \%$ of the main signal $(\Delta I(\Delta V) \cong$ $0.01 I(V)$ ), noise and interference reduction in electrical measurement setup becomes crucial and is somewhat of an art.

Another factor limiting the resolution of the electric signal measured across molecular junctions is high resistance of devices $(I=V / R)$. The resistance of molecular junctions studied in this work and embedded in hybrid inorganic-organic $\mathrm{AB}$ interferometers varies between hundreds of kiloOhms and hundreds of GigaOhms. Nevertheless, the resolution of the measurements of the sample resistance $R$ is limited by the cut-off frequency $f_{\mathrm{c}}$ 
due to the capacitances $C$ (which are in order of nanoFarad) in filters in the measurement system:

$$
\left.f_{c}=\frac{1}{2 \pi R C} \rightarrow R_{\max }=\frac{1}{2 \pi f_{\mathrm{c} C} C} \approx \frac{0.2 \cdot 10^{9}}{f_{c}}=2 \mathrm{M} \Omega \text {, (for } f_{\mathrm{c}}=100 \mathrm{~Hz}\right)
$$

Noise is caused by a large number of processes; each of them has very small magnitudes. Johnson noise, shot noise and $1 / f$ noise contribute the overall noise level. Johnson noise (also known as white noise, thermal noise or

Table 1.1. Calculated maximum applied voltage $V$ across the $A B$ interferometer and maximum allowed current $I$ along the device for a quantum resistance $R_{0}=h / e^{2}=26 \mathrm{k} \Omega$ and for $R_{\max }=2 \mathrm{M} \Omega$ at different temperatures $T$.

\begin{tabular}{c|llll}
\hline$T$ & & \multicolumn{1}{c}{$V$} & \multicolumn{1}{c}{$I\left(R_{0}\right)$} & $I\left(R_{\max }\right)$ \\
\hline \multirow{2}{*}{$10 \mathrm{mK}$} & $\mathrm{k}_{\mathrm{B}} \mathrm{T}$ & $0.86 \mu \mathrm{V}$ & $33.3 \mathrm{pA}$ & $0.43 \mathrm{pA}$ \\
& $3.5 \mathrm{k}_{\mathrm{B}} \mathrm{T}$ & $3.01 \mu \mathrm{V}$ & $116.5 \mathrm{pA}$ & $1.51 \mathrm{pA}$ \\
$50 \mathrm{mK}$ & $\mathrm{k}_{\mathrm{B}} \mathrm{T}$ & $4.3 \mu \mathrm{V}$ & $166.5 \mathrm{pA}$ & $2.15 \mathrm{pA}$ \\
& $3.5 \mathrm{k}_{\mathrm{B}} \mathrm{T}$ & $15.1 \mu \mathrm{V}$ & $0.58 \mathrm{nA}$ & $7.53 \mathrm{pA}$ \\
& $\mathrm{k}_{\mathrm{B}} \mathrm{T}$ & $8.6 \mu \mathrm{V}$ & $0.33 \mathrm{nA}$ & $4.3 \mathrm{pA}$ \\
& $3.5 \mathrm{k}_{\mathrm{B}} \mathrm{T}$ & $30.1 \mu \mathrm{V}$ & $1.17 \mathrm{nA}$ & $15.1 \mathrm{pA}$ \\
$300 \mathrm{mK}$ & $\mathrm{k}_{\mathrm{B}} \mathrm{T}$ & $25.8 \mu \mathrm{V}$ & $0.99 \mathrm{nA}$ & $12.9 \mathrm{pA}$ \\
& $3.5 \mathrm{k}_{\mathrm{B}} \mathrm{T}$ & $90.1 \mu \mathrm{V}$ & $3.49 \mathrm{nA}$ & $45.15 \mathrm{pA}$ \\
$500 \mathrm{mK}$ & $\mathrm{k}_{\mathrm{B}} \mathrm{T}$ & $43 \mu \mathrm{V}$ & $1.67 \mathrm{nA}$ & $21.5 \mathrm{pA}$ \\
& $3.5 \mathrm{k}_{\mathrm{B}} \mathrm{T}$ & $150 \mu \mathrm{V}$ & $5.8 \mathrm{nA}$ & $75.3 \mathrm{pA}$ \\
$1 \mathrm{~K}$ & $\mathrm{k}_{\mathrm{B}} \mathrm{T}$ & $86 \mu \mathrm{V}$ & $3.3 \mathrm{nA}$ & $43 \mathrm{pA}$ \\
& $3.5 \mathrm{k}_{\mathrm{B}} \mathrm{T}$ & $301 \mu \mathrm{V}$ & $11.7 \mathrm{nA}$ & $151 \mathrm{pA}$ \\
\hline
\end{tabular}

Nyquist noise), generated by a resistor, depends on the temperature and the bandwidth. Shot noise generated by the quantized nature of charge is proportional to the bias current and the bandwidth. Noise generated in electronics is $1 / f$ noise (also called flicker noise) which follows 1 /frequency curve. The origin of the $1 / f$ is not well-known. It may be explained by slow variations of the resistance given slow voltage fluctuations under constant current, by charging and discharging of an impurity (jumping of the conductance between more stable values), by technological processes $(1 / f$ noise is more likely to appear close to metal-insulator transition), and so on [31]. Very often noise can be decreased by averaging over many measurements. Unfortunately, $1 / f$ noise cannot be improved by averaging, it is a so-called "random walk" [31].

Typical working frequencies were 17.77 and $13.33 \mathrm{~Hz}$. S4c current/voltage and S3b voltage/current source modules (of the IVVI-DAC rack) were employed to amplify the excitation signal, while measurement modules M2d voltage-measure and M1b current-measure were used to detect the signal. Model M2d has $0.8 \mathrm{nV} / \sqrt{\mathrm{Hz}}$ input noise voltage and model M1b has a noise floor down to $5 \mathrm{fA} / \sqrt{\mathrm{Hz}}$. 
Apart from the noise, the interference is another kind of the disturbance in the measurement setup. To prevent interference in the system a few actions have to be taken: to prevent $50 \mathrm{~Hz}$ interference all elements directly connected to the sample are purely analog (no clock generator) and battery driven; the measurement setup consists of multiple twisted pairs in loops; all measurement elements were connected to a single ground and isolated from other possible ground connections by using wooden shelves for the measurement electronics, rubber pumping lines and plastic connectors for the helium recovery lines.

\section{REFERENCES}

[1] A. Hatzor and P. S. Weiss, "Molecular Rulers for Scaling Down Nanostructures," Science, vol. 291, pp. 1019-1020, February 9, 20012001.

[2] K. Likharev, "Electronics Below $10 \mathrm{~nm}, "$ in Nano and Giga Challenges in Microelectronics, J. G. K. Labanowski, Ed., ed Amsterdam: Elsevier Science B.V., 2003, pp. 27-68.

[3] C. Joachim, J. K. Gimzewski, and A. Aviram, "Electronics using hybridmolecular and mono-molecular devices," Nature, vol. 408, pp. 541-548, 11/30/print 2000.

[4] Y. Aharonov and D. Bohm, "Significance of Electromagnetic Potentials in the Quantum Theory," Physical Review, vol. 115, pp. 485-491, 08/01/ 1959.

[5] R. A. Webb, S. Washburn, C. P. Umbach, and R. B. Laibowitz, "Observation of h/e Aharonov-Bohm oscillations in normal-metal rings," Physical Review Letters, vol. 54, pp. 2696-2699, Jun 241985.

[6] (2010). Seven wonders of the quantum world. Available: http://www.newscientist.com/special/sevenwonders-of-the-quantum-world

[7] A. D. Stone, "Magnetoresistance Fluctuations in Mesoscopic Wires and Rings," Physical Review Letters, vol. 54, pp. 2692-2695, 06/24/ 1985.

[8] B. L. Al'tshuler, "Fluctuations in the extrinsic conductivity of disordered conductors," JETP letters, vol. 41, p. 648, 1985.

[9] S. Washburn and R. A. Webb, "Aharonov-Bohm effect in normal metals: quantum coherence and trasnport," Advances in Physics, vol. 35, pp. 375422, Jul-Aug 1986.

[10] C. W. J. Beenakker, "Theory of Coulomb-blockade oscillations in the conductance of a quantum dot," Physical Review B, vol. 44, pp. 16461656, 07/15/ 1991.

[11] L. L. Sohn, L. P. Kouwenhoven, and G. Schön, "Mesascopic electron transport," pp. 105-176, 1997. 
[12] H. van Houten, C. W. J. Beenakker, and A. A. M. Staring, "CoulombBlockade Oscillations in Semiconductor Nanostructures," ed, 2005.

[13] I. O. Kulik and R. I. Shekhter, "Kinetic phenomena and charge discreteness effects in granular media," Soviet Physics JETP, vol. 41, 1975.

[14] D. V. Averin and K. K. Likharev, "Coulomb blockade of single-electron tunneling, and coherent oscillations in small tunnel junctions," Journal of Low Temperature Physics, vol. 62, pp. 345-373, 1986/02/01 1986.

[15] H. Song, M. A. Reed, and T. Lee, "Single Molecule Electronic Devices," Advanced Materials, vol. 23, pp. 1583-1608, 2011.

[16] G. Wang, T.-W. Kim, and T. Lee, "Electrical transport characteristics through molecular layers," Journal of Materials Chemistry, vol. 21, pp. 18117-18136, 2011.

[17] H. B. Akkerman and B. d. Boer, "Electrical conduction through single molecules and self-assembled monolayers," Journal of Physics: Condensed Matter, vol. 20, p. 013001, 2008.

[18] G. C. Solomon, C. Herrmann, T. Hansen, V. Mujica, and M. A. Ratner, "Exploring local currents in molecular junctions," Nature Chemitry, vol. 2, pp. 223-228, 03//print 2010.

[19] B. A. Mantooth and P. S. Weiss, "Fabrication, assembly, and characterization of molecular electronic components," Proceedings of the IEEE, vol. 91, pp. 1785-1802, 2003.

[20] Y. Selzer, L. Cai, M. A. Cabassi, Y. Yao, J. M. Tour, T. S. Mayer, et al., "Effect of Local Environment on Molecular Conduction: Isolated Molecule versus Self-Assembled Monolayer," Nano Letters, vol. 5, pp. 6165, 2005/01/01 2004.

[21] J. M. Beebe, B. Kim, J. W. Gadzuk, C. Daniel Frisbie, and J. G. Kushmerick, "Transition from Direct Tunneling to Field Emission in Metal-Molecule-Metal Junctions," Physical Review Letters, vol. 97, p. $026801,07 / 10 / 2006$.

[22] J. G. Simmons, "Generalized Formula for the Electric Tunnel Effect between Similar Electrodes Separated by a Thin Insulating Film," Journal of Applied Physics, vol. 34, pp. 1793-1803, 1963.

[23] G. Wang, T.-W. Kim, H. Lee, and T. Lee, "Influence of metal-molecule contacts on decay coefficients and specific contact resistances in molecular junctions," Physical Review B, vol. 76, p. 205320, 11/20/ 2007.

[24] C. J. Adkins and W. A. Phillips, "Inelastic electron tunnelling spectroscopy," Journal of Physics C: Solid State Physics, vol. 18, p. 1313, 1985.

[25] J. C. Love, L. A. Estroff, J. K. Kriebel, R. G. Nuzzo, and G. M. Whitesides, "Self-Assembled Monolayers of Thiolates on Metals as a Form of Nanotechnology," Chemical Reviews, vol. 105, pp. 1103-1170, 2005/04/01 2005. 
[26] C. Harmans and F. d. T. N. TU Delft, Mesoscopic Physics: An Introduction : TN385 [dictaat Behorende Bij College TN3853]: TU Delft, 2003.

[27] L. L. Sohn, L. P. Kouwenhoven, and G. Schön, "Mesoscopic electron transport," 1997.

[28] G. Dumpich and A. Carl, "Anomalous temperature dependence of the phase-coherence length for inhomogeneous gold films," Physical Review B, vol. 43, pp. 12074-12077, 05/15/ 1991.

[29] K. Aihara, M. Yamamoto, K. Iwadate, and T. Mizutani, "Temperature Dependence of the Phase Coherence Length of High-Mobility AlGaAs/GaAs Quantum-Wire Rings," Japanese Journal of Applied Physics, vol. 30, p. L1627, 1991.

[30] E. B. Foxman, U. Meirav, P. L. McEuen, M. A. Kastner, O. Klein, P. A. Belk, et al., "Crossover from single-level to multilevel transport in artificial atoms," Physical Review B, vol. 50, pp. 14193-14199, 11/15/ 1994.

[31] P. Horowitz and W. Hill, The Art of Electronics: Cambridge University Press, 1989. 


\section{Chapter 2 NANOPARTICLE-BRIDGED MoleCUlaR JUNCTIONS}

A high yield, reproducible and simple technique for fabrication of nanometer spaced electrodes by dielectrophoresis (DEP) is reported in this chapter. The method was used for the creation of molecular junctions by bridging a nanogap between gold leads, functionalized by a self-assembled organic monolayer (SAM), with gold nanoparticles (Au NPs) in order to study charge transport through self-assembled organic molecular monolayers. We perform electrical characterisation of the fabricated devices to examine transport properties dependent on the molecule's characteristic (e.g. length, chemical composition, chemical bonding) and external parameters (e.g. temperature, electrostatic potential). We show an application of DEP method for fabrication of singleelectron transistors (SETs) and designless Boolean logic devices. 
Research interest in hybrid inorganic-organic electronics is increased in recent years. To be able to build molecule-based devices it is very important to study, understand and control electronic transport at the level of a single or a few molecular junctions. For this purpose building nanometer spaced gaps is necessary to embed single or a few molecules (with a common length in the order of 1-2 nm) in between. Standard top-down lithography technologies do not allow this. There are a few well-known techniques for the creation a single molecule device, such as mechanical break junctions [1], nanopores [2, 3], electromigration [4], shadow evaporation [5], scanning tunnelling microscopy [6]. Unfortunately, they are not very reproducible and require high skills and precision.

In this thesis a simple and very reproducible technique was chosen for the manipulation of single NPs and fabrication of molecular junctions and study charge transport properties of the molecular monolayers. This is the DEP manipulation method, which avoids the requirement for creating extremely small nanogaps $(<1-2 \mathrm{~nm})$ for the direct fabrication of a molecular bridge, and will be described below.

\subsection{Dielectrophoresis Manipulation Method}

DEP is a powerful tool for the controlled manipulation of small polarizable objects (such as metal and semiconducting particles, nanowires, DNA molecules and graphene) by a non-uniform electric field (Fig. 2.1) [7, 8]. This technique, introduced by Pohl [9], is widely used as a method for the creation of molecular junctions by bridging the nanogap between electrodes with NPs with great selectivity. When the electrodes are immersed into a liquid solvent, upon applying electric field the dissolved particles move towards the gap, where the electric field gradient is the largest (Fig. 2.1).
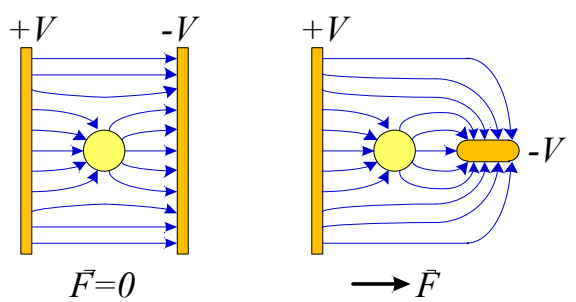

Figure 2.1. Schematic picture of the DEP process. 
The DEP force acting on the particle strongly depends on the permittivities and the conductivities of the medium and the particle, particle's volume and shape, time of the process, applied voltage and frequency. For a spherical particle the DEP force can be expressed as [8]:

$$
\begin{gathered}
F_{\mathrm{DEP}}=2 \pi \varepsilon_{0} \varepsilon_{\mathrm{m}} a^{3} \operatorname{Re}[K(\omega)] \nabla E_{\mathrm{rms}}^{2} \\
\operatorname{Re}[K(\omega)]=\frac{\left(\varepsilon_{\mathrm{p}}-\varepsilon_{\mathrm{m}}\right)\left(\varepsilon_{\mathrm{p}}+2 \varepsilon_{\mathrm{m}}\right)+\frac{1}{\omega^{2}}\left(\sigma_{\mathrm{p}}-\sigma_{\mathrm{m}}\right)\left(\sigma_{\mathrm{p}}+2 \sigma_{\mathrm{m}}\right)}{\left(\varepsilon_{\mathrm{p}}+2 \varepsilon_{\mathrm{m}}\right)^{2}+\frac{1}{\omega^{2}}\left(\sigma_{\mathrm{p}}+2 \sigma_{\mathrm{m}}\right)^{2}},
\end{gathered}
$$

where $\varepsilon_{\mathrm{m}}$ and $\varepsilon_{\mathrm{p}}$ are the permittivities of the medium and the particle, $a$ is the radius of the NPs, $K(\omega)$ is the Clausius-Mossotti factor, $E_{\text {rms }}$ is the root-meansquare value of the electric field, $\sigma_{\mathrm{m}}$ and $\sigma_{\mathrm{p}}$ are the conductivities of the medium and the particle, and $\omega$ is $2 \pi f$.

\subsection{Device Fabrication and Experimental Setup}

Following the fabrication of the nanogaps and formation of the SAM on top of the electrodes, manipulation of the $\mathrm{Au}$ NPs and room temperature characterisation is carried out in a low temperature probe station. A sample is placed in the probe station and a colloidal solution of Au NPs is drop-casted on the sample's surface. Afterwards AC (alternating) or DC (direct) voltage is applied across probe needles placed on top of the electrodes. A series resistor is tuned to prevent high current flow that could break the nanoscopic metallic electrodes, schematic image of the setup is depicted in Fig. 2.2.

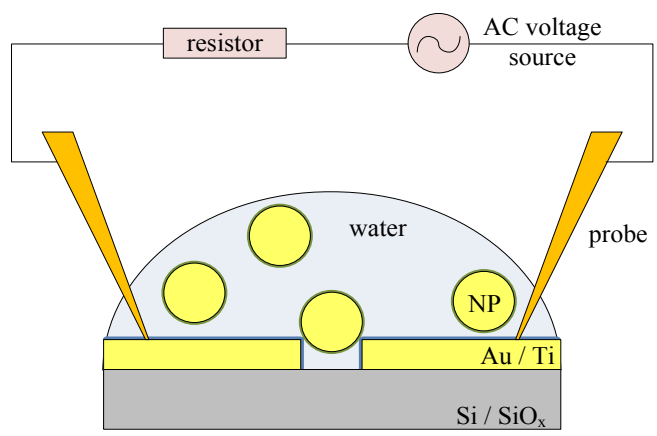

Figure 2.2. Schematic of the setup for the DEP trapping experiment. Cross-section of a nanogap, functionalized by the SAM and bridged with Au NP forming a metal-molecule-metal-molecule-metal contact. 
The Clausius-Mossotti factor (Eq. 2.2) highly depends on the frequency of the applied voltage causing positive or negative DEP [10]. In this work AC voltage (generated by Stanford DS345 Function Generator) is set at $1 \mathrm{MHz}$ and the amplitude of the signal is controlled by a custom-made LabView-based program. By varying Au NPs size (compare to the dimensions of the nanogap) and concentration in a solution, trapping time and voltage, it is possible to limit the amount of particles trapped between electrodes.

a

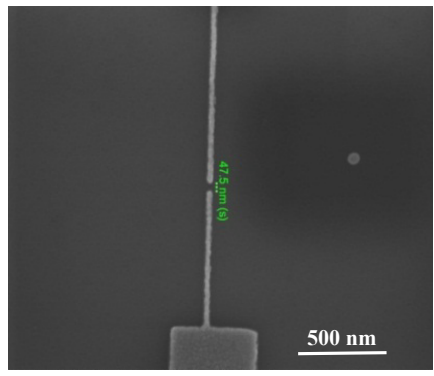

C

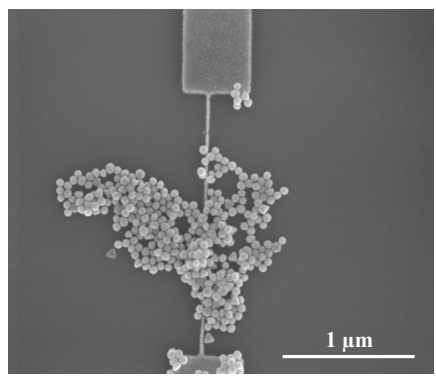

b

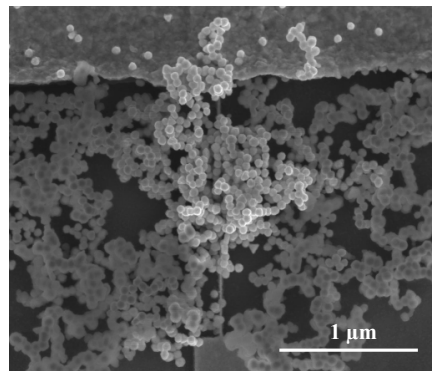

d

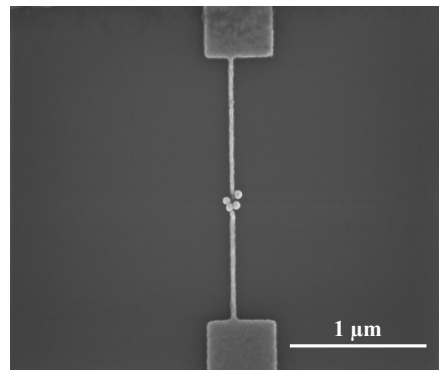

Figure 2.3. Dependence of the DEP process on the NPs' concentration. SEM images of $60 \mathrm{~nm}$ Au NPs trapped in a $50 \mathrm{~nm}$ nanogap (depicted in a) for different concentrations of NPs: $\mathbf{b}$, original solution; $\mathbf{c}, 1: 4$ diluted in water; $\mathbf{d}, 1 ; 10$ diluted in water. Trapping time is $10 \mathrm{sec}, \mathrm{AC}$ voltage is $1 \mathrm{~V}_{\mathrm{pp}}$ at $1 \mathrm{MHz}$, series resistance is $10 \mathrm{M} \Omega$.

\subsection{Concentration, Time and Voltage Dependences}

In order to control the DEP procedure, trapping parameters have to be carefully adjusted. Two of the most important of them are the size and the concentration of the NPs in a medium. In this chapter commercially available citrade-functionalized 20,60 and $100 \mathrm{~nm}$ in diameter Au NPs (British Biocell International) diluted in water with concentrations of $7.00 \times 10^{11}, 2.60 \times 10^{10}$ and $5.60 \times 10^{9}$ particles $/ \mathrm{ml}$, respectively, are tested in all the experiments. As 
shown in Fig. 2.3b, when the original solution of $60 \mathrm{~nm} \mathrm{Au} \mathrm{NPs} \mathrm{is} \mathrm{used} \mathrm{a} \mathrm{NPs'}$ cluster forms around the $50 \mathrm{~nm}$ nanogap (Fig. 2.3a). Manipulation of one or a few NPs is possible after dilution of one part of the commercial solution with ten parts of the Milli-Q water (Fig. 2.3d).

When the NPs' concentration is adjusted, Au nanorings (such geometry was used for the fabrication of hybrid inorganic-organic AharonovBohm (AB) interferometers, see chapter 7) containing two nanogaps in parallel are used to test the trapping time and applied voltage. Figure 2.4 shows that both long trapping time and high peak-to-peak voltage lead to a formation of NPs' cluster around nanogaps and appearing of the electromigration in the narrow interconnecting lines between the ring and contacting leads, causing formation of new nanogaps that are filled by NPs. A series resistor is introduced and tuned to $1 \mathrm{M} \Omega$ to limit the maximum current allowed in the system.

The analysis of all the devices shows that manipulation of an exact amount of NPs is very difficult. Nevertheless, controlling the trapping parameters allows to trap a number of NPs with a certain order of magnitude. In figures 2.5-2.6 there are examples of one and double parallel $50 \mathrm{~nm}$ nanogaps bridged by a single $60 \mathrm{~nm}$ Au NPs.

a

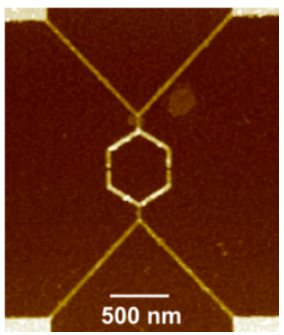

d

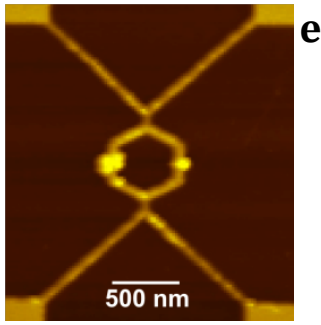

b
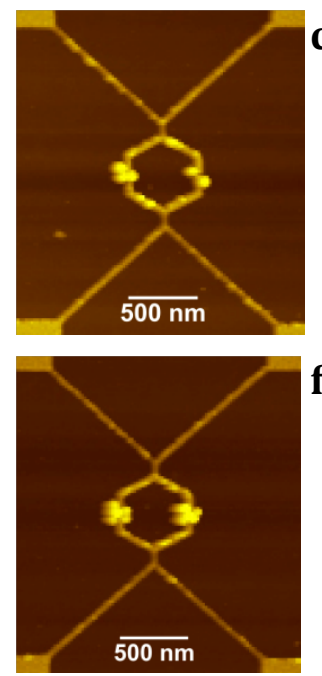
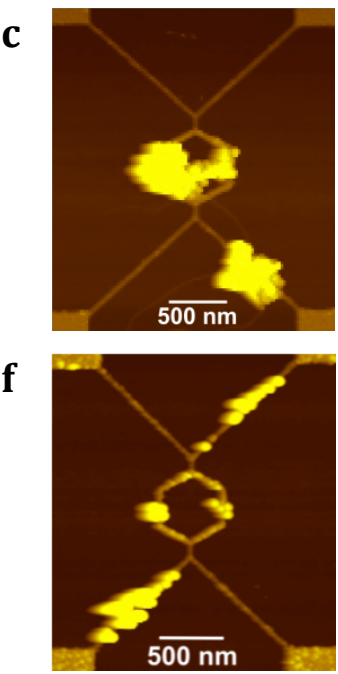

Figure 2.4. AFM images of $60 \mathrm{~nm}$ Au NP trapped in and around $40 \mathrm{~nm}$ nanogap embedded in the arms of $A u A B$ ring. a-c, applied voltage is 1.5 $V$ at $1 \mathrm{MHz}$, series resistance is $1 \mathrm{M} \Omega$, trapping time is (a) $5 \mathrm{sec}$ (b) 10 sec and (c) $15 \mathrm{sec}$. d-f, trapping time is $5 \mathrm{sec}$, series resistance is $1 \mathrm{M} \Omega$, applied voltage is (d) $1.7 \mathrm{~V}$, (e) $1.9 \mathrm{~V}$ and (f) $2.2 \mathrm{~V}$ at $1 \mathrm{MHz}$. 


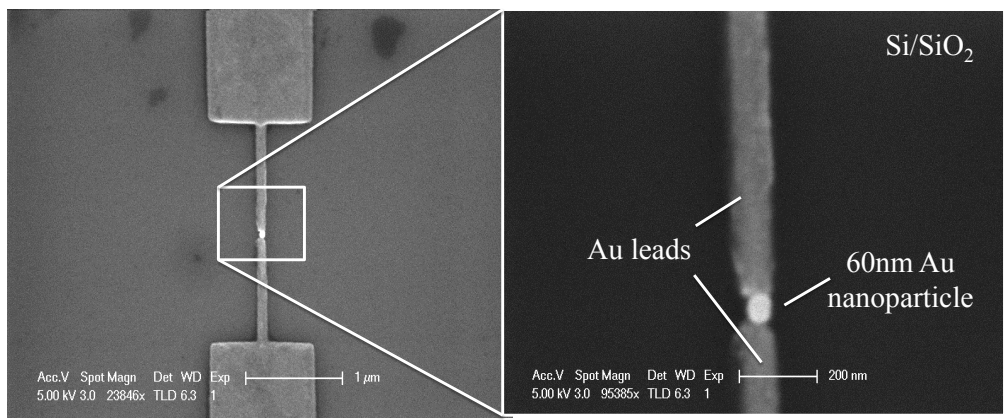

Figure 2.5. SEM image of a single $60 \mathrm{~nm}$ Au NP trapped in a $40 \mathrm{~nm}$ nanogap. Trapping time is $5 \mathrm{sec}$, applied voltage $1.5 \mathrm{~V}$ at $1 \mathrm{MHz}$, series resistance is $1 \mathrm{M} \Omega$.

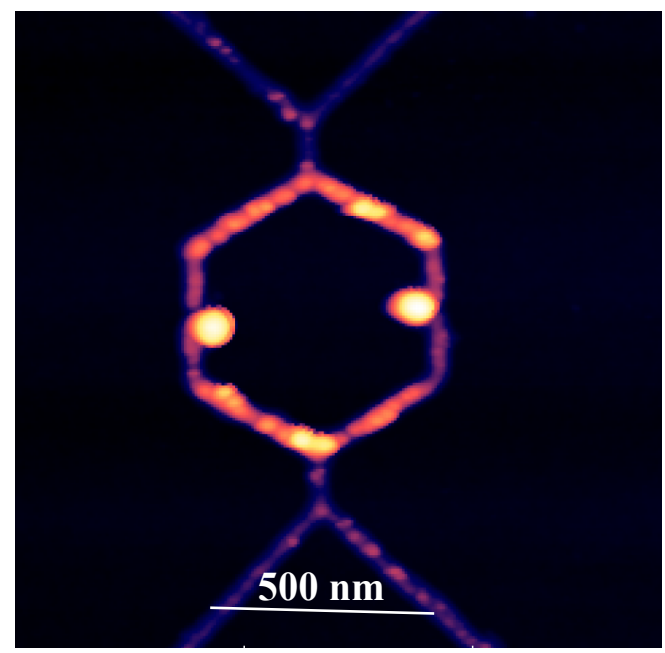

Figure 2.6. AFM image of single $60 \mathrm{~nm}$ Au NP trapped in each $40 \mathrm{~nm}$ nanogap embedded in the arms of $A u A B$ ring. Trapping time is $5 \mathrm{sec}$, applied voltage $1.7 \mathrm{~V}$ at $1 \mathrm{MHz}$, series resistance is $1 \mathrm{M} \Omega$.

\subsection{Current-Voltage Measurements through Molecular Monolayers in Nanoparticle Bridges}

Preliminary characterisation of the charge transport through molecular SAMs in NPs bridges is performed in vacuum (below 10-4 mbar) and at room temperature. Current-voltage $(I-V)$ measurements are carried out in a probe station in a two-probe configuration using a Keithley 2400 SourceMeter and 
controlled via a custom-made LabView-based program. Figure 2.7 shows a representative $I-V$ characteristic for one of the devices (100 nm Au NP bridge junction coated by 1,5-pentanedithiol). The non-linear behaviour in Fig. 2.7 is typical of coherent non-resonant charge tunnelling, which has been widely observed for charge transport through molecular junctions [11-13] (Section 1.4).

Devices showing such non-linear behaviour are taken into account as "working". When a short is measured or the signal is in the noise level, the devices are considered as "non-working" and their data is excluded from this thesis. The yield of the "working devices" is about $50 \%$.

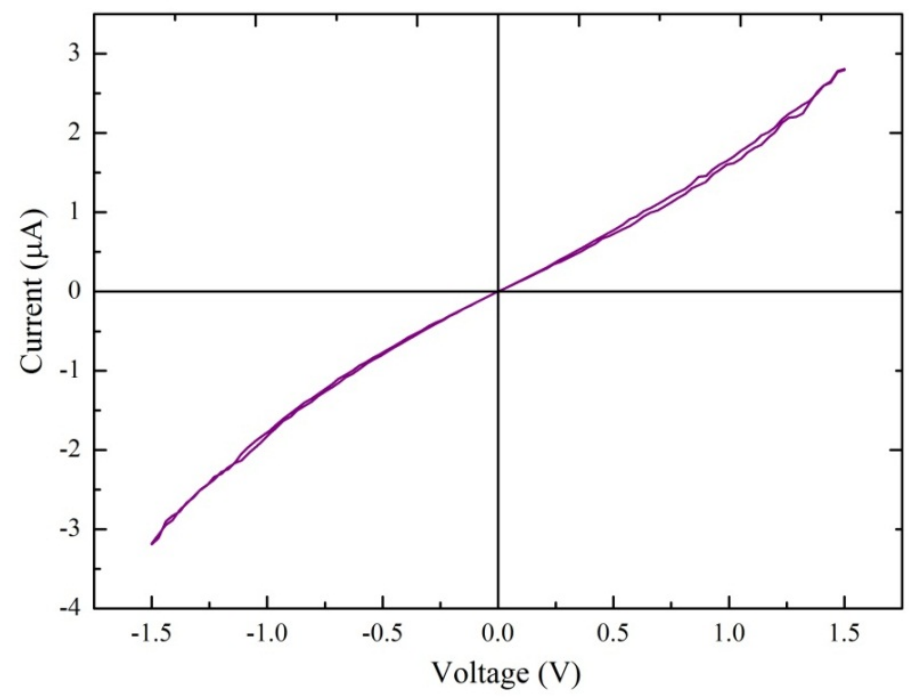

Figure 2.7. $I-V$ characteristic of a junction coated by 1,5-pentanedithiol and bridged by $100 \mathrm{~nm}$ Au NP showing the non-resonant tunnelling behaviour. $\mathrm{T}=295 \mathrm{~K}$.

The examination of characteristic temperature, length, structure and contact dependences on the charge transport is necessary for the reason that it can reveal the charge transport mechanism through molecular SAMs. Figure 2.8 shows statistical histograms of $\log _{10}[R(\Omega)]$ ( $R$ is the resistance of the junction), measured at $0.1 \mathrm{~V}$ for the junctions bridged by $60 \mathrm{~nm}$ Au NPs and functionalized by 1,5-pentanedithiol (Fig. 2.8, green), 1,6-hexanedithiol (Fig. 2.8, red) and 1,9-nonanedithiol (Fig. 2.8, blue), representing the length 
dependence through the dithiol molecules. Such a statistical analysis based on a large amount of measurements data is of high importance due to the reliability issues of molecular junctions $[7,13,14]$.

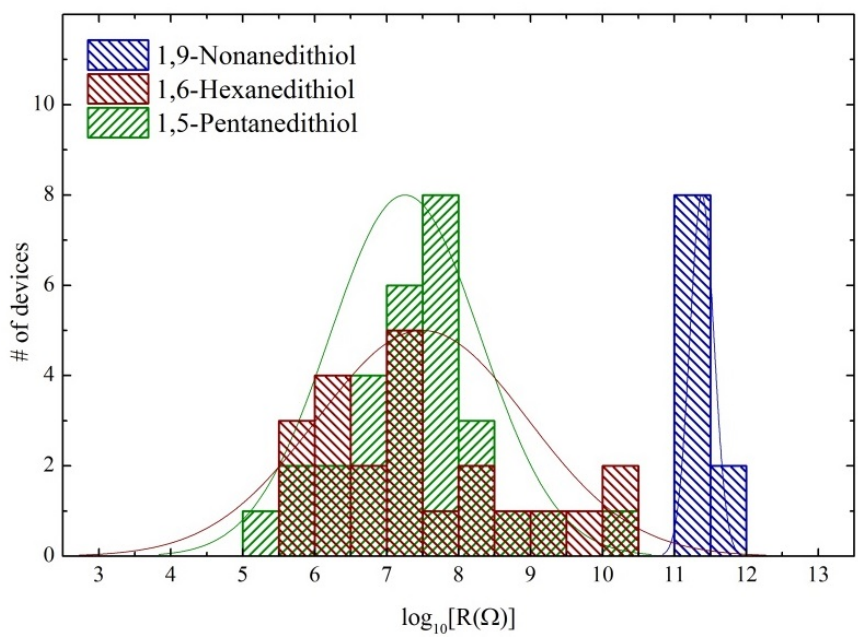

Figure 2.8. Statistical histograms of $\log _{10}[R(\Omega)]$ measured at $0.1 \mathrm{~V}$ for 40 $\mathrm{nm}$ Au junctions bridged by $60 \mathrm{~nm}$ Au NPs and functionalized by 1,5pentanedithiol (green), 1,6-hexanedithiol (red) and 1,9-nonanedithiol (blue).

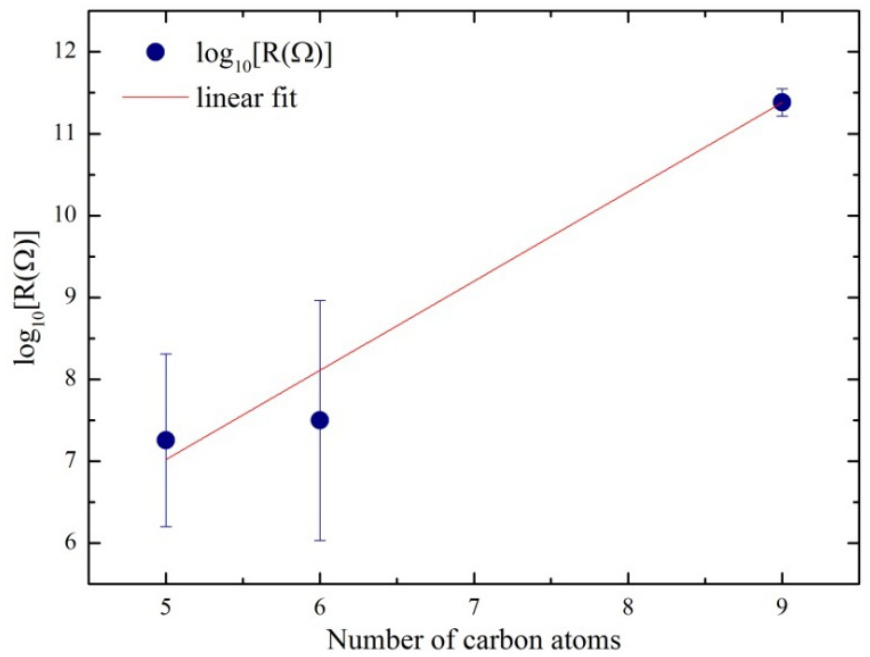

Figure 2.9. Semilog plot of $\log _{10}[R(\Omega)]$ versus the chain length of the alkanedithiols. The extracted decay factor is $1.1 \pm 0.1 \AA^{-1}$. 
Transport through alkanedithiols is in general a coherent tunnelling which strongly depends on the effective contact resistance and the tunnelling decay factor $\beta$ (Eq. 1.8) (Section 1.4). The semilog plot in fig. 2.9 represents a clear exponential dependence of the resistance on the length of the alkanedithiol chain, based on the data represented in fig. 2.8. The decay factor extracted from the linear fit is $1.1 \pm 0.1 \AA^{-1}$ and in good agreement with the literature values which lie between 0.75 and $1.15 \AA^{-1}[13,15]$. Thuo and Reus, et al [14] demonstrated a difference in rates of charge transport through molecular SAMs with odd- and even-numbered $n$-alkanedithiols. This fact may explain the lowered position of the data point for 1, 6-hexanedithiol with respect to the linear fit in fig. 2.9.

Electrical measurements at different temperatures show, in accordance with the theory and literature, that the electron transport through alkanedithiols weakly depends on the external parameters such a temperature and magnetic field. (Measurement data is not included in this thesis). The influence of the anchoring group is not studied in present work.

Figure 2.10 represents statistical histograms of $\log _{10}[R(\Omega)]$ measured at $0.1 \mathrm{~V}$ for the junctions bridged by $60 \mathrm{~nm}$ Au NPs and functionalized by 4,4'dimercaptostilbene (OPV2) and S,S'-[1,4-Phenylenebis(2,1-ethynediyl-4,1phenylene)]bis(thioacetate) (OPE3), and based on the data from "working" devices. As expected [16, 17], OPV2 and OPE3 are better conductors than alkanes, and OPV2 molecules show much lower resistance compare to OPE3.

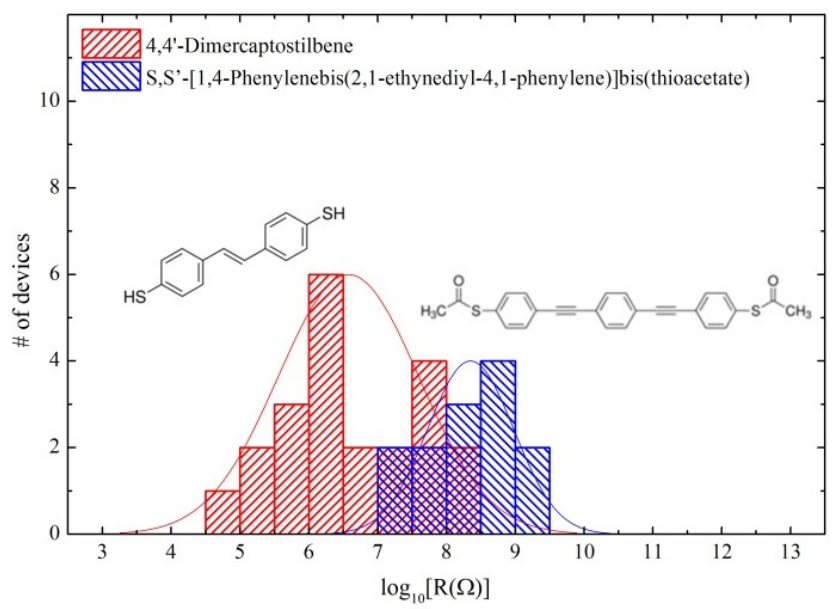

Figure 2.10. Statistical histograms of $\log _{10}[R(\Omega)]$ measured at $0.1 \mathrm{~V}$ for 40 $\mathrm{nm}$ Au junctions bridged by $60 \mathrm{~nm}$ Au NPs and functionalized by $4,4^{\prime}$ dimercaptostilbene (red) and S,S'-[1,4-Phenylenebis(2,1-ethynediyl-4,1phenylene)]bis(thioacetate) (blue). 


\subsection{Molecular Exchange}

An additional advantage of the DEP is that molecular exchange can be performed without changing the device structure. Molecular exchange is a chemical process able to replace the molecules of a SAM monolayer by simply immersing the device in a different molecular solution. If the concentration of the new molecules and the strength of the new chemical bounds is high enough, the new molecules will replace the old ones forming a new SAM. Bernard et al. [18] demonstrated successful molecular exchange between the conjugated oligomers and alkanethiol SAMs with a NPs array network. Molecular exchange in a $60 \mathrm{~nm}$ Au NPs bridged molecular junction is depicted in Fig. 2.11. Observed change in the resistance with every exchange step is in agreement with the previously obtained results (Section 1.4).
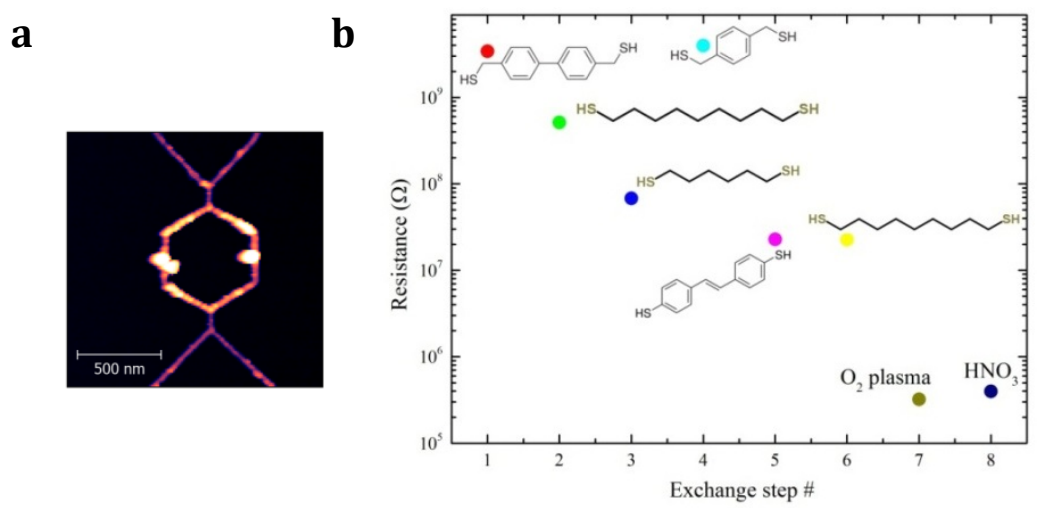

Figure 2.11. a, AFM image of the ring containing $40 \mathrm{~nm}$ junctions and bridges by $60 \mathrm{~nm}$ in diameter Au NPs. b, Dependence of the resistance through the metal-molecule-NPs-molecule-metal parallel junctions (depicted in a) on the molecule for different exchange steps: (1) 4,4'Bis(mercaptomethyl)biphenyl; (2) 1,9-Nonanedithiol; (3) 1,6Hexanedithiol; (4) 1,4-Benzenedimethanethiol; (5) 4,4'Dimercaptostilbene; (6) 1,9-Nonanedithiol; (7) after $\mathrm{O}_{2}$ ashing; (7) after the cleaning with $\mathrm{HNO}_{3}$.

More detailed analysis is performed for the molecular exchange of OPE3 by OPV2 molecules in Au NPs bridged junctions (Fig. 2.12), which shows that for the average of more than 10 samples the tendency of the decreasing resistance remains the same [16]. 


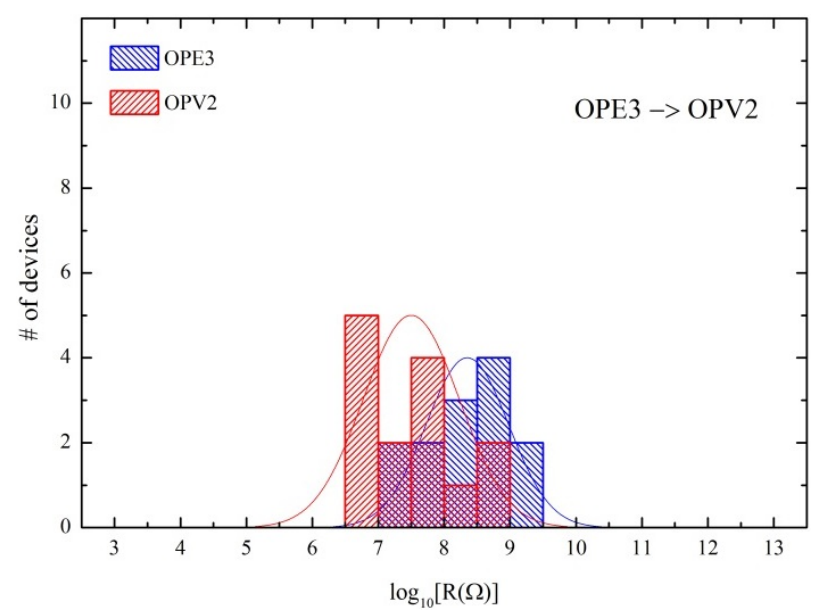

Figure 2.12. Statistical histograms of $\log _{10}[R(\Omega)]$ measured at $0.1 \mathrm{~V}$ for $40 \mathrm{~nm}$ Au junctions bridged by $60 \mathrm{~nm}$ Au NPs and functionalized by S,S' [1,4-Phenylenebis(2,1-ethynediyl-4,1-phenylene)]bis(thioacetate) (blue) and later exchanged by 4,4'-dimercaptostilbene (red).

\subsection{Single-Electron Transistor}

Low temperature measurements reveal another advantage of the DEP. NPs trapping between electrodes functionalized by molecular SAMs allows us to create a SET where SAMs play a role as tunnel barriers between electrodes and NPs. Charge transport measurements at a temperature of $22 \mathrm{mK}$ show a clear Coulomb blockade and Coulomb diamonds, demonstrating that the transport is from two or more NPs (see chapter 5).

$\mathrm{An} \mathrm{Au} \mathrm{AB}$ ring containing $40 \mathrm{~nm}$ gap between the ring and the interconnecting leads was functionalized by 1,6-hexanedithiol and $60 \mathrm{~nm} \mathrm{Au}$ NPs were trapped in the gap. Figure 2.13 represents the differential conductance $(\mathrm{d} I / \mathrm{d} V)$ as a function of source-drain voltage $\left(V_{\mathrm{SD}}\right)$ and back-gate voltage $\left(V_{G}\right)$ at a temperature of $22 \mathrm{mK}$ (as a back gate $\mathrm{p}++\mathrm{Si}$ is used underneath $35 \mathrm{~nm}$ of $\mathrm{SiO}_{2}$ ). The measurements were performed in a cryogenfree dilution refrigerator in a two point configuration using a low-noise technique (Section 1.8). Charging energy of the SET is about $E_{\mathrm{C}}=0.7 \mathrm{meV}$.

The advantage of the SET fabrication via DEP is that the charging energy of the device can be tuned by careful choice of the molecular SAM and the type and size of the NPs. Using molecular exchange, simply by dipping the device in a fresh solution with new molecules, the charging energy can be significantly changed without building up a new device (Chapter 4). 
Furthermore, by choosing smaller NPs or semiconductor NPs, it is possible to reveal more fundamental physical effects [19].
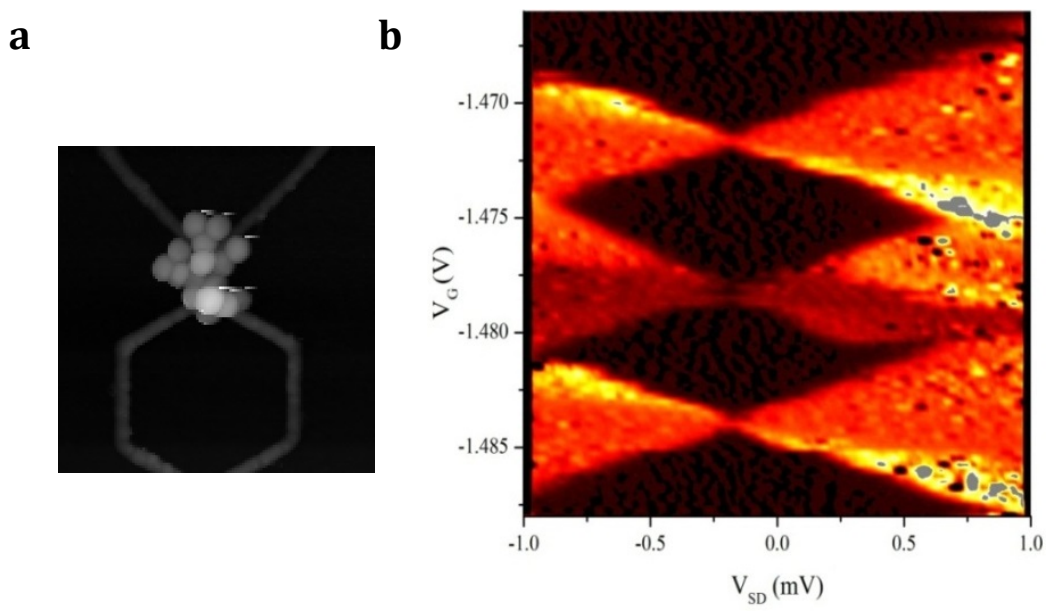

Figure 2.13. a, AFM image of the Au ring containing a gap on the side bridged by $60 \mathrm{~nm}$ Au NPs. b, Differential conductance (dl/dV) of the device in a as a function of the source-drain voltage $\left(V_{S D}\right)$ and back gate voltage $\left(V_{G}\right)$ at a temperature of $22 \mathrm{mK}(\mathrm{B}=8 \mathrm{~T})$.

\subsection{Evolution of the Designless Nanoparticle Network into Boolean Logic}

By increasing trapping time and NPs concentration in a solution during DEP procedure it is possible to create an isolated designless NPs network. Figure $2.14 \mathrm{~b}$ shows an example of such disordered network of $20 \mathrm{~nm} \mathrm{Au}$ NPs trapped in a circular region (200 $\mathrm{nm}$ in diameter) between twelve metal electrodes (Fig. 2.14a). For such electrode configuration NPs trapping is performed sequentially with pairs of diametrically opposite electrodes, by contacting the pads with the probe-station needles.

Based on the idea of the fabrication of disordered NPs network via NPs trapping Bose, Lawrence et al. [20] experimentally shown that below the temperature of $5 \mathrm{~K}$, when each particle isolated by 1-octanethiol acts as a SET, a network of randomly assembled Au NPs can be configured in situ into any of the commonly used 2-input Boolean logic gates by a small set of control voltages. 
$\mathbf{a}$

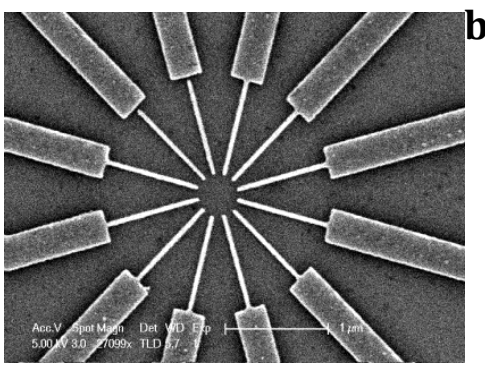

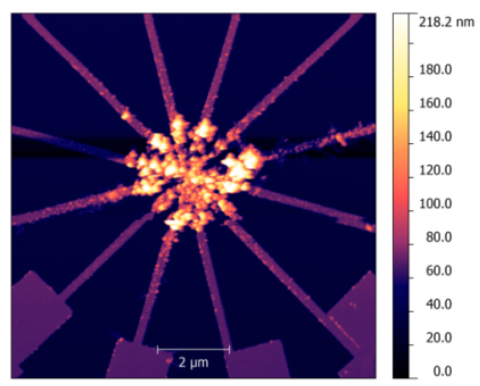

Figure 2.14. NP network. a, SEM image of 12 Au electrodes with an inner spacing of $200 \mathrm{~nm}$. b, AFM image of $20 \mathrm{~nm}$ Au NPs trapped between electrodes depicted in $\mathbf{a}$.

\section{Conclusions}

DEP is a high yield, simple and reproducible processing technique for combining top-down and bottom-up fabrication approaches. It is a quick and easy method which allows electrical characterisation of a large number of molecules and exchanging molecules in the junction. Electrical measurements shown that the electron transport weakly depends on the external parameters such temperature and magnetic field. On the other hand, molecule's characteristics (such as length and chemical composition) play an important role in the transport. An increase of the molecule length causes an exponential decrease in the molecule's conductance. By varying the chemical composition it is possible to change the resistance through the molecular junction from GigaOhms for long non-conjugated molecules to kiloOhms for short or conjugated ones. Performed experiments show that molecules in the molecular junction can be exchanged without fabrication of a new device. The Coulomb blockade regime can be achieved at cryogenic temperatures in a metal-molecule-NP-molecule-metal junction created via DEP. The obtained results show that the used DEP technique is an effective and easy method for studying different molecular layers and can be used for investigating charge transport through the molecular junctions. 


\section{REFERENCES}

[1] M. A. Reed, C. Zhou, C. J. Muller, T. P. Burgin, and J. M. Tour, "Conductance of a Molecular Junction," Science, vol. 278, pp. 252-254, October 10, 19971997.

[2] D. C. Ralph, C. T. Black, and M. Tinkham, "Spectroscopic Measurements of Discrete Electronic States in Single Metal Particles," Physical Review Letters, vol. 74, pp. 3241-3244, 04/17/ 1995.

[3] D. C. Ralph, C. T. Black, and M. Tinkham, "Gate-Voltage Studies of Discrete Electronic States in Aluminum Nanoparticles," Physical Review Letters, vol. 78, pp. 4087-4090, 05/26/ 1997.

[4] J. Park, A. N. Pasupathy, J. I. Goldsmith, C. Chang, Y. Yaish, J. R. Petta, et al., "Coulomb blockade and the Kondo effect in single-atom transistors," Nature, vol. 417, pp. 722-725, 06/13/print 2002.

[5] S. Kubatkin, A. Danilov, M. Hjort, J. Cornil, J. L. Bredas, N. StuhrHansen, et al., "Single-electron transistor of a single organic molecule with access to several redox states," Nature, vol. 425, pp. 698-701, Oct 162003.

[6] B. Xu and N. J. Tao, "Measurement of Single-Molecule Resistance by Repeated Formation of Molecular Junctions," Science, vol. 301, pp. 12211223, August 29, 20032003.

[7] I. Amlani, A. M. Rawlett, L. A. Nagahara, and R. K. Tsui, "An approach to transport measurements of electronic molecules," Applied Physics Letters, vol. 80, pp. 2761-2763, 2002.

[8] A. Kuzyk, "Dielectrophoresis at the nanoscale," Electrophoresis, vol. 32, pp. 2307-2313, 2011.

[9] H. A. Pohl, Dielectrophoresis : the behavior of neutral matter in nonuniform electric fields. Cambridge; New York: Cambridge University Press, 1978.

[10] D. Cheon, S. Kumar, and G.-H. Kim, "Assembly of gold nanoparticles of different diameters between nanogap electrodes," Applied Physics Letters, vol. 96, pp. -, 2010.

[11] H. B. Akkerman, P. W. M. Blom, D. M. de Leeuw, and B. de Boer, "Towards molecular electronics with large-area molecular junctions," Nature, vol. 441, pp. 69-72, 05/04/print 2006.

[12] C. Chu, J. S. Na, and G. N. Parsons, "Conductivity in alkylamine/gold and alkanethiol/gold molecular junctions measured in molecule/nanoparticle/molecule bridges and conducting probe structures," Journal of American Chemical Society, vol. 129, pp. 2287-96, Feb 28 2007.

[13] G. Wang, T.-W. Kim, and T. Lee, "Electrical transport characteristics through molecular layers," Journal of Materials Chemistry, vol. 21, pp. 18117-18136, 2011. 
[14] M. M. Thuo, W. F. Reus, C. A. Nijhuis, J. R. Barber, C. Kim, M. D. Schulz, et al., "Odd-Even Effects in Charge Transport across SelfAssembled Monolayers," Journal of the American Chemical Society, vol. 133, pp. 2962-2975, 2011/03/09 2011.

[15] H. B. Akkerman and B. d. Boer, "Electrical conduction through single molecules and self-assembled monolayers," Journal of Physics: Condensed Matter, vol. 20, p. 013001, 2008.

[16] T. T. Liang, Y. Naitoh, M. Horikawa, T. Ishida, and W. Mizutani, "Fabrication of steady junctions consisting of alpha,omega-bis(thioacetate) oligo(p-phenylene vinylene)s in nanogap electrodes," Journal of the American Chemical Society, vol. 128, pp. 13720-6, Oct 252006.

[17] D. P. Long, C. H. Patterson, M. H. Moore, D. S. Seferos, G. C. Bazan, and J. G. Kushmerick, "Magnetic directed assembly of molecular junctions," Applied Physics Letters, vol. 86, pp. -, 2005.

[18] L. Bernard, Y. Kamdzhilov, M. Calame, S. J. van der Molen, J. Liao, and C. Schönenberger, "Spectroscopy of Molecular Junction Networks Obtained by Place Exchange in 2D Nanoparticle Arrays," The Journal of Physical Chemistry C, vol. 111, pp. 18445-18450, 2007/12/01 2007.

[19] F. Kuemmeth, K. I. Bolotin, S.-F. Shi, and D. C. Ralph, "Measurement of Discrete Energy-Level Spectra in Individual Chemically Synthesized Gold Nanoparticles," Nano Letters, vol. 8, pp. 4506-4512, 2008/12/10 2008.

[20] S. K. Bose, C. P. Lawrence, Z. Liu, K. S. Makarenko, R. M. J. van Damme, H. J. Broersma, et al., "Evolution of a Designless Nanomaterials System into Boolean Logic," in preparation. 
Chapter 2 


\section{Chapter 3 Wedging Transfer TeChNique}

This chapter is dedicated to the experimental investigation of the wedging transfer technique and implementation of the method for soft landing of an electrical contact on top of the bottom electrode functionalized by a selfassembled monolayer (SAM) in order to create nanoscopic molecular junctions for the investigation of the charge transport through organic monolayers. Electrical characterisation was employed to test charge transport dependence on the temperature and molecule's length. We also compare properties of molecular junctions made via wedging transfer technique and dielectrophoresis (DEP) manipulation method. 
The biggest challenge for studying charge transport through molecular layers is the creation of molecular junctions. In chapter 2 we have discussed the investigation of molecular junctions fabricated via DEP manipulation method. Particularly, the nanoparticles (NPs) trapping technique was tested. In this chapter we explore wedging transfer as an alternative method for the fabrication of nanosccopic molecular junctions. Via this technique a top electrical contact can be soft-landed on top of the bottom electrode functionalized by SAM avoiding direct metal evaporation which can lead to metal filaments piercing through the monolayer causing shorts $[1,2]$.

\subsection{Wedging Transfer as an Alternative Method for the Creation of Molecular Junctions}

Wedging transfer is a relatively new technique for transferring nano- and microstructures of various shapes and compositions onto different types of surfaces [3-5]. In the basis of the method lies the hydrophilic/hydrophobic effect: water "likes" to wet hydrophilic surfaces and avoid hydrophobic ones. When a hydrophilic substrate containing a hydrophilic object is immersed in water, water lifts-off the object from the substrate.

In 2013, Krabbenborg, Wilbers et al. applied wedging transfer for the first time for fabrication and electrical characterisation of the large-area (100$400 \mu^{2}$ ) metal-molecular monolayer-metal junctions [4]. In this thesis the method is adopted for the creation of molecular junctions at nanoscale. The schematic of the wedging transfer is depicted in fig. 3.1. The process consist of the following steps:

- a hydrophilic substrate containing a hydrophobic object is treated by ozone to increase hydrophilicity of the surface;

- a hydrophobic polymer is dip coated on the top of the sample and let dry;

- substrate is immersed in water where polymer is lifted-off pilling off the object;

- floating on water surface polymer containing the object is aligned to a preliminary cleaned nanogap and functionalized by the SAM while water is slowly pumped out;

- bringing together the object covered by a polymer and the SAM-covered nanogap;

- dissolving the polymer. 


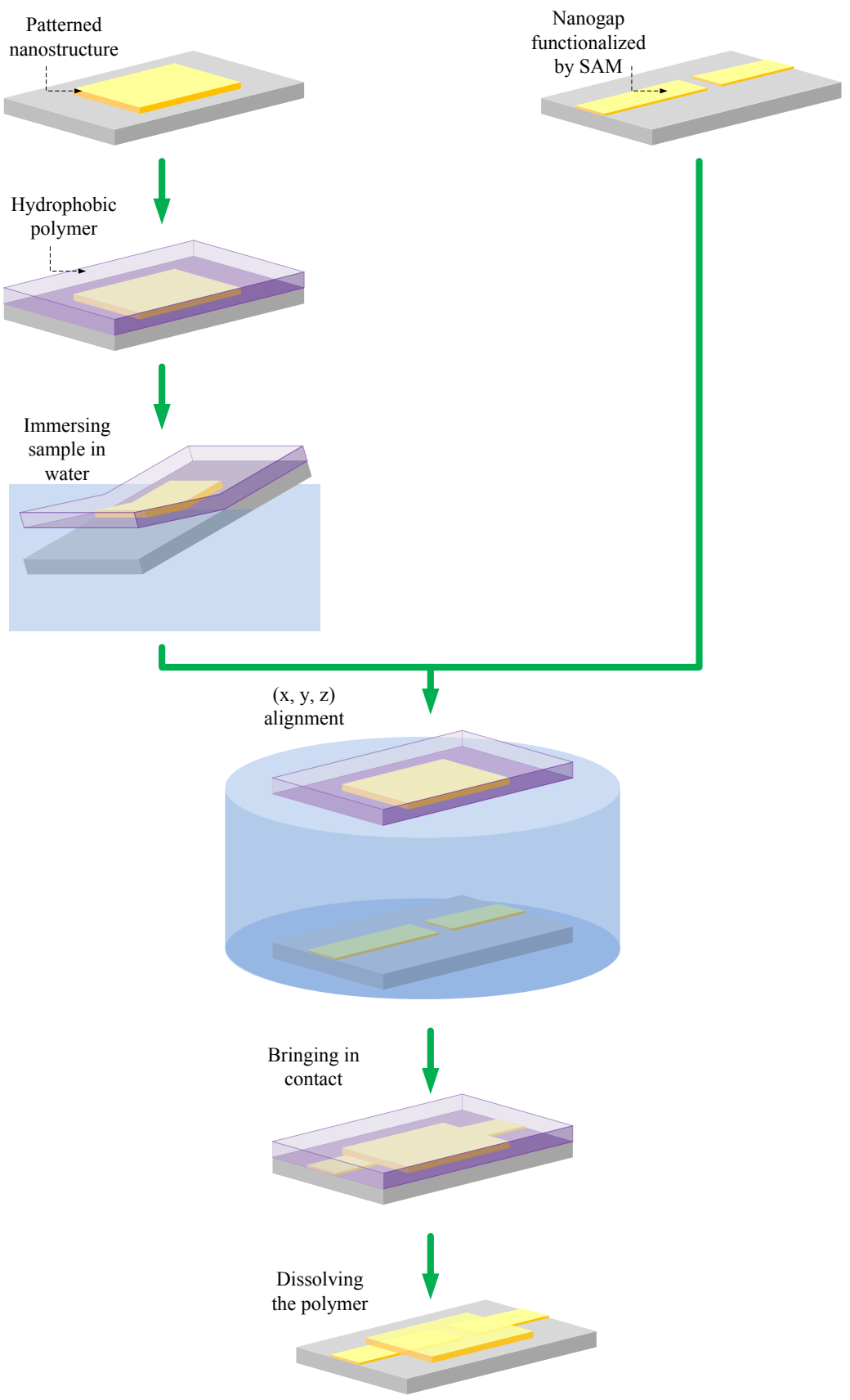

Figure 3.1. Schematic of the wedging transfer process. 


\subsection{Device Fabrication}

To increase the yield of well-aligned devices the design of the nanogaps and dots "to-be-transferred" has to be chosen carefully due the difficulty of the alignment procedure (see below) of the floating polymer to the substrate containing nanogaps. If the distance between dots is $s$, then the first two nanogaps should be shifted in $y$-direction by $s / 2$ relatively to each other, while the second two nanogaps should be shifted by $s / 2$ in $x$-direction relatively to the first two gaps (Fig. 3.2).

a

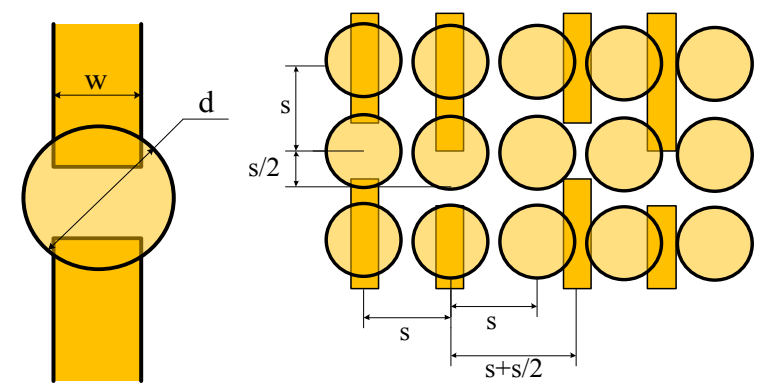

b

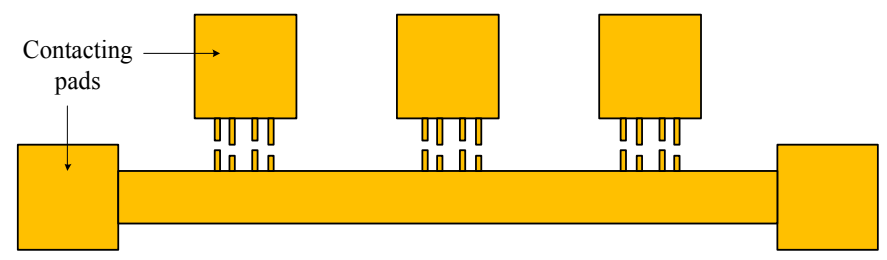

Figure 3.2. Schematic of the possible design of the junctions and transferred dots (depicted as circles).

A group of four gaps is repeated 60 times in a matrix of $5 \times 12$ groups on one $11 \times 11 \mathrm{~mm}^{2} \mathrm{Si} / \mathrm{SiO}_{2}$ substrate. Nanodots ( $<s$ in diameter, spaced by the distance $s$ ) are patterned in sixty $150 \times 150 \mu \mathrm{m}^{2}$ square areas repeating the design of nanogaps. Ideally, during the alignment procedure one out of four gaps should be "hit" by an island. The amount of built dots is limited by electron beam lithography writing time.

Atomic force microscopy images showing experimental results of the wedging transfer fabrication process are depicted in fig. 3.3. Here, $200 \times 200$ $\mathrm{nm}^{2} \mathrm{Au}$ dots (Fig. 3.3b) are transferred on top of $40 \mathrm{~nm}$ Au gaps functionalized by a SAM (Fig. 3.3a), resulting in the creation of metal-molecular monolayermetal nanojunctions (Fig. 3.3c). As can been seen in the fig. 3.3c, one $40 \mathrm{~nm} \mathrm{Au}$ gap is covered by $200 \times 200 \mathrm{~nm}^{2} \mathrm{Au}$ island. 
$\mathbf{a}$

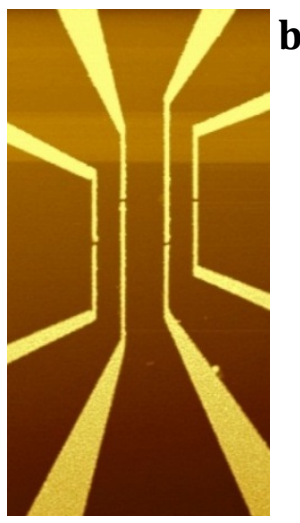

b

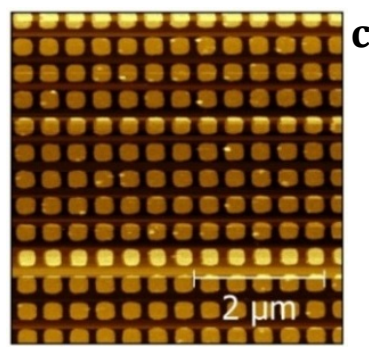

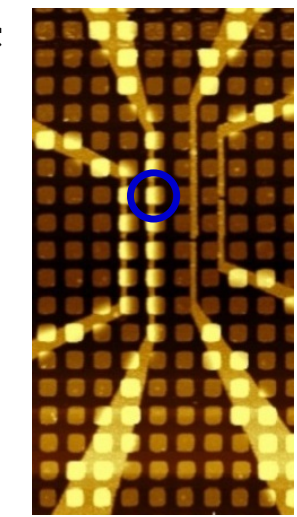

Figure 3.3. AFM images showing the wedging transfer fabrication process. a, $40 \mathrm{~nm}$ Au nanogaps; b, $200 \mathrm{~nm}$ square Au dots "to be transferred" on top of the nanogaps in $\mathbf{a} ; \mathbf{c}$, Au dots in $\mathbf{b}$ transferred on top of Au nanogaps in a (high-lighted by blue circle).

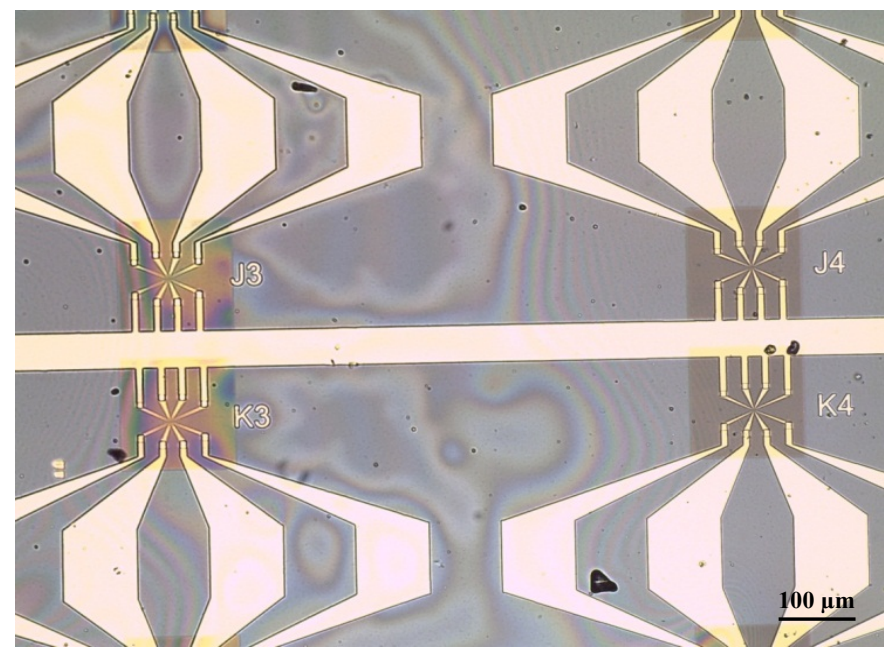

Figure 3.4. Optical image of the $200 \mathrm{~nm}$ square Au dots attached to the cellulose floating in water above a $\mathrm{Si} / \mathrm{SiO}_{2}$ substrate, containing $40 \mathrm{~nm}$ Au nanogaps covered by self-assembled monolayer. 
The optical image in Fig. 3.4 shows the alignment procedure of the floating-on-water-polymer with $150 \times 150 \mu \mathrm{m}^{2}$ square areas containing $\mathrm{Au}$ $200 \times 200 \mathrm{~nm}^{2}$ square islands on top of groups of four $40 \mathrm{~nm} \mathrm{Au} \mathrm{gaps}$ connected to the $100 \times 100 \mu \mathrm{m}^{2}$ contacting pads (used to connect probes for the electrical characterisation). Due to the micrometer-sized features a microscope lens with a short focal distance has to be used. That leads to difficulties for the use of the manipulation probe needles and water-pumping syringes. As a result the alignment has to be performed by hand what can influence the quality and the yield of the working devices.

\subsection{Molecular Length Dependence}

Immidiately after the device fabrication (to prevent degradation of the molecular SAM) electrical characterisation of nanojunctions is performed in vacuum $\left(<10^{-4} \mathrm{mbar}\right)$ first at room and then low $(5 \mathrm{~K})$ temperatures. Currentvoltage $(I-V)$ measurements are carried out in a probe station in a two-probe configuration using Keithley 2400 SourceMeter and controlled via custommade LabView-based program.

In this thesis for the examination of the molecular length dependence, molecular junctions containing alkanethiol SAMs with six, eight and ten

Table 3.1. Working device yield for measurements of molecular junctions with alkanethiol SAMs of different length $\left(N_{\mathrm{C}}\right)$

\begin{tabular}{c|c|c}
\hline$N_{\mathrm{C}}$ & $\begin{array}{c}\text { Total number } \\
\text { of junctions }\end{array}$ & Yield, \% \\
\hline 6 & 60 & 45 \\
8 & 120 & 23 \\
10 & 120 & 5 \\
\hline
\end{tabular}
carbons are fabricated and electrically characterised. Only devices showing non-linear Simmons behaviour [6-8] are considered as "working" (Table 3.1) and are represented in statistical histograms of $\log _{10}[R(\Omega)] \quad(R$ is the resistance of the device) (Figs. 3.5-3.6). Based on the measured resistance, the devices are divided into "non-working" (when a short is measured or the signal is in the noise level) and "working". The data of the "non-working" devices is excluded from this thesis. The yield of the "working" devices is varying for different molecules. Due to the extremely small $(\sim 5 \%)$ yield of the "working" devices for the 1,10-decanedithiol, statistical analysis of the experimental data cannot be performed and is excluded from this thesis.

Figures 3.5-3.6 represent statistical histograms of $\log _{10}[R(\Omega)]$ measured at $0.1 \mathrm{~V}$ for the junctions created via the wedging transfer technique and functionalized by 1,6-hexanedithiol and 1,8-octanedithiol, and based on the data from "working" devices. Unfortunately, due to the lack of data for 
1,10-decanedithiol, the length dependence cannot be plotted. Yet, comparison of the data in Figs. 3.5-3.6 to the semilog plot in Fig. 2.9 shows that molecular SAMs junctions created via wedging transfer technique are more conducting than junctions fabricated by means of NP bridge, as we will further discuss in the next section.

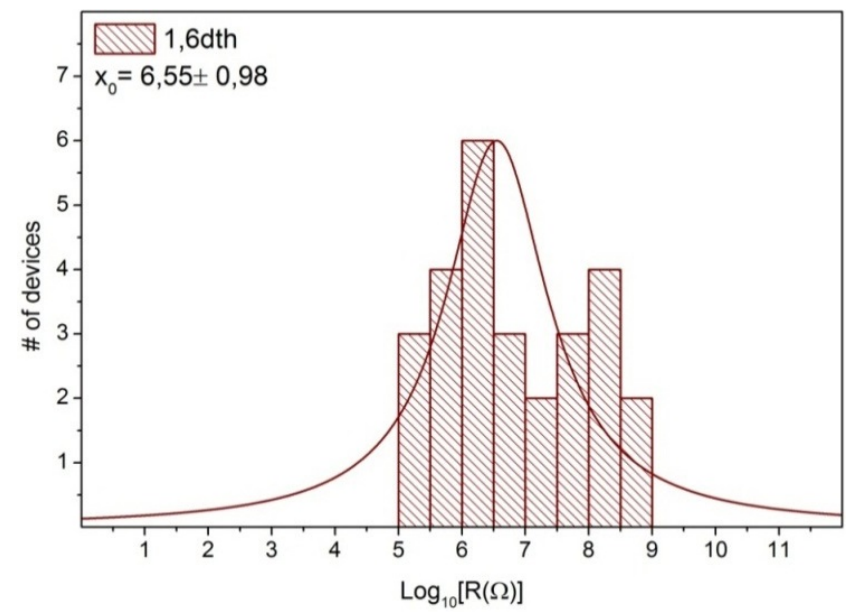

Figure 3.5. Statistical histograms of $\log _{10}[R(\Omega)]$ measured at $0.1 \mathrm{~V}$ for the $40 \mathrm{~nm}$ Au junctions functionalized by 1,6-hexanedithiol and bridged by $200 \times 200 \mu \mathrm{m}^{2}$ square Au dots.

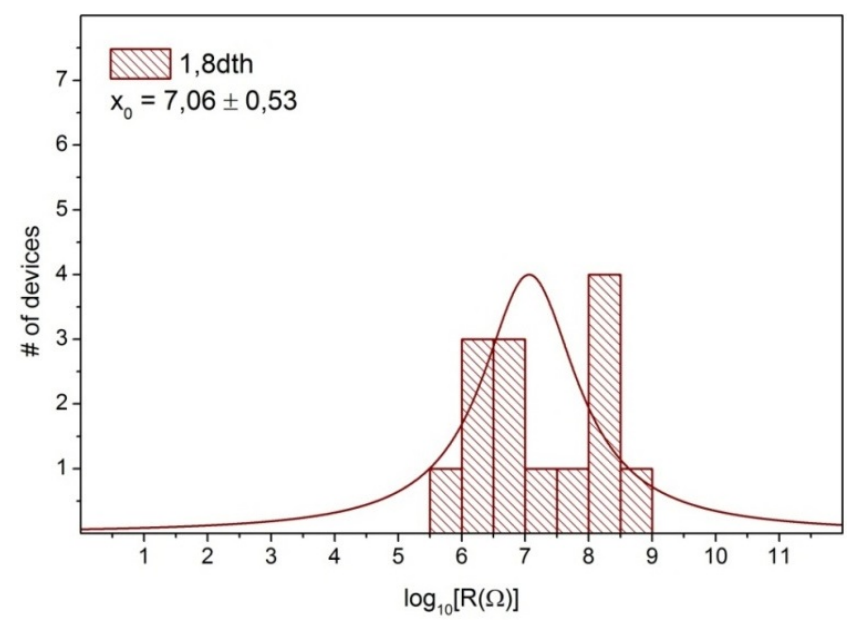

Figure 3.6. Statistical histograms of $\log _{10}[R(\Omega)]$ measured at $0.1 \mathrm{~V}$ for the $40 \mathrm{~nm} \mathrm{Au}$ junctions functionalized by 1,10 -octanedithiol and bridged by $200 \times 200 \mu \mathrm{m}^{2}$ square Au dots. 


\subsection{Comparison of the Molecular Junctions Made via Nanoparticle Bridge to the Molecular Junctions Created via Wedging Transfer Technique}

Relying on the data obtained from the electrical measurements on the molecular junctions created via NP bridging (Chapter 2) and wedging transfer a comparison between two techniques can be performed. Schematics of the molecular junctions created by means of a NP bridge and wedging transfer are depicted in Fig. 3.7.

As it was discussed in chapter 1 non-resonant tunnelling charge transport through molecular SAMs is proportional to the effective contact resistance $R_{0}$ (Eq. 1.10) which depends on the anchoring group and the contact electrodes. In the case of alkanedithiols the sulfhydryl (-SH) group is used as an anchoring group to attach the alkane chain to gold. Nevertheless, the interface between SAMs and electrodes plays a crucial role influencing the coupling of the anchoring group to the metal.
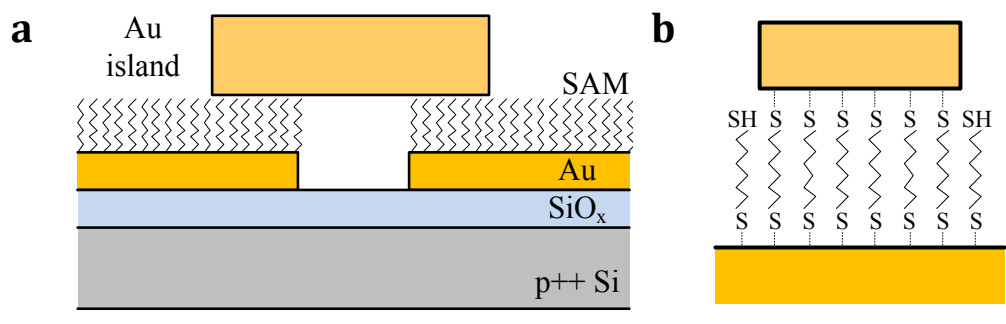

c

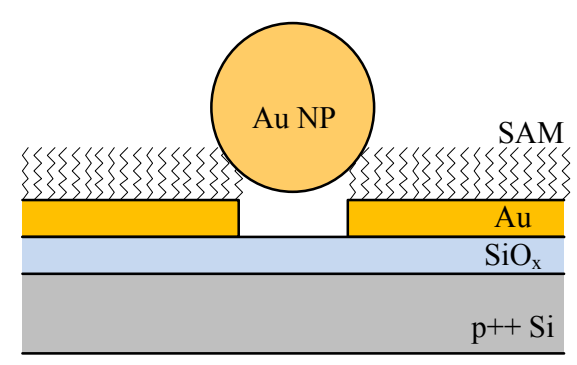

d
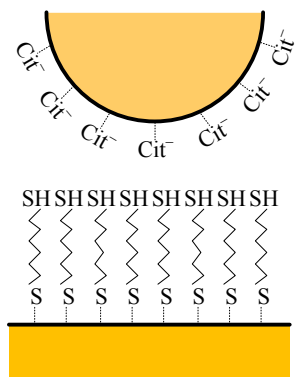

Figure 3.7. Schematics of the molecular junctions created via wedging transfer (a-b) and via nanoparticle bridge (c-d). Cit represents citrate molecules.

Prior to the wedging transfer the top electrode is electron-beam evaporated on top of thermally grown $\mathrm{SiO}_{2}$, what leads to the formation of a very flat bottom surface of the top electrode [4] which is later landed on top of 
the molecular SAM during wedging transfer procedure (Fig. 3.7a,b). However, most of commercially Au NPs are covered by citrate layer which is stabilizing the particles in solution. Thereby, when NP is placed on top of the molecular SAM there is a citrate layer between the SAM and the NP which can lead to bad coupling of the molecules to the metal (Figs. 3.7c,d). This might explain lower resistance measured in devices made via wedging transfer compared to the NPs bridges (Figs. 2.8, 3.5-3.6). Another reason could be that the cross-section area of the molecular junction created via wedging transfer is bigger than the area of NP bridged junctions.

Another characteristic differing NP bridging from wedging transfer technique is a possibility to build a single-electron transistor. The charging energy is inversely proportional to the capacitance of an object which scales with its radius [9]. Commercially available NPs are not bigger than $120 \mathrm{~nm}$ in diameter while the size of the nanoisland, fabricated via the wedging transfer technique, is unlimited and determined by experimentalist's design.

\section{Conclusions}

Wedging transfer is a promising technique for the fabrication and electrical characterisation of nanoscopic molecular junctions. Electrical characterisation demonstrated that the junctions created via wedging transfer are more conductive than junctions fabricated via NPs bridging. The yield of the working devices was shown to be lower than $50 \%$ which could be improved, for example, by the immersing of the bottom electrode into $\mathrm{SiO}_{2}$ to flatten the edges or by using metal with a smoother surface. Due to the expected low charging energies of the transferred metallic islands, wedging transfer has an advantage when low temperature measurements on molecular layer have to be done.

\section{REFERENCES}

[1] K. Tae-Wook, W. Gunuk, L. Hyoyoung, and L. Takhee, "Statistical analysis of electronic properties of alkanethiols in metal-molecule-metal junctions," Nanotechnology, vol. 18, p. 315204, 2007.

[2] G. S. Bang, H. Chang, J. R. Koo, T. Lee, R. C. Advincula, and H. Lee, "High-fidelity formation of a molecular-junction device using a thicknesscontrolled bilayer architecture," Small, vol. 4, pp. 1399-405, Sep 2008. 
[3] G. g. F. Schneider, V. E. Calado, H. Zandbergen, L. M. K. Vandersypen, and C. Dekker, "Wedging Transfer of Nanostructures," Nano Letters, vol. 10, pp. 1912-1916, 2010/05/12 2010.

[4] S. O. Krabbenborg, J. G. E. Wilbers, J. Huskens, and W. G. van der Wiel, "Symmetric Large-Area Metal-Molecular Monolayer-Metal Junctions by Wedging Transfer," Advanced Functional Materials, vol. 23, pp. 770-776, 2013.

[5] V. E. Calado, G. F. Schneider, A. M. M. G. Theulings, C. Dekker, and L. M. K. Vandersypen, "Formation and control of wrinkles in graphene by the wedging transfer method," Applied Physics Letters, vol. 101, pp. -, 2012.

[6] H. B. Akkerman, P. W. M. Blom, D. M. de Leeuw, and B. de Boer, "Towards molecular electronics with large-area molecular junctions," Nature, vol. 441, pp. 69-72, 05/04/print 2006.

[7] J. G. Simmons, "Generalized Formula for the Electric Tunnel Effect between Similar Electrodes Separated by a Thin Insulating Film," Journal of Applied Physics, vol. 34, pp. 1793-1803, 1963.

[8] G. Wang, T.-W. Kim, and T. Lee, "Electrical transport characteristics through molecular layers," Journal of Materials Chemistry, vol. 21, pp. 18117-18136, 2011.

[9] C. Harmans and F. d. T. N. TU Delft, Mesoscopic Physics: An Introduction : TN385 [dictaat Behorende Bij College TN3853]: TU Delft, 2003. 


\section{Chapter 4 BotTOM-UP INORGANIC-ORGANIC SingLE-ELECTRON TRANSISTORS}

In this chapter, we report a new reproducible technique for the fabrication of single-electron transistors (SETs) based on Au nanoparticles (NPS). We combine top-down lithography and bottom-up self-assembly of Au NPs and Au nanorods (NRs), bridging the gap between the nano- and microscale. By means of selective chemical interactions, functionalised $A u$ NRs and $A u$ NPs in dispersion selfassemble into NR-NP-NR assemblies. The NR-NP-NR assemblies are deposited on a highly doped $\mathrm{Si} / \mathrm{SiO}_{2}$ substrate and electrically contacted with metal electrodes defined by electron beam lithography (EBL) obtaining SETs with the substrate acting as a back gate. After the device fabrication, we replace the linker molecules (homocysteine) between the NPs and NRs with 1,8-octanedithiol. The molecular barrier decouples single NP from the two NR-contacts, as demonstrated by the clear Coulomb blockade features observed at lowtemperature $(<15 \mathrm{~K})$ in the charge-transport measurements.

\footnotetext{
${ }^{*}$ K.S. Makarenko ${ }^{\dagger}$, Z. Liu $^{\dagger}$ et al, in preparation
} 


\subsection{Self-Assembly of a Single Nanoparticle to two Gold Nanorods}

Over the last few decades semiconductor electronics has evolved extremely successfully, based on the miniaturization of silicon transistors. This ongoing miniaturization is largely enabled by the developments in top-down fabrication methods. However, as we are approaching critical features of $\sim 10$ $\mathrm{nm}$, top-down fabrication becomes so challenging and costly that alternative fabrication schemes are most probably required. According to the International Technological Roadmap for Semiconductors (ITRS) electronic circuits will already reach this limit within a decade [1].

Connecting single nanostructures and molecules to electric circuitry has proven to be an extremely difficult task. Many (top-down) schemes have been developed [2-8]. These techniques, however, often suffer from serious intrinsic problems, such as poor reproducibility, lack of control of number and orientation of nanostructures and damage due to fabrication. The synthesis of inorganic materials with dimensions on the nanometer scale has greatly improved in recent years $[9,10]$. For example, hierarchical integration of nanoscale building blocks (nanoparticles, nanorods, nanotubes etc.) into functional assemblies, and further into functional devices is suggested to be achieved through a "bottom-up approach" [11-13]. Examples include dimer gold nanoparticles linked by organic dithiol molecules [11], the preparation of ordered metal-molecule-metal networks by photosensitive molecules [12], and photolithography linkage of $\mathrm{ZnO}$ nanowires [13]. Au NRs have gained much attention due to their wide range of applications as "interconnectors" in nanoscale devices [14]. End-to-end self-assembly of Au NRs has previously been reported through electrostatic interactions $[15,16], \mathrm{H}$-bonding/covalent bonding [17], and avidin-biotin interactions [18]. Similarly, one-dimensional self-assembly of Au NPs has also been reported driven by diverse chemical interactions [19-21].

Those initial one-component systems (component referring to the type of nanostructure involved) have already increased our understanding of particle-particle interactions on the nanometer scale, and also formed an inspiration for the development of our new way to prepare multi-component assemblies for the realization of nanoelectronic devices.

We proposed an unconventional way of realizing functional nanoelectronic devices (in particular SETs) using a chemical (bottom-up fabrication) approach based on self-assembly and chemical recognition (Fig. 4.1). We combine self-assembly and molecular recognition at nanoscale on one side with conventional top-down technology at larger scale on the other side which forms an innovative path to bridge the micro-nanogap. 
Compared to previous methods, our strategy has several advantages. First, a single $\mathrm{Au}$ NP is linked to two NRs of $\sim 250 \mathrm{~nm}$ length, making the total size of the assembly large enough to connect it relatively easily to electrodes using standard EBL. Second, the chemical linkage between the Au NP and the $\mathrm{Au}$ NRs is well-defined and tunable by chemical engineering. Third, there is great flexibility in choosing the nano objects to be electrically connected: a metallic nanoparticle, a semiconductor quantum dot or a single molecule. Last but not least, bottom-up assembly has in principle the potential to produce vast amounts of the desired assembly in parallel. Moreover, our approach is interesting from a chemistry point of view, in particular the control/guidance and limitation of self-assembly and complexity.

To the best of our knowledge, our specific approach - chemically connecting $\mathrm{Au}$ NPs to Au NRs - have never been used for the purpose of bottom-up fabricated nanoelectronic devices.

a
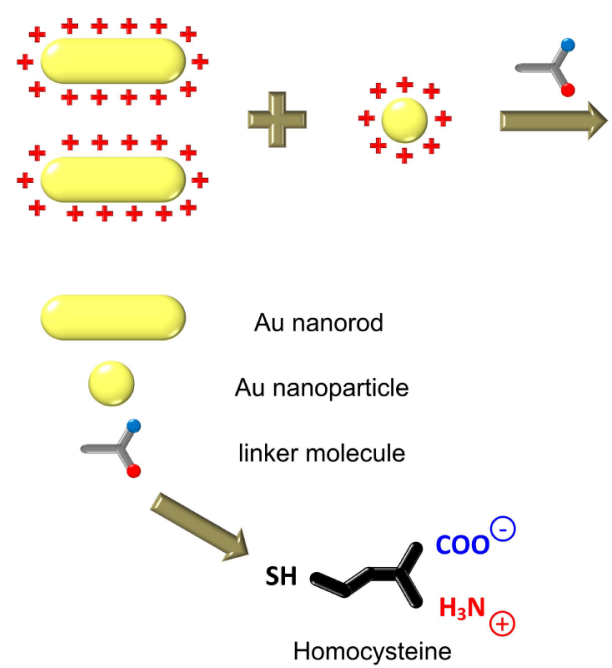

b
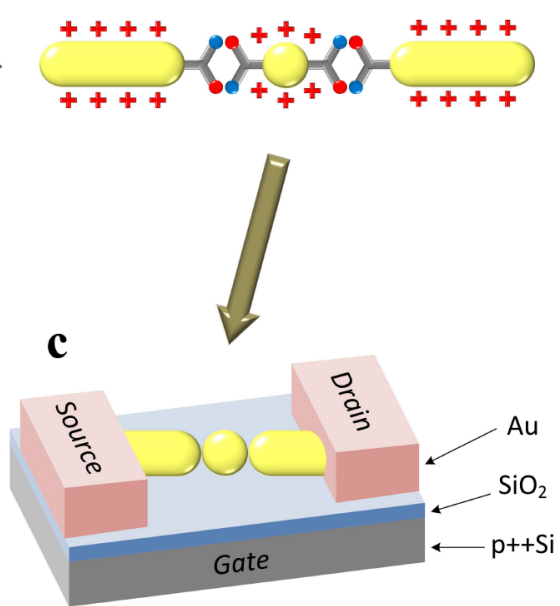

Figure 4.1. Schematic representation of the bottom-up self-assembly combined with top-down lithography to realize a SET based on a single Au NP. a-b, Formation of the NR-NP-NR assembly by means of selective chemical interactions and deposition of the assembly on a substrate; $\mathbf{c}$, contacting of the assembly with metal electrodes defined by EBL. 


\subsection{Sample Fabrication}

\subsubsection{Bottom-Up Approach*}

Studies of the Au NR crystal structure show that the end facets of Au NRs are dominated by (111) planes, and the side facets by (110) and (100) planes [2224]. Thiol groups preferentially bind to the $(111)$ planes $[17,18]$ at low concentration. Based on this selective modification of the Au NRs ends, the specific chemical interactions are expected with preferably the ends of the $\mathrm{Au}$ NRs. We propose that Au NRs might link with a single Au NP due to the selective chemical interactions. We tested a few methods (e.g., click-chemistry, "host"-"guest" interactions) to form the assemblies. Nevertheless, the highest yield was achieved with a homocysteine, a thiol-containing amino acid, as a linker molecule between Au NRs and Au NP. At pH 6.5, around the isoelectric point of homocysteine, it becomes zwitterionic. The zwitteronic groups at the $\mathrm{Au}$ NRs ends and the NP cause attraction through a two-point electrostatic attraction in a cooperative fashion (Fig. 4.1). The NP needs to react with thiols, so Au NPs were chosen.

Cetyltrimethylammonium bromide (CTAB) stabilized $\mathrm{Au}$ NPs were prepared by adding $200 \mu \mathrm{L}$ citrate stabilized Au NPs into $800 \mu \mathrm{L}$ CTAB aqueous solution $(25 \mathrm{mM})$ under ultra-sonication. For the assembly of the $\mathrm{Au}$ NRs with NPs, $5 \mu \mathrm{L}$ CTAB stabilized Au NPs were mixed with $200 \mu \mathrm{L}$ Au NRs under shaking, then $2 \mu \mathrm{L}$ fresh homocysteine aqueous solution $(1 \mathrm{mM})$ were added into the colloidal solution. The dispersion was shaken and then kept at room temperature for 2 hours.

To prepare the sample for top-down lithography, the mixed dispersion was transferred onto a $\mathrm{Si} / \mathrm{SiO}_{2}$ substrate with markers (Figs. 1.9, 4.2a) cleaned for $10 \mathrm{~min}$ with oxygen plasma. The sample was left for 20 minutes for NR-NPNR to precipitate. Afterwards, the dispersion containing non-precipitated assemblies was blown away by high pressure nitrogen gas. Subsequently, the substrate was immersed in $5 \mathrm{mM}$ 1,8-octanedithiol ethanol solution for 24 hours to ensure that all of the homocysteine molecules were exchanged by 1.8-octaneditiol (we believe that homocysteine might not survive the fabrication procedure). Prior to EBL, the substrate was taken out from the dithiol solution and washed by a large amount of ethanol and dried by nitrogen gas (Fig. 4.2a).

\footnotetext{
* The bottom-up approach was realized by Zhihua Liu
} 
a

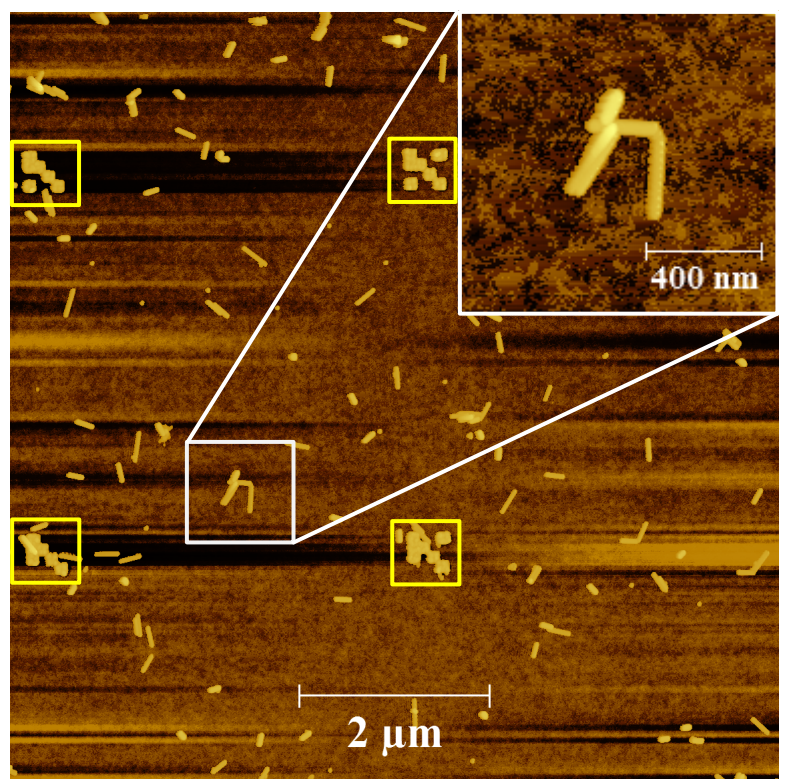

b

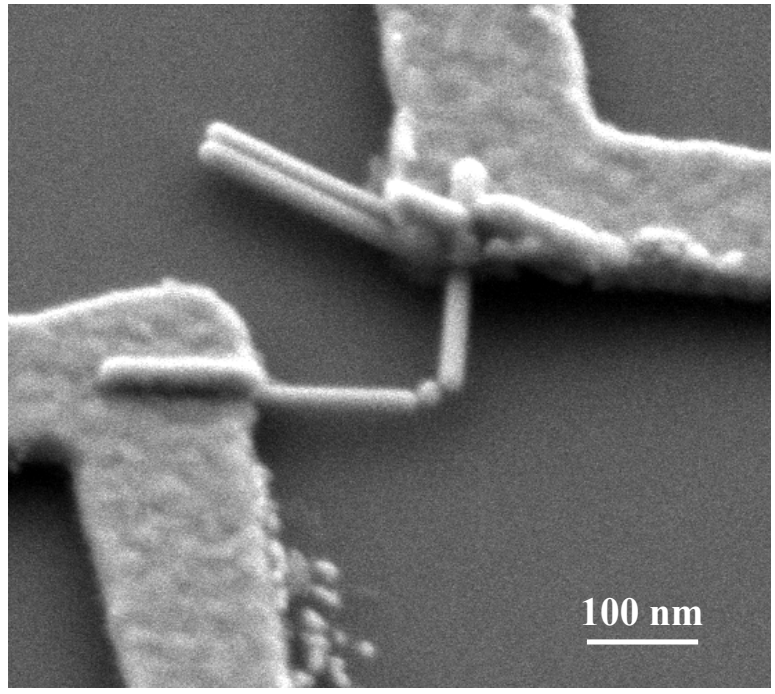

Figure 4.2. Bottom-up self-assembled SET. a, AFM image of the NR-NP$\mathrm{NR}$ assemblies deposited on a $\mathrm{Si} / \mathrm{SiO}_{2}$ wafer containing pre-patterned bitmap markers (inside yellow squares). Inset shows an assembly chosen for contacting. b, SEM image of the device (inset in a) contacted with EBL. 


\subsubsection{Top-Down Approach}

After the deposition of the NR-NP-NR assemblies, samples were imaged by AFM in order to find candidates for the EBL contacting. (In section 1.6.2 it was discussed why scanning electron microscopy (SEM) has to be avoided). Figure 4.2a shows a typical AFM image, where NR-NP-NR assemblies and bitmap markers (high-lighted by yellow squares) can be seen. The AFM images of selected candidates were used for the EBL design of the contacting electrodes. After the design preparation, samples were covered by $220 \mathrm{~nm}$ thick resist PMMA 950 A4. Our experiments have shown that prebaking of the resist resulted in a formation of short circuits between NRs and NPs. To avoid this, samples were left over night for the degassing of the resist solvent on a wet bench covered by a glass beaker and with a nitrogen flow access. Afterwards, an EBL was performed to define the contacts (Chapter 1).

In order to reach an EBL alignment accuracy better than $20 \mathrm{~nm}$, we used alignment markers (Section 1.6.1). Just prior to the metal evaporation the samples were cleaned with an oxygen plasma (in order to remove resist residuals) and rinsed in ethanol (for the removal of gold oxide). Immediately after metal evaporation ( $40 \mathrm{~nm}$ of Au on top of $3 \mathrm{~nm}$ Ti) samples were dipped into $5 \mathrm{mM} \mathrm{1,8-octanedithiol} \mathrm{acetone} \mathrm{solution} \mathrm{for} \mathrm{a} \mathrm{lift-off} \mathrm{procedure.} \mathrm{Following}$ the lift-off, samples were bonded and electrically tested. Figure $4.2 \mathrm{~b}$ shows an electrically contacted SET (one out of more than 15 devices) illustrated in the inset of Fig. 4.2a.

\subsection{Electron Transport through Bottom-up Self-Assembled Single- Electron Transistors}

\subsubsection{Self-Assembled Single Electron Transistors of Different Sizes}

Low-noise low-temperature measurements reveal a classical Coulomb blockade regime. Figure 4.3 shows a differential conductance $(\mathrm{d} I / \mathrm{d} V)$ as a function of the source-drain voltage $\left(V_{\text {bias }}\right)$ and back gate voltage $\left(V_{\text {gate }}\right)$ at temperature $<15 \mathrm{mK}$, showing the electron transport through SETs of different sizes. Around zero bias the conductance is suppressed and can be controlled by applying $V_{\text {bias }}$ and $V_{\text {gate. }}$. The charging energy of a Coulomb island is inversely proportional to the size of the island [25]. Our experiments have shown this trend. From Figs. 4.3a,b it can be seen that the smaller particle gives much bigger charging energy $(\sim 25.8 \mathrm{meV}$ for the $20 \mathrm{~nm} \mathrm{Au} \mathrm{NP}$ and 8.14 $\mathrm{meV}$ for the $\sim 100 \mathrm{~nm}$ Au flake). Calculated coupling parameters are shown in Table 4.1. 

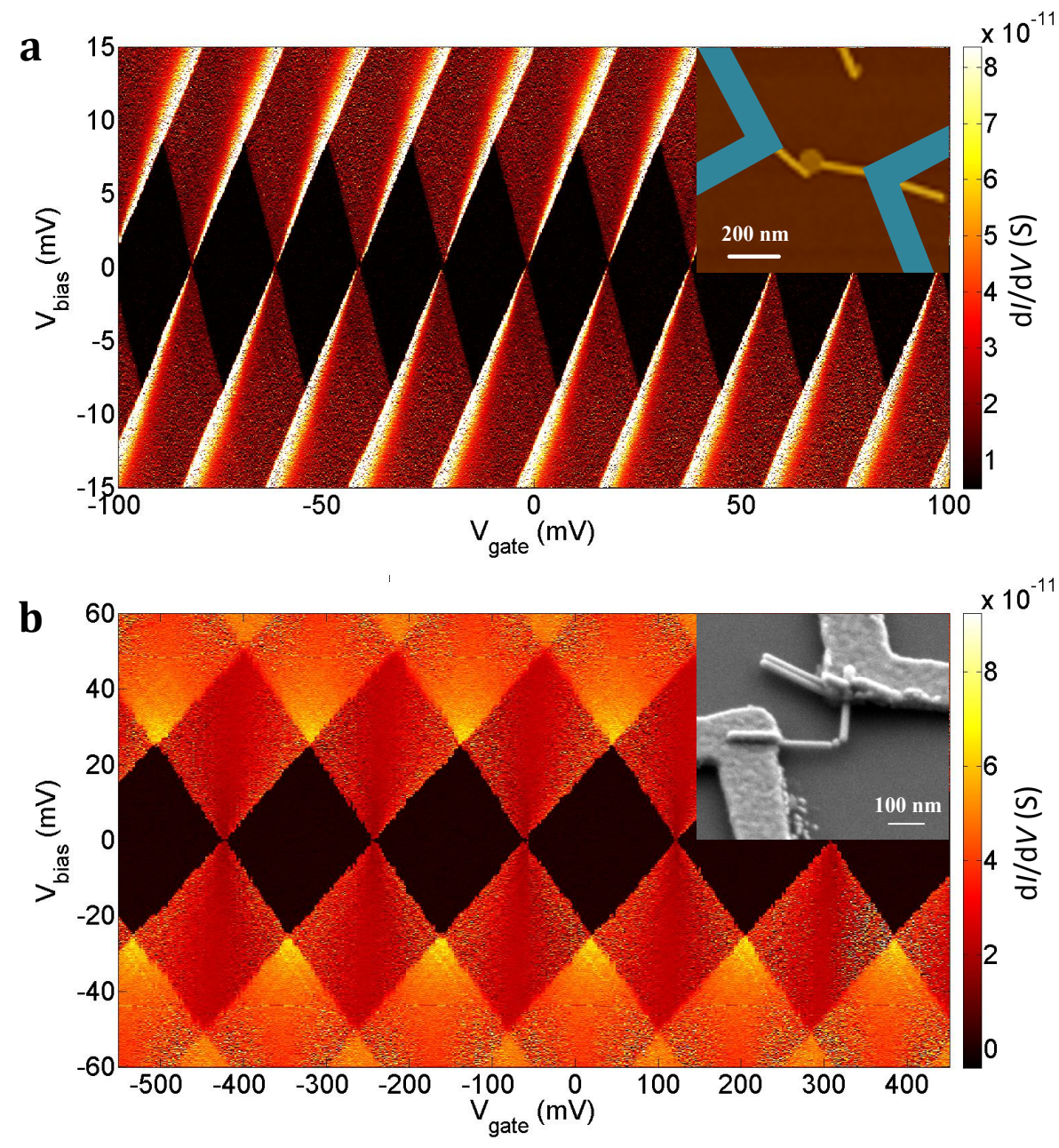

Figure 4.3. Two-dimensional plots of differential conductance as a function of bias voltage $V_{\text {bias }}$ and gate voltage $V_{\text {gate }}$ through SETs fabricated by selective chemical interactions for two different sizes of Coulomb islands: (a) $100 \mathrm{~nm}$ Au flake and (b) $\sim 20 \mathrm{~nm}$ Au NP. Insets demonstrate AFM (a) and SEM (b) images of the corresponding devices. Blue areas inside inset in a show the design of the contacting electrodes prepared by electron beam lithography. ( $T<15 \mathrm{mK}$ ). 
Table 4.1. Calculation of the coupling and interaction parameters of Coulomb islands shown in Fig. 4.3 (see Fig.

1.3 for details).

\begin{tabular}{c|cc}
\hline & $100 \mathrm{~nm}$ Au flake & $20 \mathrm{~nm}$ Au NP \\
\hline$\Delta V_{\text {gate }}$ & $20 \pm 0.3 \mathrm{mV}$ & $146 \pm 2.0 \mathrm{mV}$ \\
$k_{1}$ & 1.038 & 0.408 \\
$k_{2}$ & 0.669 & 0313 \\
$\propto_{\mathrm{g}}=k_{1} k_{2} / k_{1}+k_{2}$ & 0.407 & 0.177 \\
$C_{g}=e / \Delta V_{\text {gate }}$ & $8.01 \cdot 10^{-18} \mathrm{~F}$ & $1.1 \cdot 10^{-18} \mathrm{~F}$ \\
$C_{\mathrm{s}}=C_{\mathrm{g}} / k_{1}$ & $7.72 \cdot 10^{-18} \mathrm{~F}$ & $2.7 \cdot 10^{-18} \mathrm{~F}$ \\
$C_{\mathrm{d}}=C_{\mathrm{g}} / k_{2}-C_{\mathrm{g}}$ & $3.96 \cdot 10^{-18} \mathrm{~F}$ & $2.4 \cdot 10^{-18} \mathrm{~F}$ \\
$C=C_{\mathrm{g}}+C_{\mathrm{s}}+C_{\mathrm{d}}$ & $1.97 \cdot 10^{-17} \mathrm{~F}$ & $6.2 \cdot 10^{-18} \mathrm{~F}$ \\
$\Delta V_{\text {bias }}=\propto_{\mathrm{g}} \Delta V_{\text {gate }}$ & $8.14 \pm 0.1 \mathrm{mV}$ & $25.8 \pm 0.4 \mathrm{mV}$ \\
\hline
\end{tabular}

\subsubsection{Temperature Dependence of the Electron Transport in a Single-Electron Transistor}

Due to the size and nature of the studied Coulomb islands $(\sim 20 \mathrm{~nm}$ in diameter Au NPs) we assume, that the transport through the SETs is a typical classical, or metallic, Coulomb blockade [26, 27]. We studied the temperature dependence of the Coulomb oscillations of SETs. Figure 4.4 shows the temperature dependence of the full-width at the half-maximum (FWHM) of the Coulomb peak in Fig. 4.5 of the device in Fig. 4.6. Here, the top left inset shows the dependence at lower temperatures while the lower right inset shows the Coulomb peak at $T=7 \mathrm{mK}$ (base temperature) fitted with Lorentzian function (tunnelling broadening $h \Gamma$, in red, see Section 1.3) and Eq. 1.9 corresponding to the thermal broadening $k_{\mathrm{B}} T$ in the classical regime (in blue) [28]. From this fitting we can conclude that the peak width is mainly determined by thermal broadening: $h \Gamma \ll k_{\mathrm{B}} T$. (extracted from the fitting corresponding energies are $0.06 \mu \mathrm{eV}$ and $7.45 \mu \mathrm{eV}$, respectively).

The temperature of the electrons in the device (or effective electron temperature $T_{\mathrm{e}}$ ) can differ from the temperature of the dilution refrigerator temperature $T$, mainly due to the noise and pick-up interference (Section 1.8). We can estimate the effective electron temperature from the plot in Fig. 4.4. The slope of the temperature dependence gives a thermal broadening of $4.3 k_{B} T$ (for the $\alpha$-factor equal to 0.095 , extracted from the Coulomb diamonds in Fig. 4.6). This is in good agreement with the theory [28], where the FWHM 
of the Coulomb peak is predicted to be $4.35 k_{\mathrm{B}} T$ for multilevel conductance and supported by experiments in [29]. The FWHM of the peak at $7 \mathrm{mK}$ is equal to $7.45 \mu \mathrm{V}$. This gives us the effective electron temperature $T_{\mathrm{e}}=23 \pm 1 \mathrm{mK}$.

Figure 4.7 shows a typical temperature dependence of the Coulomb oscillations of one of the devices (Fig. 4.3b). In line with theory [29] the Coulomb peak maximum is independent of the temperature as long as $4.3 k_{\mathrm{B}} T \ll e^{2} / C\left(\sim 69.7 \pm 1.1 \mathrm{~K}\right.$, where $e^{2} / C=25.8 \pm 0.4 \mathrm{meV}$ extracted from Fig. 4.6) as predicted for metallic islands (Section 1.3).

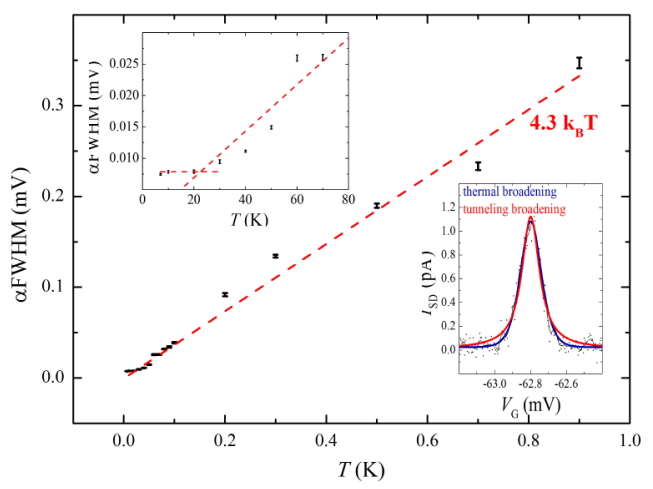

Figure 4.4. Temperature dependence of the full width at half maximum of the Coulomb peak in Fig. 4.5 ( $\alpha$-factor $=0.095$ is extracted from the Coulomb diamonds in Fig. 4.6). Top left inset shows the dependence at lower temperatures. Lower right inset shows Coulomb peak at $T=7 \mathrm{mK}$ fitted with Lorentzian function (tunnelling broadening, in red) and for a thermal broadening (Eq. 1.9, in blue).

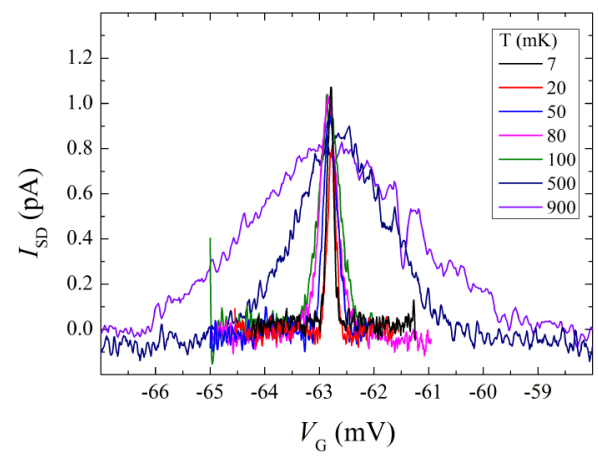

Figure 4.5. Temperature dependence of the Coulomb peak of the singleelectron transistor in Fig. $4.6\left(V_{\text {bias }}=5 \mu \mathrm{V}\right)$. 


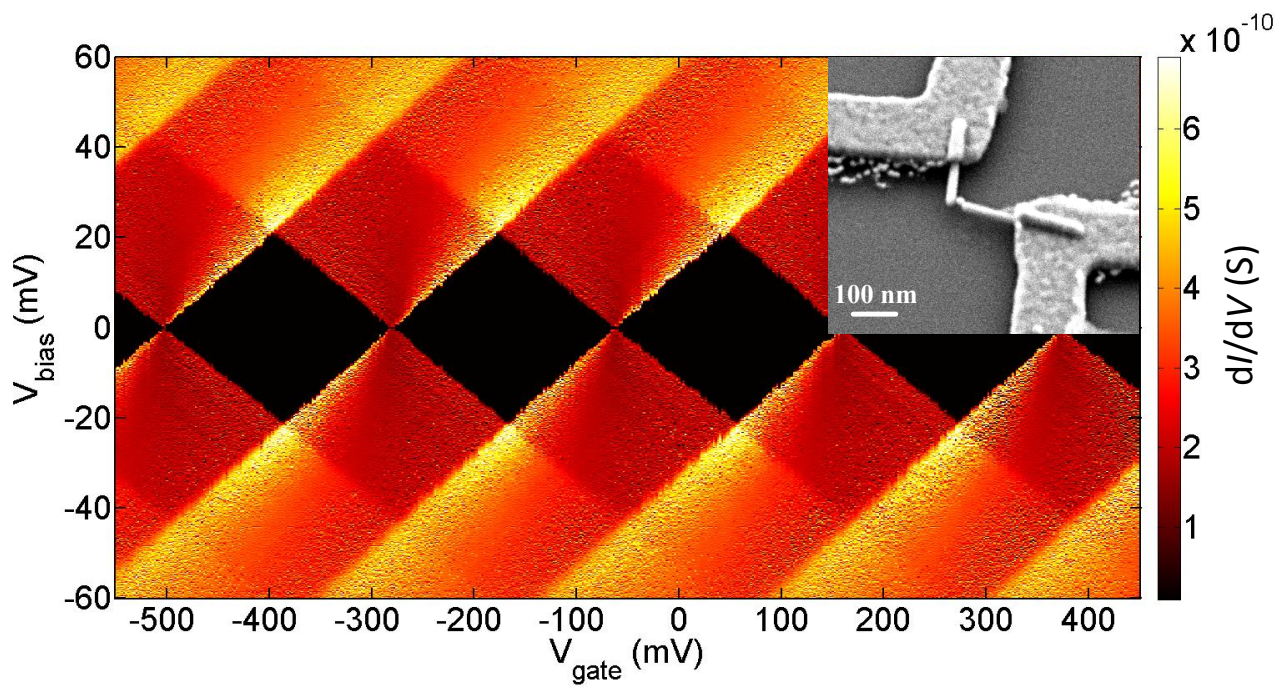

Figure 4.6. Differential conductance as a function of bias voltage $V_{\text {bias }}$ and gate voltage $V_{\text {gate }}$ of a SET fabricated by selective chemical interactions for a $20 \mathrm{~nm}$ Au NP $(T<15 \mathrm{mK})$. Inset: SEM image of the corresponding SET.

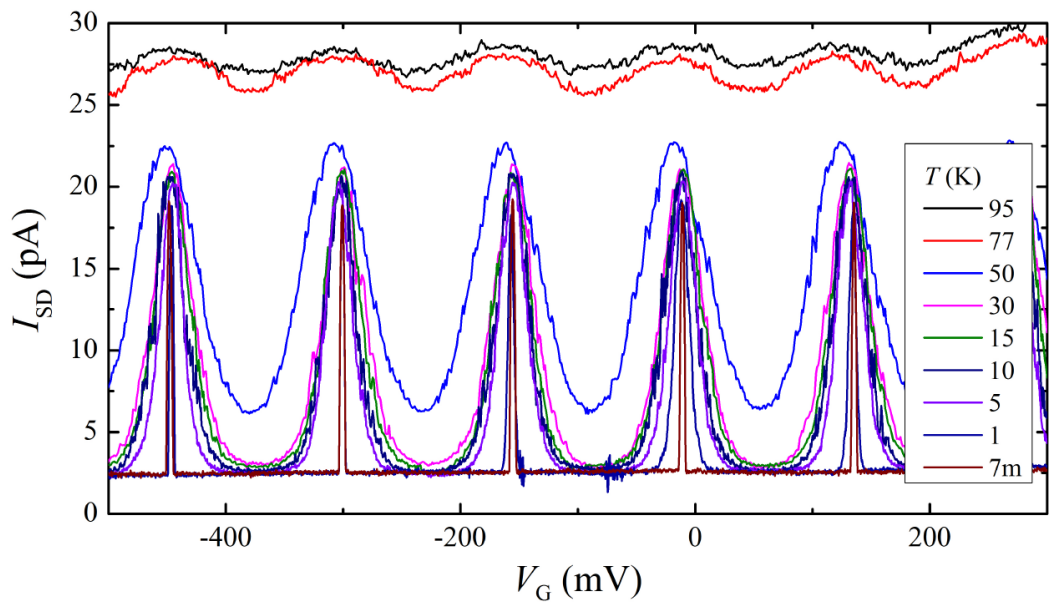

Figure 4.7. Temperature dependence of the Coulomb oscillations of the SET in Fig. 4.3b ( $\left.V_{\text {bias }}=1 \mathrm{mV}\right)$. 


\subsection{Molecular Exchange}

Exchange of the organic ligand molecules around the Au NPs can be performed without changing the structure of the devices (Section 2.5). Here, we show that molecular exchange can change the electronic properties of the SETs made by means of selective chemical interactions. In Figures 4.8-4.9 the electron transport measurements through two different devices is shown before and after replacing the 1,8-octanedithiol shell with $S, S^{\prime}-[1,4-$ Phenylenebis(2,1-ethynediyl-4,1-phenylene)]bis(thioacetate) (OPE3).
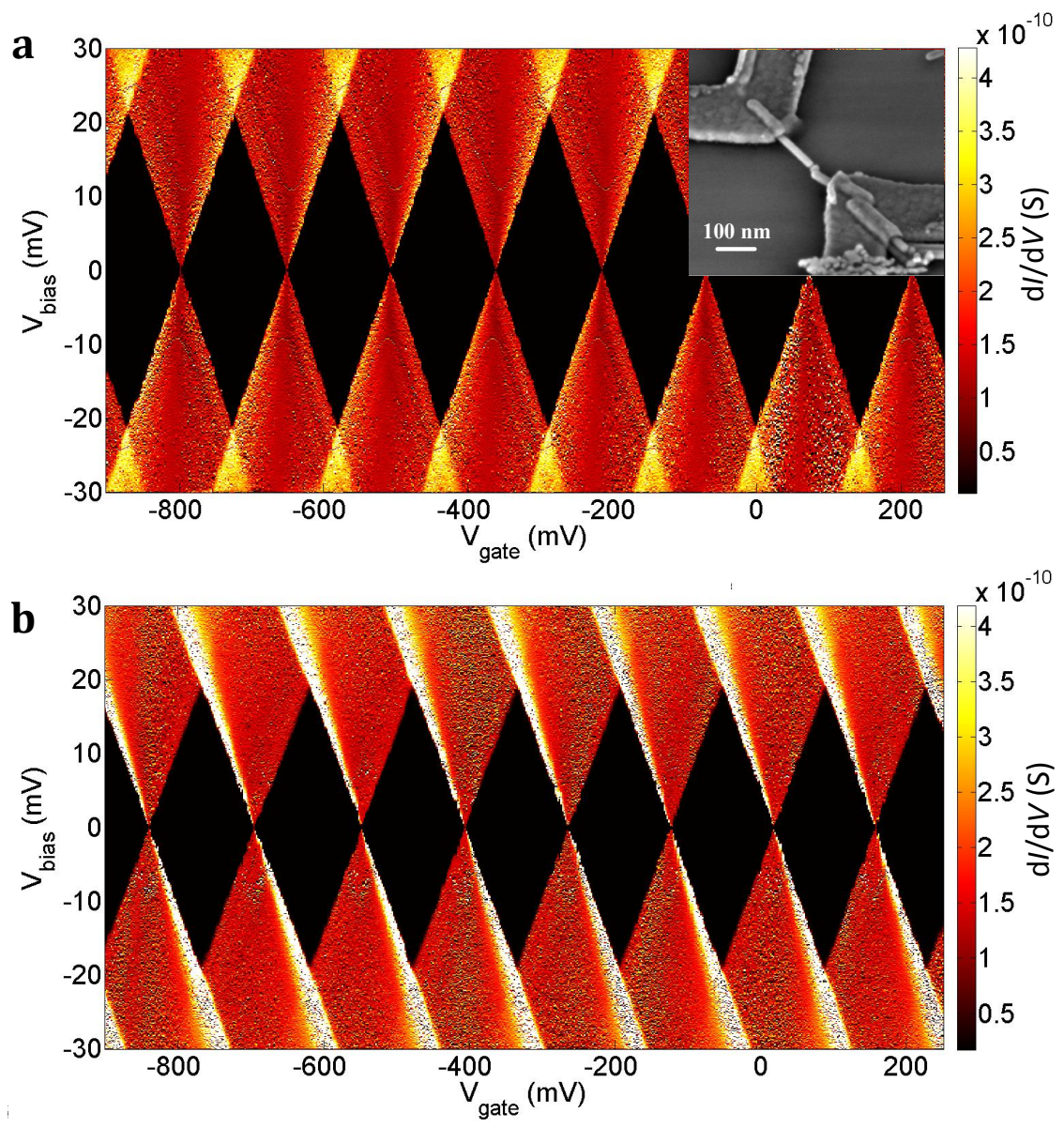

Figure 4.8. Differential conductance as a function of source-drain voltage $V_{\text {bias }}$ and gate voltage $V_{\text {gate }}$ of a SET fabricated by selective chemical interactions containing $20 \mathrm{~nm}$ Au NP ( $T<15 \mathrm{mK}$ ) before (a) and after (b) molecular exchange. Inset in (a) demonstrates SEM image of corresponding device. 


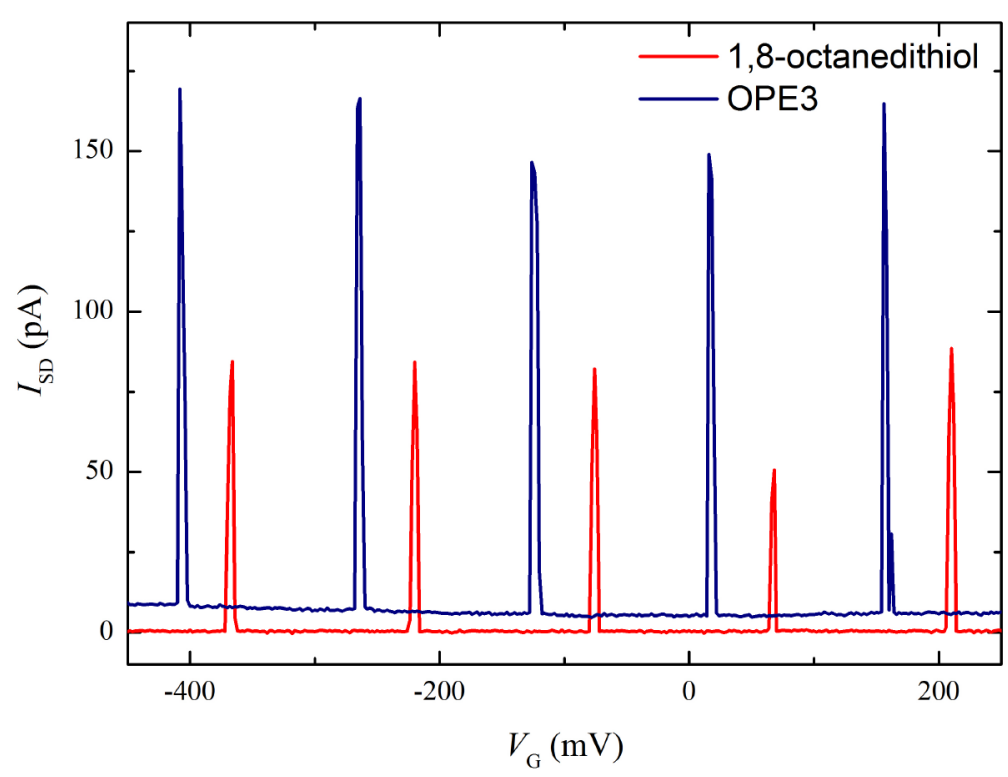

Figure 4.9. Coulomb oscillations through the SET in the inset in Fig. 4.8a showing the increased conductance through the device after molecular exchange of 1,8-dithiol (red) with OPE3 (blue) $\left(V_{\text {bias }}=10 \mu \mathrm{V}, T<15 \mathrm{mK}\right)$..

For the device in Fig. 4.8 the charging energy changes from $20.8 \mathrm{meV}$ (Fig. 4.8a) to $18.4 \mathrm{meV}$ (Fig. 4.8b) (extracted from the differential conductance plots), as well as the coupling to the source/drain obtained more asymmetrical character (what can be explained by the molecular exchange chemical processes which are not studied deeply in present work). Figure 4.9 shows Coulomb oscillations demonstrating that the conductance through the device increased after molecular exchange in agreement with literature. Another device (Fig. 4.10) shows besides decreased charging energy new features (Fig. 4.10b) which might be due to the vibrational modes. (No effect was observed in magnetoresistance measurements).

\section{Conclusions}

We have shown a novel reproducible $(>50 \%)$ method for the fabrication of single-electron transistors via combination of bottom-up self-assembly of nano-objects and top-down lithography. Electrical characterisation at low temperatures revealed a classical Coulomb blockade regime. We investigated Coulomb islands' size and temperature dependences on the SETs' performance. By varying the nature and size of the Coulomb islands (as well as the type of molecular barrier) it is possible to obtain SETs with different 
properties and SETs possibly working at room-temperature. To the best of our knowledge, our specific approach - chemically connecting Au NPs to Au NRs have never been used for the purpose of bottom-up fabricated nanoelectronic devices.
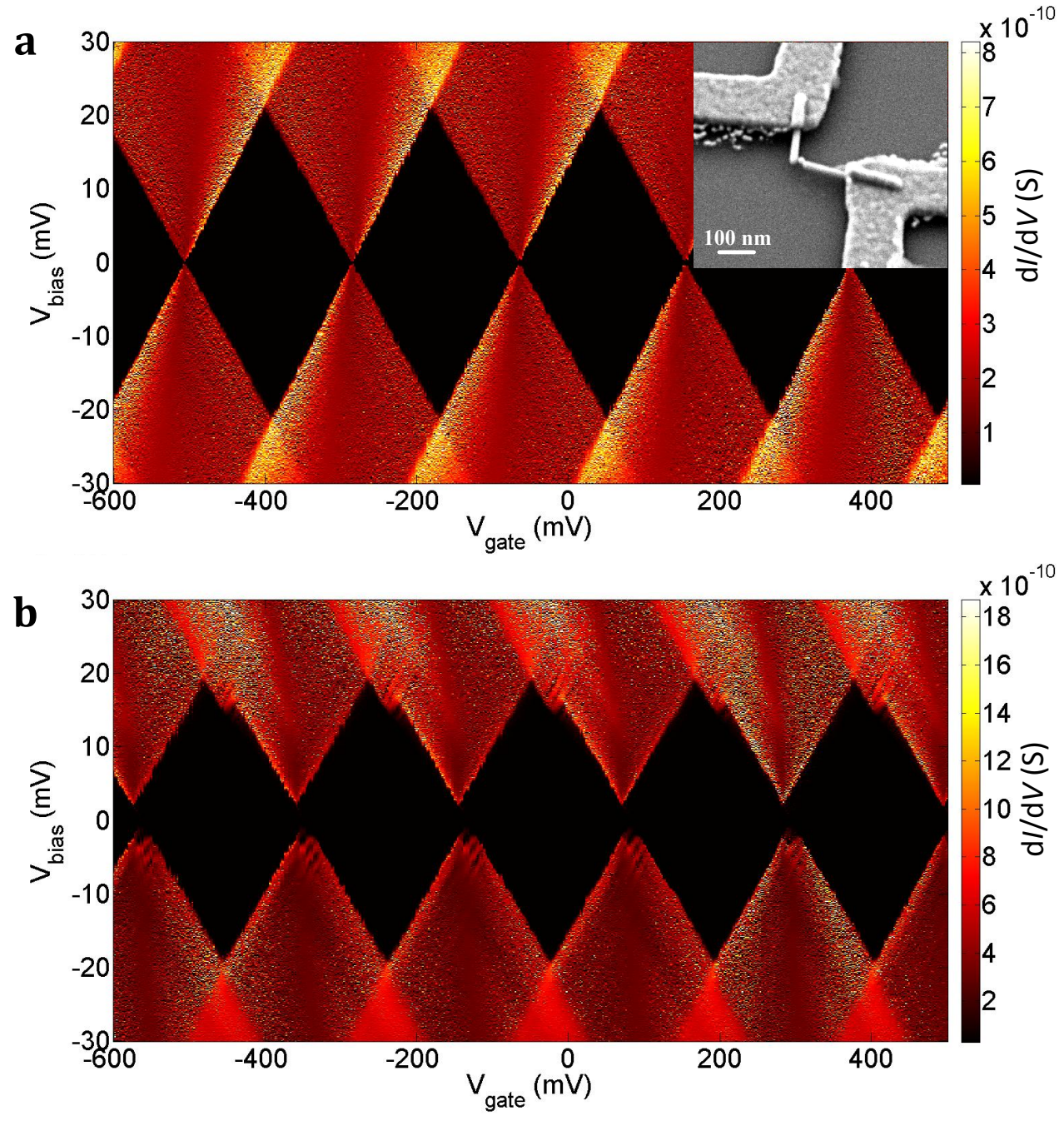

Figure 4.10. Differential conductance as a function of bias voltage $V_{\text {bias }}$ and gate voltage $V_{\text {gate }}$ of a SET fabricated by selective chemical interactions containing $20 \mathrm{~nm}$ Au NP ( $T<15 \mathrm{mK}$ ) before (a) and after (b) molecular exchange. Inset in (a) shows SEM image of the corresponding device. 


\section{REFERENCES}

[1] International Technology Roadmap for Semiconductors. Available: http://www.itrs.net/

[2] M. A. Reed, C. Zhou, C. J. Muller, T. P. Burgin, and J. M. Tour, "Conductance of a Molecular Junction," Science, vol. 278, pp. 252-254, October 10, 19971997.

[3] D. C. Ralph, C. T. Black, and M. Tinkham, "Spectroscopic Measurements of Discrete Electronic States in Single Metal Particles," Physical Review Letters, vol. 74, pp. 3241-3244, 04/17/ 1995.

[4] D. C. Ralph, C. T. Black, and M. Tinkham, "Gate-Voltage Studies of Discrete Electronic States in Aluminum Nanoparticles," Physical Review Letters, vol. 78, pp. 4087-4090, 05/26/ 1997.

[5] J. Park, A. N. Pasupathy, J. I. Goldsmith, C. Chang, Y. Yaish, J. R. Petta, et al., "Coulomb blockade and the Kondo effect in single-atom transistors," Nature, vol. 417, pp. 722-725, 06/13/print 2002.

[6] S. Kubatkin, A. Danilov, M. Hjort, J. Cornil, J. L. Bredas, N. StuhrHansen, et al., "Single-electron transistor of a single organic molecule with access to several redox states," Nature, vol. 425, pp. 698-701, Oct 162003.

[7] B. Xu and N. J. Tao, "Measurement of Single-Molecule Resistance by Repeated Formation of Molecular Junctions," Science, vol. 301, pp. 12211223, August 29, 20032003.

[8] X. D. Cui, A. Primak, X. Zarate, J. Tomfohr, O. F. Sankey, A. L. Moore, et al., "Reproducible measurement of single-molecule conductivity," Science, vol. 294, pp. 571-4, Oct 192001.

[9] E. M. Boon, D. M. Ceres, T. G. Drummond, M. G. Hill, and J. K. Barton, "Mutation detection by electrocatalysis at DNA-modified electrodes," Nature biotechnology, vol. 18, pp. 1096-100, Oct 2000.

[10] K. Hamad-Schifferli, J. J. Schwartz, A. T. Santos, S. Zhang, and J. M. Jacobson, "Remote electronic control of DNA hybridization through inductive coupling to an attached metal nanocrystal antenna," Nature, vol. 415, pp. 152-155, 01/10/print 2002.

[11] T. Dadosh, Y. Gordin, R. Krahne, I. Khivrich, D. Mahalu, V. Frydman, et al., "Measurement of the conductance of single conjugated molecules," Nature, vol. 436, pp. 677-80, Aug 42005.

[12] S. J. van der Molen, J. Liao, T. Kudernac, J. S. Agustsson, L. Bernard, M. Calame, et al., "Light-controlled conductance switching of ordered metalmolecule-metal devices," Nano Letters, vol. 9, pp. 76-80, Jan 2009.

[13] H. Seong, J. Yun, J. H. Jun, K. Cho, and S. Kim, "The transfer of charge carriers photogenerated in $\mathrm{ZnO}$ nanoparticles into a single $\mathrm{ZnO}$ nanowire," Nanotechnology, vol. 20, p. 245201, Jun 172009.

[14] N. I. Kovtyukhova, B. R. Martin, J. K. N. Mbindyo, P. A. Smith, B. Razavi, T. S. Mayer, et al., "Layer-by-Layer Assembly of Rectifying 
Junctions in and on Metal Nanowirest," The Journal of Physical Chemistry B, vol. 105, pp. 8762-8769, 2001/09/01 2001.

[15] A. Gole, C. J. Orendorff, and C. J. Murphy, "Immobilization of Gold Nanorods onto Acid-Terminated Self-Assembled Monolayers via Electrostatic Interactions," Langmuir, vol. 20, pp. 7117-7122, 2004/08/01 2004.

[16] M. A. Correa-Duarte, J. Perez-Juste, A. Sanchez-Iglesias, M. Giersig, and L. M. Liz-Marzan, "Aligning Au nanorods by using carbon nanotubes as templates," Angewandte Chemie (International ed. in English), vol. 44, pp. 4375-8, Jul 112005.

[17] K. G. Thomas, S. Barazzouk, B. I. Ipe, S. T. S. Joseph, and P. V. Kamat, "Uniaxial Plasmon Coupling through Longitudinal Self-Assembly of Gold Nanorods," The Journal of Physical Chemistry B, vol. 108, pp. 1306613068, 2004/09/01 2004.

[18] A. Salant, E. Amitay-Sadovsky, and U. Banin, "Directed Self-Assembly of Gold-Tipped CdSe Nanorods," Journal of the American Chemical Society, vol. 128, pp. 10006-10007, 2006/08/01 2006.

[19] E. C. Cho, S. W. Choi, P. H. Camargo, and Y. Xia, "Thiol-induced assembly of Au nanoparticles into chainlike structures and their fixing by encapsulation in silica shells or gelatin microspheres," Langmuir, vol. 26, pp. 10005-12, Jun 152010.

[20] H. Zhang and D. Wang, "Controlling the growth of charged-nanoparticle chains through interparticle electrostatic repulsion," Angewandte Chemie (International ed. in English), vol. 47, pp. 3984-7, 2008.

[21] I. I. S. Lim, W. Ip, E. Crew, P. N. Njoki, D. Mott, C.-J. Zhong, et al., "Homocysteine-Mediated Reactivity and Assembly of Gold Nanoparticles," Langmuir, vol. 23, pp. 826-833, 2007/01/01 2006.

[22] P. L. Gai and M. A. Harmer, "Surface Atomic Defect Structures and Growth of Gold Nanorods," Nano Letters, vol. 2, pp. 771-774, 2002/07/01 2002.

[23] C. J. Johnson, E. Dujardin, S. A. Davis, C. J. Murphy, and S. Mann, "Growth and form of gold nanorods prepared by seed-mediated, surfactantdirected synthesis," Journal of Materials Chemistry, vol. 12, pp. 17651770, 2002.

[24] J. Pérez-Juste, L. M. Liz-Marzán, S. Carnie, D. Y. C. Chan, and P. Mulvaney, "Electric-Field-Directed Growth of Gold Nanorods in Aqueous Surfactant Solutions," Advanced Functional Materials, vol. 14, pp. 571579, 2004.

[25] P. Horowitz and W. Hill, The Art of Electronics: Cambridge University Press, 1989.

[26] C. Harmans and F. d. T. N. TU Delft, Mesoscopic Physics: An Introduction : TN385 [dictaat Behorende Bij College TN3853]: TU Delft, 2003. 
[27] L. L. Sohn, L. P. Kouwenhoven, and G. Schön, "Mesascopic electron transport," pp. 105-176, 1997.

[28] C. W. J. Beenakker, "Theory of Coulomb-blockade oscillations in the conductance of a quantum dot," Physical Review B, vol. 44, pp. 1646$1656,07 / 15 / 1991$.

[29] E. B. Foxman, U. Meirav, P. L. McEuen, M. A. Kastner, O. Klein, P. A. Belk, et al., "Crossover from single-level to multilevel transport in artificial atoms," Physical Review B, vol. 50, pp. 14193-14199, 11/15/ 1994. 


\section{Chapter 5 ELECTRON TRANSPORT THROUGH A Pair of Metallic Coulomb Islands Coupled in Parallel}

This chapter ${ }^{*}$ is dedicated to the investigation of electron transport measurements through a pair of metallic Coulomb islands in parallel. As Coulomb islands we used Au nanoparticles (NPs) embedded between two Au nanorods (NRs). NPs are decoupled from each other and from NRs by molecular barriers (1.8-octanedithiol monolayer). Low temperature measurements reveal single-electron transport through the device which is strongly dependent on the transport through each island. We observed strong cross-talk between islands, which was systematically studied and analysed. We performed numerical simulations supporting our experimental data.

${ }^{*}$ K.S. Makarenko ${ }^{\dagger}$ et al, in preparation 


\subsection{Double Coulomb Islands Coupled in Parallel}

In the previous chapter formation and studying of the individual metallic islands, acting as a single electron transistors (SETs), was described. The next logical step is to study more than one island. By now, electron charge transport through double Coulomb islands in series has been widely investigated [1-14], while the behaviour of parallel Coulomb islands has been studied less [15-19], and to the best of our knowledge has never been shown before for metallic Coulomb islands. The main focus of this chapter is electron transport through a pair of Coulomb islands coupled in parallel (two $\mathrm{Au}$ nanoparticles (NPs) between two Au nanorods (NRs), Fig. 5.1) created by means of selective chemical interaction (Chapter 4).

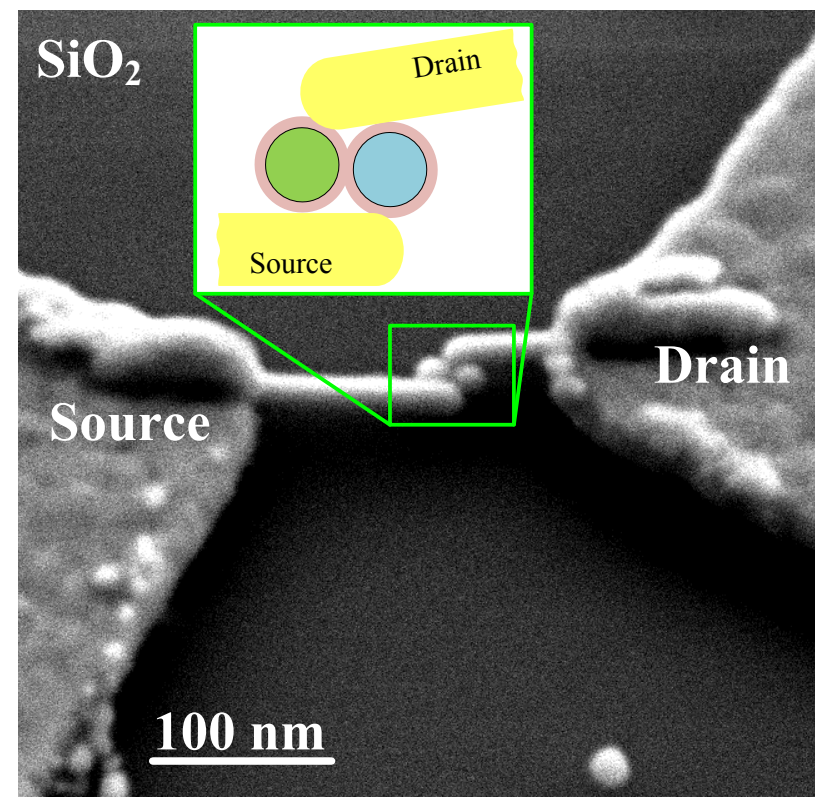

Figure 5.1. SEM image of the two $20 \mathrm{~nm}$ Au NPs enclosed between two Au NRs ( $25 \mathrm{~nm}$ in diameter and $250 \mathrm{~nm}$ long) and schematic of the device.

After the fabrication of the parallel NP system, shown in Fig. 5.1, lowtemperature charge transport measurements $(<15 \mathrm{mK})$ have been performed. Figure 5.2 shows the differential conductance $(\mathrm{d} I / \mathrm{d} V)$ of this coupled SET system as a function of the source-drain voltage $\left(V_{\text {bias }}\right)$ and the back gate voltage $\left(V_{\text {gate }}\right)$ applied to the $\mathrm{p}++\mathrm{Si}$ substrate, acting as a back gate underneath $35 \mathrm{~nm} \mathrm{SiO}$. 


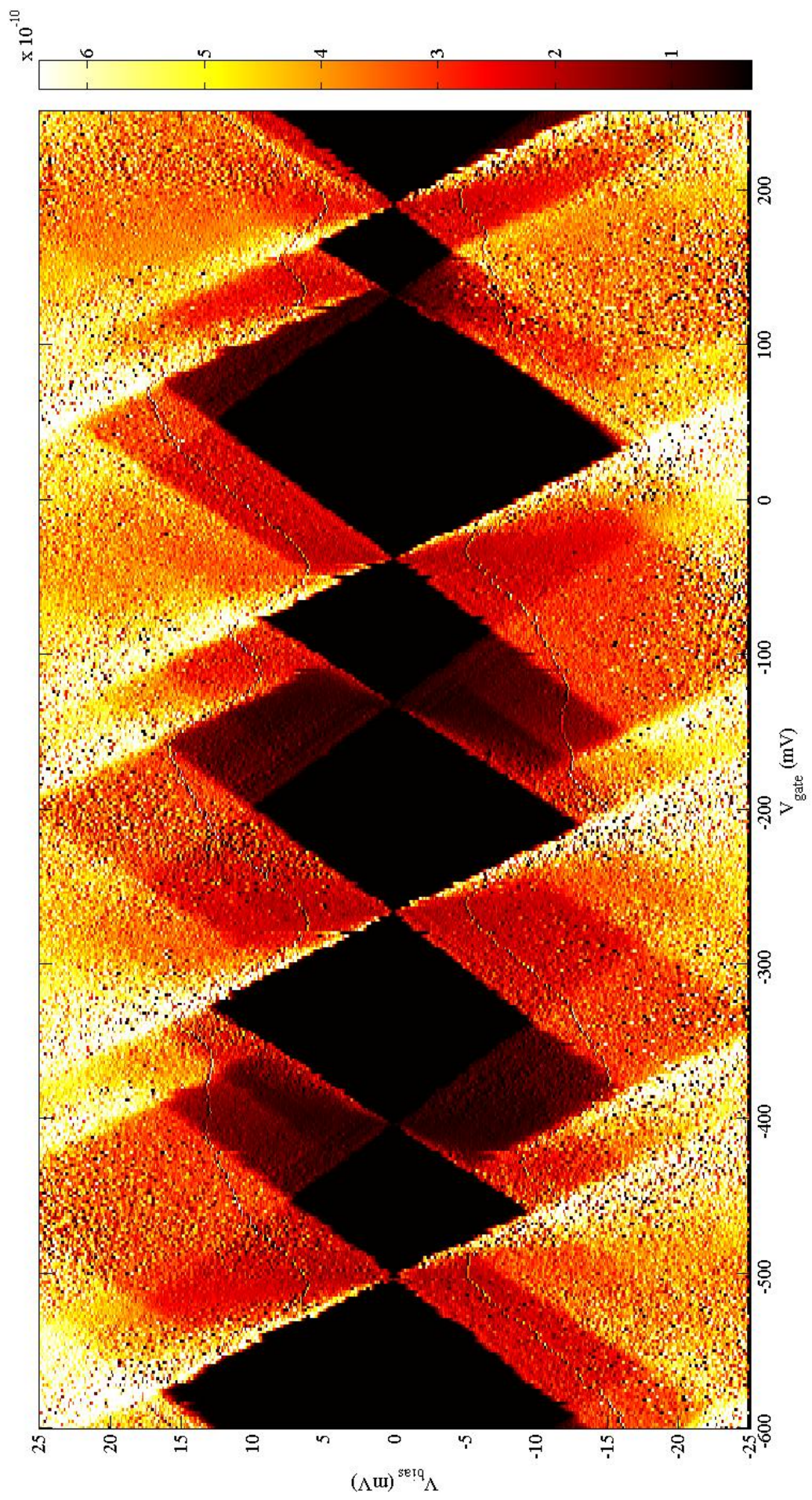

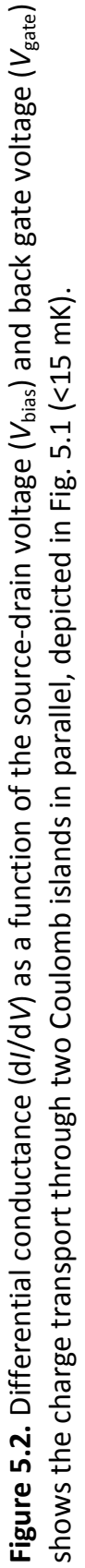




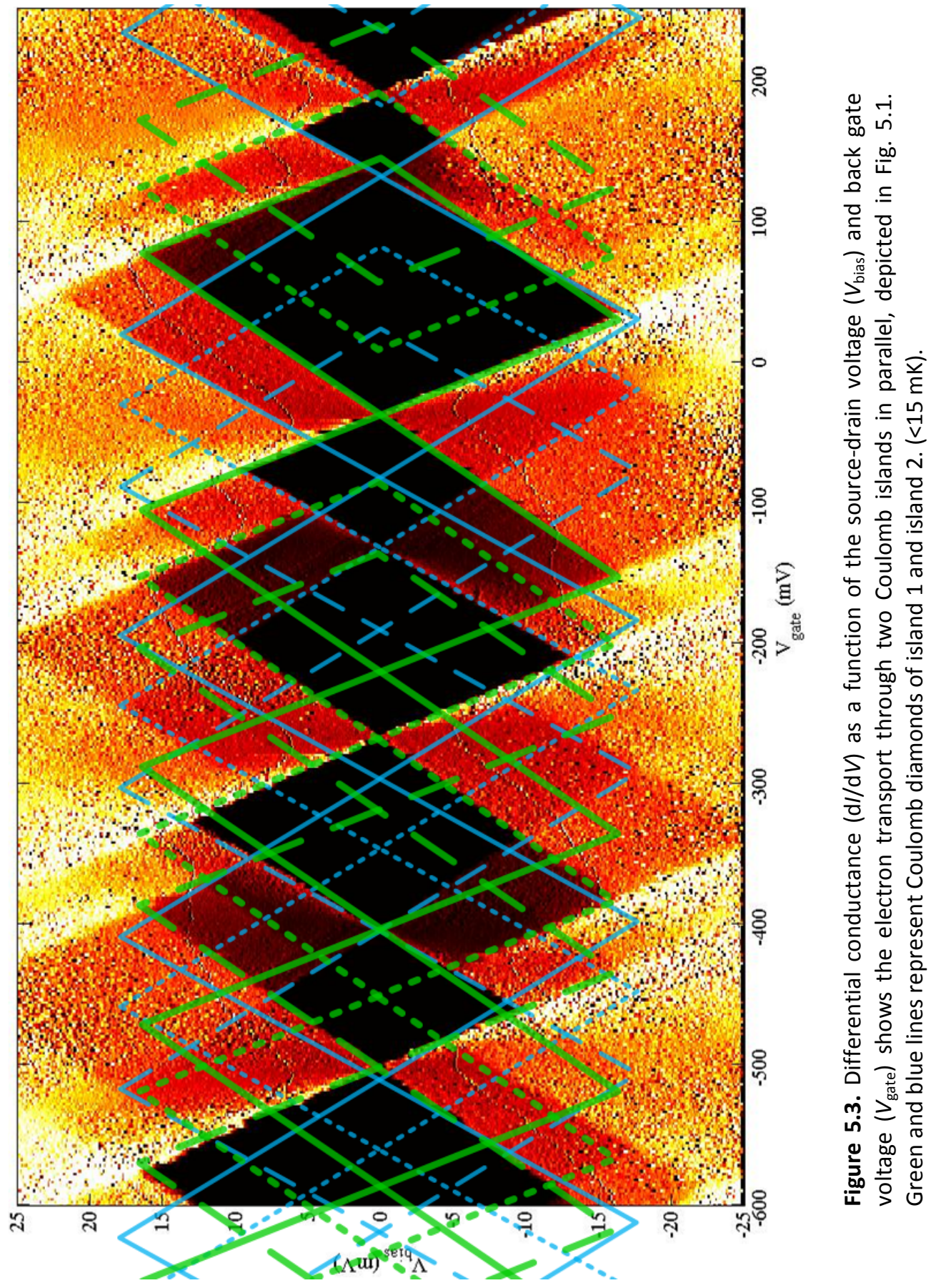




\subsection{Electron Transport through a Pair of Coulomb Islands Coupled in Parallel}

A pair of Coulomb islands coupled in parallel in Fig. 5.1 can be represented as a network of tunnel resistors and capacitors (Fig. 5.4). The number of electrons on island 1 and 2 is $N$ and $M$, respectively. Both islands are capacitively coupled to $V_{\text {gate }}$ through capacitors $C_{\mathrm{g} 1(2)}$ and to the source $(s)$ and drain $(d)$ contacts through tunnel resistors $R_{\mathrm{s}(\mathrm{d}) 1(2)}$ and capacitors $C_{\mathrm{s}(\mathrm{d}) 1(2) \text {. }}$ Islands are also coupled to each other through tunnel resistor $R_{\mathrm{m}}$ and capacitor $C_{\mathrm{m}}$.
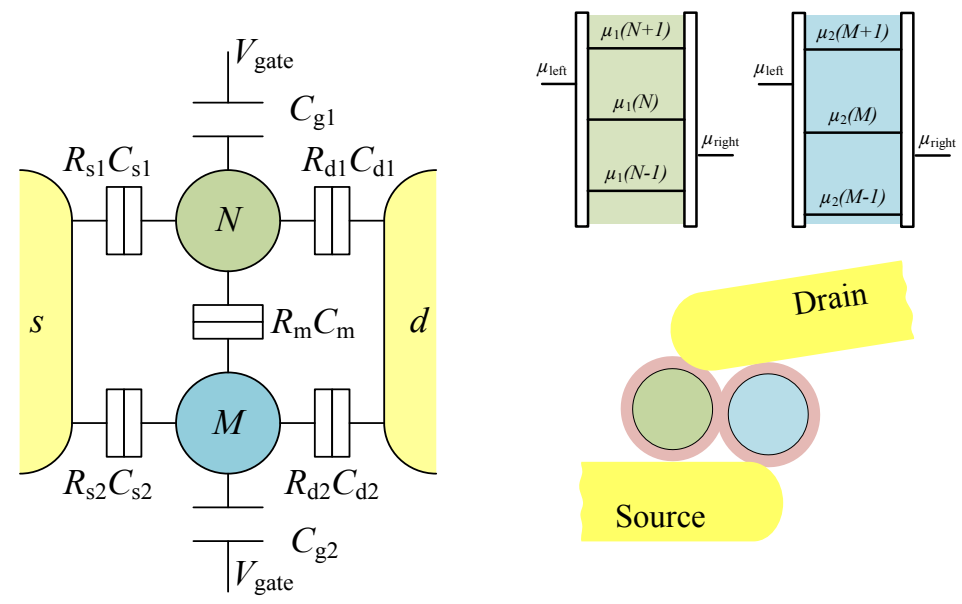

Figure 5.4. Schematic of two Coulomb islands in parallel (left) and potential diagrams showing electron transport through parallel islands (right).

In the absence of the tunnel barrier with $R_{\mathrm{m}} C_{\mathrm{m}}$ between the two $\mathrm{Au}$ NPs, the electron transport through one island is independent of the other one, which leads to the superposition of two independent Coulomb diamonds features in the stability plot $\left(\mathrm{d} I / \mathrm{d} V=\mathrm{d} I_{1} / \mathrm{d} V+\mathrm{d} I_{2} / \mathrm{d} V\right.$ vs. $V_{\text {gate }}$ and $\left.V_{\text {bias }}\right)$. However, the cross-talk between charges occupying the two islands leads to the complex electron behaviour in Fig. 5.2, and the influence of one electron coming on/off one of the island on the transport of another one can be described as a potential difference [1]:

$$
\Delta V=\frac{e}{C_{\mathrm{m}}}\left(\frac{1}{\frac{C_{1} C_{2}}{C_{\mathrm{m}}^{2}}-1}\right)
$$


where $C_{1(2)}$ is the sum of the capacitances attached to the Coulomb island $1(2)$.

Charge transport through one island strongly depends on the other one. Corresponding stability diagrams of both islands are depicted in Fig. 5.3 (same as Fig. 5.2 but now with Coulomb diamonds of respective islands indicated). Here, the solid green lines (-) correspond to the Coulomb diamonds of island 1 when island 2 has a fixed number of electrons $M$. When island 2 loses/gains one electron $(M-1 / M+1)$, green diamonds shift to the left/right (.........) from the original ones by $\Delta V$ (Appendix). Losing/gaining two electrons $(M-2 / M+2)$ is leading to the shift of blue diamonds by $2 \Delta V(---)$, and so on. The same happens with the Coulomb diamonds of island 2 when electrons jumps on/off island 1 (Appendix A).

To clarify the electron transport through the double Coulomb islands in parallel, electrochemical diagrams can be used. Figure 5.5 explains the electron behaviour at zero $V_{\text {bias }}$ for $-300<V_{\text {gate }}<10 \mathrm{mV}$. Position (0) depicts a situation when no $V_{\text {gate }}$ is applied, and islands 1 and 2 have $N$ and $M$ electrons, respectively. When a negative $V_{\text {gate }}$ is applied, the energy levels of the Coulomb islands are pulled up. At position (1) there is an available level $\mu_{1}(N, M)$ on island 1 , and after crossing this position island 1 has $N-1$ electrons. This causes the lowering of the level of island $2 \mu_{2}(N, M)$ to $\mu_{2}(N-1, M)$. As a consequence, at position (2), when the applied $V_{\text {gate }}$ aligns $\mu_{2}(N, M)$ to the source/drain, the transport through this level is not possible anymore because the level is not relevant after losing an electron by island 1. At position (3) the transport through island 1 is forbidden since electrons cannot go through the aligned level $\mu_{1}(N, M-1)$ due to the fact that island 1 and island 2 have $N-1$ and $M$ electrons, respectively. At position (4) electron transport is possible through island 2 through level $\mu_{2}(N-1, M)$.

Current through one tunnel barrier is determined by [20]:

$$
I=\frac{e}{\tau}=e \Gamma
$$

where $e$ is elementary charge, $\tau$ is the dwell time and $\Gamma$ is tunnelling rate. In case of the one Coulomb island there are two barriers in series, so the current is given by:

$$
I=\frac{e}{\tau_{\mathrm{i}}^{\mathrm{S}}+\tau_{\mathrm{i}}^{\mathrm{d}}}=e \frac{\Gamma_{\mathrm{i}}^{\mathrm{S}} \Gamma_{\mathrm{i}}^{\mathrm{d}}}{\Gamma_{\mathrm{i}}^{\mathrm{s}}+\Gamma_{\mathrm{i}}^{\mathrm{d}}}
$$

where $\tau_{\mathrm{i}}^{\mathrm{s}}$ and $\tau_{\mathrm{i}}^{\mathrm{d}}$ are the dwell times, and $\Gamma_{\mathrm{i}}^{\mathrm{s}}$ and $\Gamma_{\mathrm{i}}^{\mathrm{d}}$ are tunnelling rates of the source and drain barriers, respectively; $i$ is the number of the island (1 or 2).

In Figs. 5.2-5.3, brighter colours correspond to the higher current through islands, which means that the total current through island 2 is 
determined by the tunnelling rate of molecular barrier between island 2 and the drain electrode, while charge transport through island 1 is governed by the tunnelling rate of the molecular barrier between island 1 and the source electrode. In other words, island 1 is stronger coupled to the drain, while island 2 to the source electrode (see schematic in Fig. 5.4):

$$
\begin{aligned}
& I_{1}=e \frac{\Gamma_{1}^{\mathrm{s}} \Gamma_{1}^{\mathrm{d}}}{\Gamma_{1}^{\mathrm{s}}+\Gamma_{1}^{\mathrm{d}}} \approx e \frac{\Gamma_{1}^{\mathrm{s}} \Gamma_{1}^{\mathrm{d}}}{\Gamma_{1}^{\mathrm{d}}} \approx e \Gamma_{1}^{\mathrm{s}},\left(\Gamma_{1}^{\mathrm{s}} \gg \Gamma_{1}^{\mathrm{d}}\right) \\
& I_{2}=e \frac{\Gamma_{2}^{\mathrm{s}} \Gamma_{2}^{\mathrm{d}}}{\Gamma_{2}^{\mathrm{s}}+\Gamma_{2}^{\mathrm{d}}} \approx e \frac{\Gamma_{2}^{\mathrm{s}} \Gamma_{2}^{\mathrm{d}}}{\Gamma_{2}^{\mathrm{s}}} \approx e \Gamma_{2}^{\mathrm{d}},\left(\Gamma_{2}^{\mathrm{s}} \ll \Gamma_{2}^{\mathrm{d}}\right)
\end{aligned}
$$

a

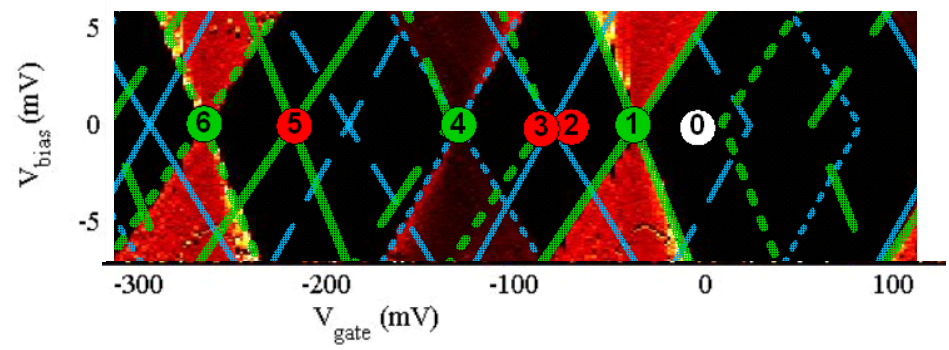

b
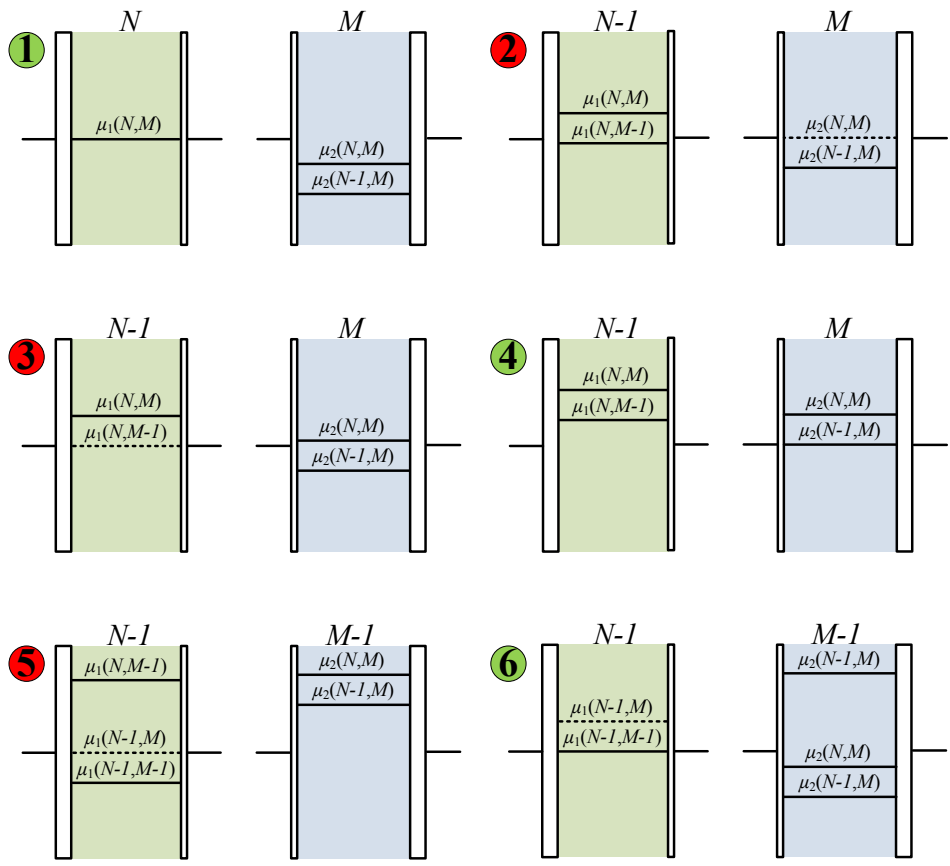

Figure 5.5. a, Zoom-in of the stability diagram of Fig. 5.3, focused at zero $V_{\text {bias. }} \cdot \mathbf{b}$, Schematic diagrams of the electrochemical potentials $\mu_{1(2)}(N, M)$ in islands and leads for the positions (1)-(6) depicted in a. 
Using the schematic stability diagram in Fig. 1.3, it is possible to calculate the coupling and interaction parameters of the two Coulomb islands depicted in Fig. 5.1 (Table 5.1). Based on Table 5.1 and Eq. (5.4)-(5.5) a general schematic electrochemical-potential diagram of the two parallel Coulomb islands can be drawn (Fig. 5.6) and is used to explain the current steps in the experimental data in Fig. 5.2.

Table 5.1. Calculation of the coupling and interaction parameters of a pair of Coulomb islands in parallel from Fig. 5.1

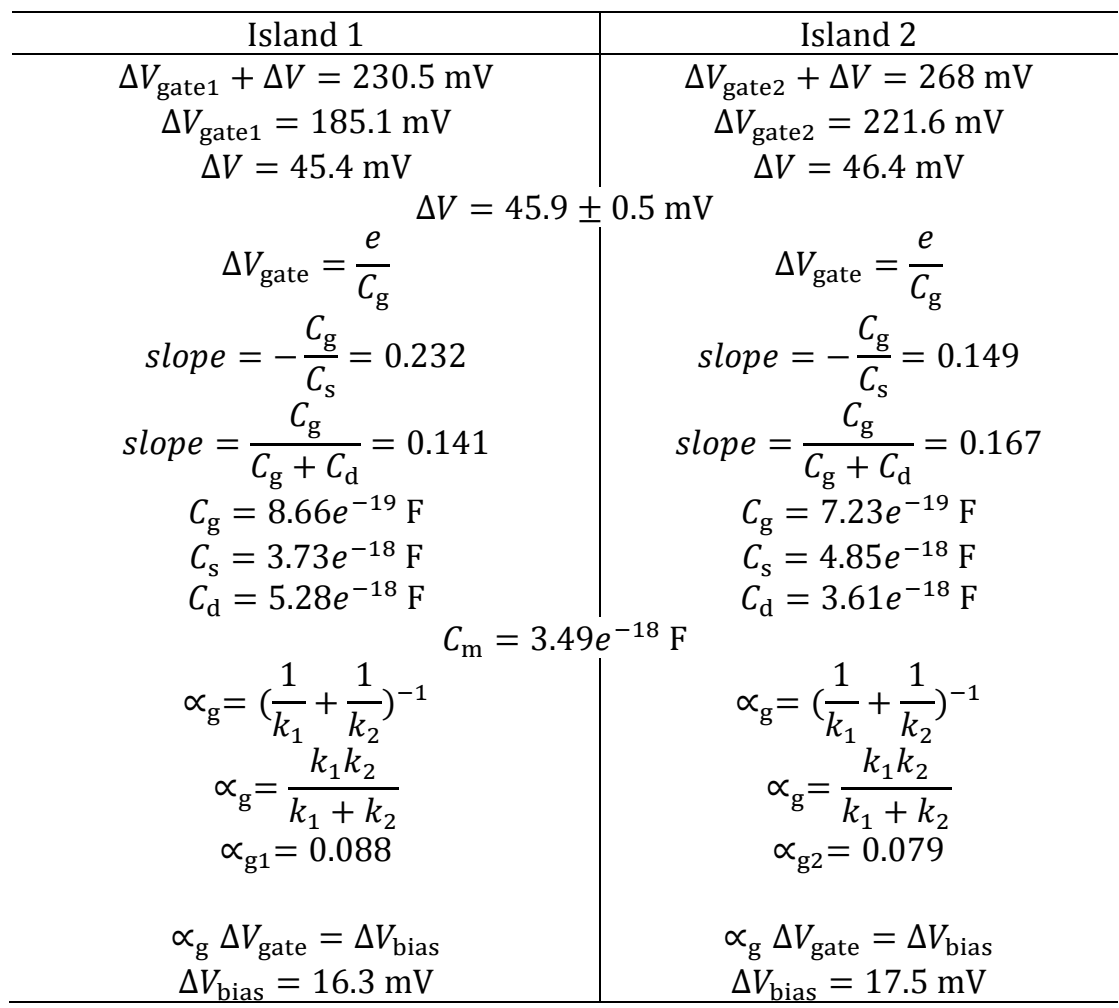




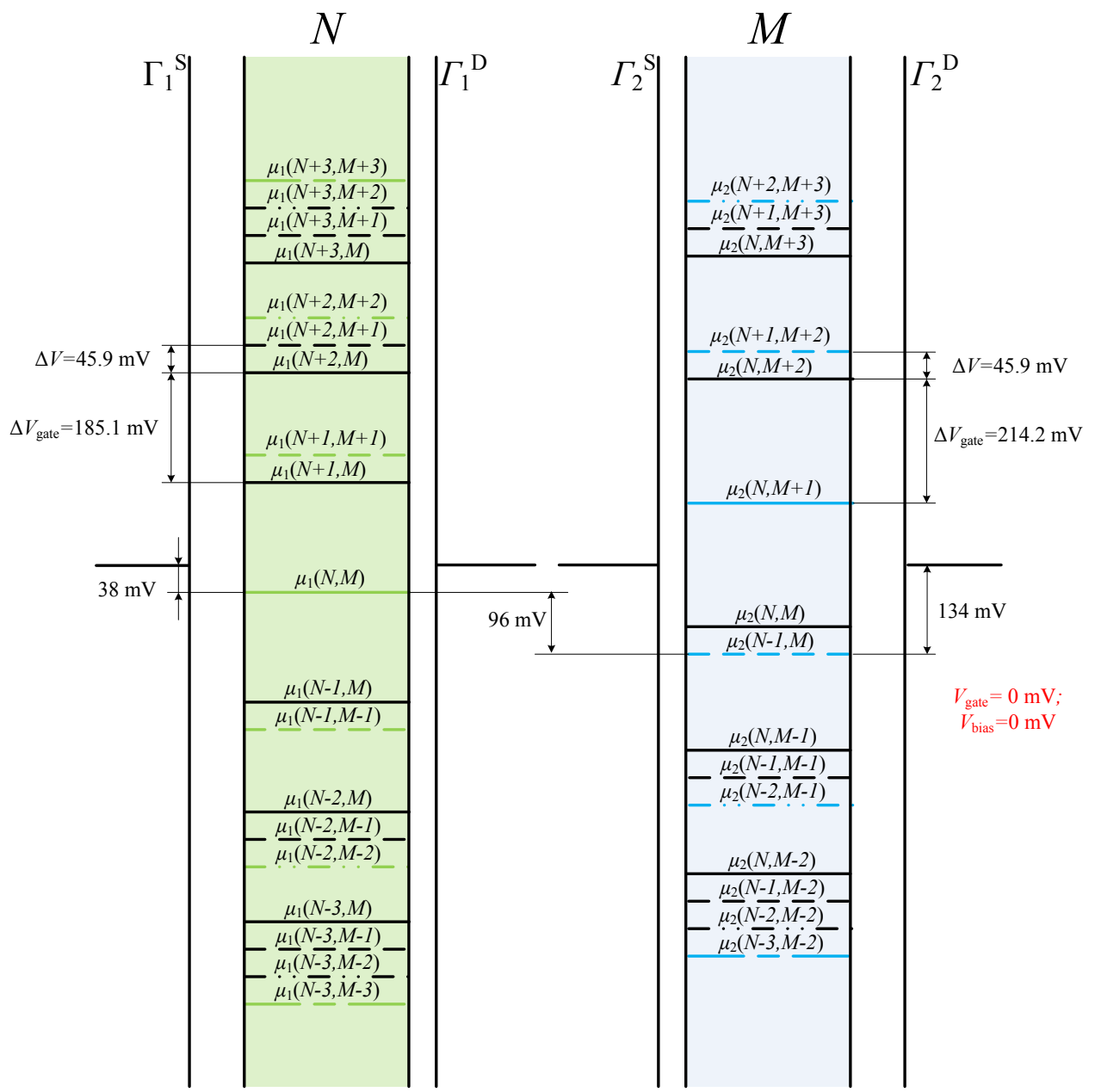

Figure 5.6. Schematic diagram of electrochemical potentials of a pair of Coulomb islands in parallel at zero $V_{\text {bias }}$ and zero $V_{\text {gate }}$ which can be used to explain any point of the stability diagram in Fig. 5.2.

\subsection{Simulation Results}

As stated before, when the tunnel barrier with $R_{\mathrm{m}} C_{\mathrm{m}}$ between the two Au NPs is absent, electron transport through one island is independent of the other one, while the presence of a cross-talk between them changes the electron transport dramatically. We show this by numerical calculations, based on the coupling and interaction parameters extracted from the experimental data 
(Fig. 5.2, Table 5.1), using the commercial software SIMON 2.0. A pair of Coulomb islands which are coupled in parallel can be represented as a network of tunnel resistors and capacitors (Fig. 5.4). Simulation results of the electron behaviour through two Coulomb islands in parallel, when islands are not capacitively coupled to each other (Fig. 5.7a), is represented in Fig. 5.8. Here, two sets of diamonds corresponding to two Coulomb islands are independent of each other showing that in the absence of the tunnel barrier between islands the electron transport through one island does not influence the other one. However, Fig. 5.9 shows a remarkable change in the electron transport behaviour when a strong capacitive coupling between Coulomb islands is present (Fig. 5.7b).
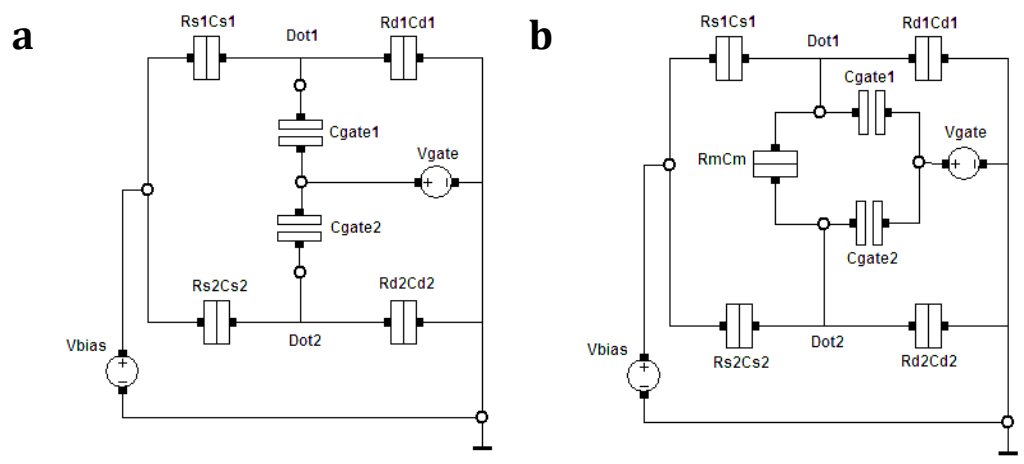

Figure 5.7. Network of tunnel resistors and capacitors representing a pair of Coulomb islands in parallel without (a) and with (b) finite interisland coupling .

\section{Conclusions}

Measurements on double quantum islands in parallel reveal evidence of the interaction between two islands which results in a variety of features in the charge transport spectra. Data analysis and numerical simulations shown that capacitive inter-island coupling leads to the shifting of the regular Coulomb diamonds. The results, represented in this chapter, contribute to the understanding the charge transport behaviour through the hybrid inorganicorganic single-electron transport device containing a pair of interacting Coulomb islands in parallel. 


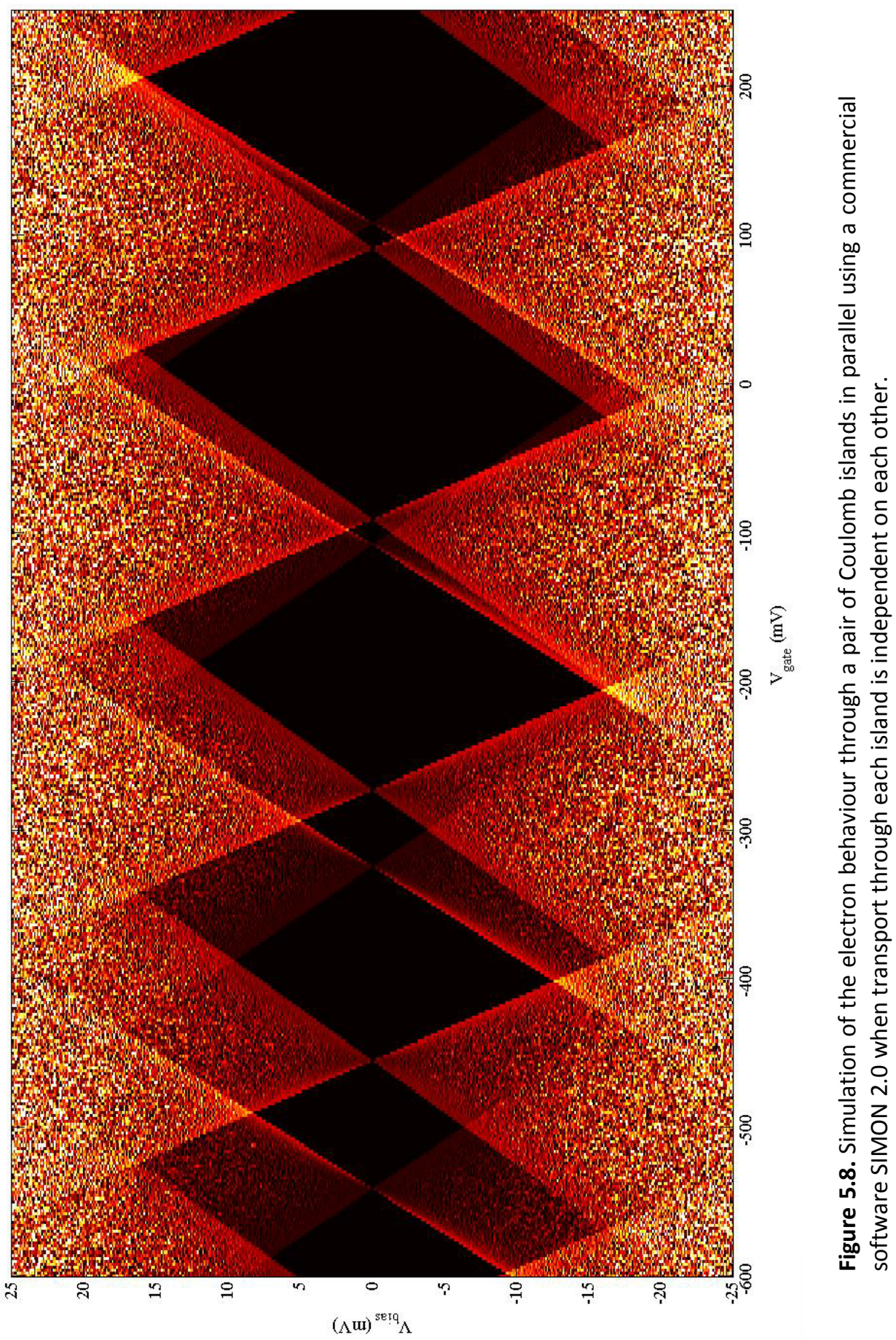




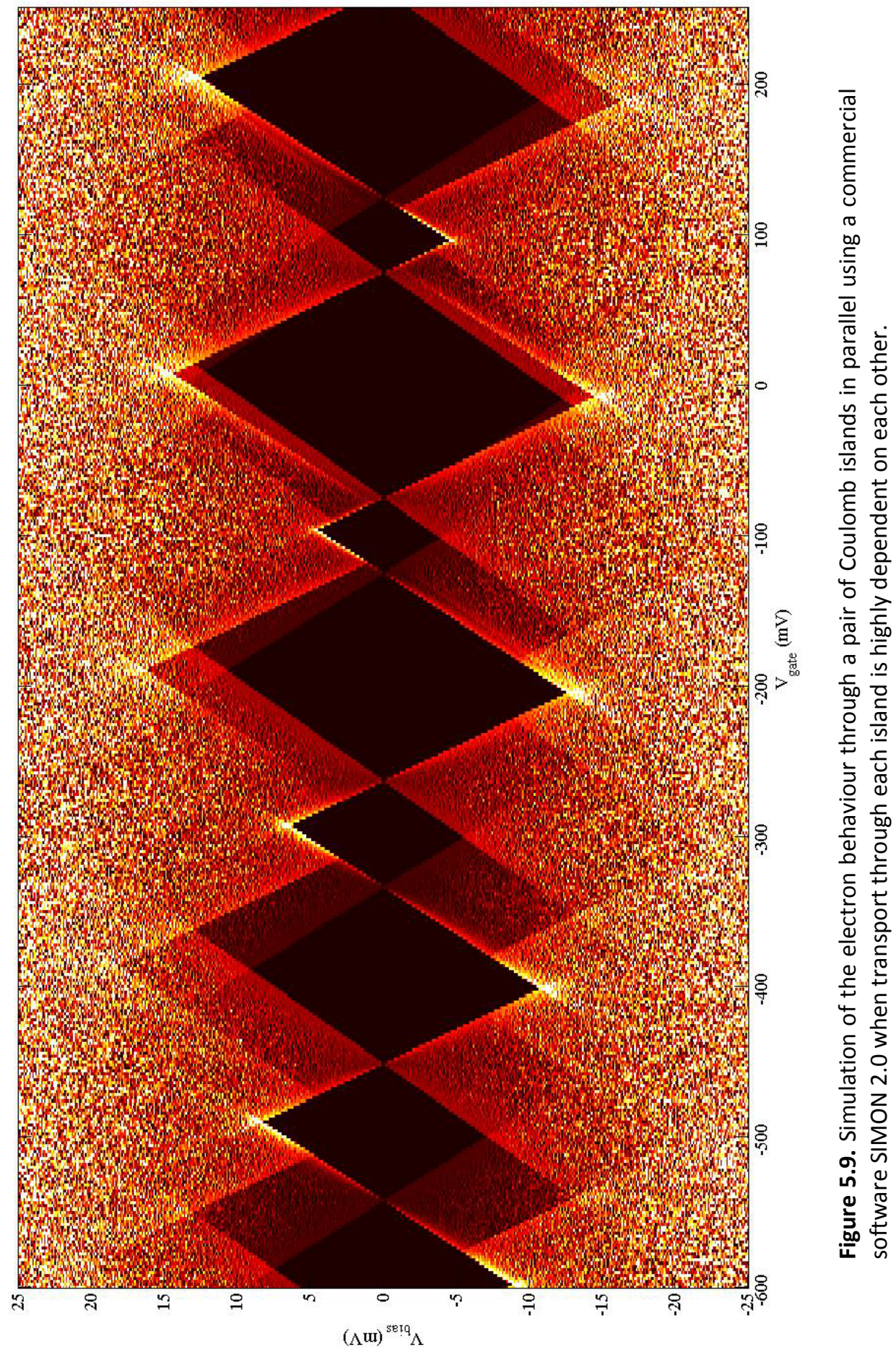




\section{REFERENCES}

[1] W. G. van der Wiel, S. De Franceschi, J. M. Elzerman, T. Fujisawa, S. Tarucha, and L. P. Kouwenhoven, "Electron transport through double quantum dots," Reviews of Modern Physics, vol. 75, pp. 1-22, 12/17/ 2002.

[2] R. H. Blick, R. J. Haug, J. Weis, D. Pfannkuche, K. v. Klitzing, and K. Eberl, "Single-electron tunneling through a double quantum dot: The artificial molecule," Physical Review B, vol. 53, pp. 7899-7902, 03/15/ 1996.

[3] R. H. Blick, D. Pfannkuche, R. J. Haug, K. v. Klitzing, and K. Eberl, "Formation of a Coherent Mode in a Double Quantum Dot," Physical Review Letters, vol. 80, pp. 4032-4035, 05/04/ 1998.

[4] D. Dixon, L. P. Kouwenhoven, P. L. McEuen, Y. Nagamune, J. Motohisa, and H. Sakaki, "Linear and non-linear transport through coupled quantum dots," Surface Science, vol. 361-362, pp. 636-639, 7/20/ 1996.

[5] T. Fujisawa, T. H. Oosterkamp, W. G. van der Wiel, B. W. Broer, R. Aguado, S. Tarucha, et al., "Spontaneous Emission Spectrum in Double Quantum Dot Devices," Science, vol. 282, pp. 932-935, October 30, 1998 1998.

[6] T. Fujisawa and S. Tarucha, "Photon assisted tunnelling in single and coupled quantum dot systems," Superlattices and Microstructures, vol. 21, pp. 247-254, 3// 1997.

[7] H. Jeong, S. P. Mason, A. L. Barabasi, and Z. N. Oltvai, "Lethality and centrality in protein networks," Nature, vol. 411, pp. 41-42, 05/03/print 2001 .

[8] L. W. Molenkamp, K. Flensberg, and M. Kemerink, "Scaling of the Coulomb Energy Due to Quantum Fluctuations in the Charge on a Quantum Dot," Physical Review Letters, vol. 75, pp. 4282-4285, 12/04/ 1995.

[9] L. W. Molenkamp and M. Kemerink, "Stochastic Coulomb blockade and scaling of charging energy in a double quantum dot system," Superlattices and Microstructures, vol. 16, pp. 275-278, // 1994.

[10] T. H. Oosterkamp, T. Fujisawa, W. G. van der Wiel, K. Ishibashi, R. V. Hijman, S. Tarucha, et al., "Microwave spectroscopy of a quantum-dot molecule," Nature, vol. 395, pp. 873-876, 10/29/print 1998.

[11] Toshimasa Fujisawa and Seigo Tarucha, "Multiple Photon Assisted Tunneling between Two Coupled Quantum Dots," Japanese Journal of Applied Physics, vol. 36, p. 4000, 1997.

[12] N. C. Van Der Vaart, L. P. Kouwenhoven, S. F. Godijn, Y. V. Nazarov, C. J. P. M. Harmans, J. E. Mooij, et al., "Two coupled quantum dots with a continuous density of states," Superlattices and Microstructures, vol. 24, pp. 435-441, 12// 1998. 
[13] F. R. Waugh, M. J. Berry, C. H. Crouch, C. Livermore, D. J. Mar, R. M. Westervelt, et al., "Measuring interactions between tunnel-coupled quantum dots," Physical Review B, vol. 53, pp. 1413-1420, 01/15/ 1996.

[14] F. R. Waugh, M. J. Berry, D. J. Mar, R. M. Westervelt, K. L. Campman, and A. C. Gossard, "Single-Electron Charging in Double and Triple Quantum Dots with Tunable Coupling," Physical Review Letters, vol. 75, pp. 705-708, 07/24/ 1995.

[15] A. S. Adourian, C. Livermore, R. M. Westervelt, K. L. Campman, and A. C. Gossard, "Single electron charging in parallel coupled quantum dots," Superlattices and Microstructures, vol. 20, pp. 411-417, 10// 1996.

[16] A. S. Adourian, C. Livermore, R. M. Westervelt, K. L. Campman, and A. C. Gossard, "Evolution of Coulomb blockade spectra in parallel coupled quantum dots," Applied Physics Letters, vol. 75, pp. 424-426, 1999.

[17] M. Arai, S. Masubuchi, and T. Machida, "Single-electron switching effect in graphene parallel-coupled double quantum dots," Journal of Physics: Conference Series, vol. 334, p. 012041, 2011.

[18] K. Goß, M. Leijnse, S. Smerat, M. R. Wegewijs, C. M. Schneider, and C. Meyer, "Parallel carbon nanotube quantum dots and their interactions," Physical Review B, vol. 87, p. 035424, 01/23/ 2013.

[19] F. Hofmann, T. Heinzel, D. A. Wharam, J. P. Kotthaus, G. Böhm, W. Klein, et al., "Single electron switching in a parallel quantum dot," Physical Review B, vol. 51, pp. 13872-13875, 05/15/ 1995.

[20] H. van Houten, C. W. J. Beenakker, and A. A. M. Staring, "CoulombBlockade Oscillations in Semiconductor Nanostructures," ed, 2005. 


\section{Chapter 6 DyNAMICAL Nonlocality IN A DIFFUSIVE QUANTUM INTERFEROMETER}

In this chapter ${ }^{*}$ a Au Aharonov-Bohm (AB) interferometer embedded between two pairs of Au leads (one for the current leads and one for the voltage probes) is employed for a consistent study of the quantum dynamic nonlocality in diffusive system. Here, we perform investigation of the nonlocal signal, and demonstrate that, despite the diffusive motion of electrons, nonlocal quantum correlations can also be observed and controlled in normal metals and be even more pronounced than for ballistic systems. We provide a complete explanation of the nonlocal effect using a simple theoretical model based on the LandauerBüttiker formalism.

\footnotetext{
${ }^{*}$ E. Strambini ${ }^{\dagger}$, K.S. Makarenko ${ }^{\dagger}$ et al, in preparation
} 
Nonlocality is a key feature discriminating quantum and classical physics. Besides the famous Bell nonlocality [1], allowing for correlations between distant particles stronger than classically possible, the importance of another fundamental quantum nonlocality has been pointed out recently $[2,3]$. This dynamical nonlocality follows from the nonlocality of the quantum equations of motion, clearly manifest itself in branching geometries, and is at the base of all quantum interference phenomena, such as, for example, Young's double slit experiment and the Aharonov-Bohm (AB) effect [4]. Loss of dynamical nonlocality implies quenching of interference. It is well-known that this can occur due to (partial) collapse of the wave function due to a measurement, such as which-path detection [5]. However, other mechanisms affecting dynamical nonlocality have hardly been considered, although of crucial importance in many schemes for quantum information processing. Here, we present a fundamentally different pathway for losing dynamical localization in a solid-state quantum interference experiment where we demonstrate the crucial connection between dynamical nonlocality and the device geometry. We distinguish between the roles of quantum coherence and dynamical nonlocality in a diffusive $\mathrm{AB}$ interference experiment, using both local and nonlocal measurement geometries. The nonlocal interference is not only affected by decoherence, but also by a loss of dynamical nonlocality that, in contrast to which-path detection schemes, is based on a local reduction of the number of quantum conduction channels of the interferometer. With our measurements and theoretical model we demonstrate the fundamental nature of this mechanism, which is not limited by the diffusive transport regime, nor by the specific quantum wave-particle used (in our case electrons). Understanding the geometrical constraints protecting this kind of nonlocality is crucial when designing quantum networks for quantum information processing.

The $A B$ effect [4] is one of the most intriguing and insightful phenomena in quantum mechanics. If a quantum mechanical wave function, e.g. describing a propagating electron, is spatially split to enclose a region of magnetic flux, a phase difference develops, leading to quantum interference oscillations periodic in the magnetic flux quantum, $h / e$ ( $h$ is the Plank's constant and $e$ is the elementary charge). The $\mathrm{AB}$ effect has been experimentally demonstrated in a large number of systems, ranging from electron microscopy experiments $[6,7]$ to electron transport in mesoscopic rings [8-12]. As for the latter, the $A B$ effect has been shown both in the diffusive $[8,9]$ and ballistic $[10-12]$ regime with an oscillation amplitude of the order $e^{2} / h$, corresponding to the contribution of a single quantum conduction channel. It is remarkable that the $A B$ effect survives in metallic rings where, despite the many conduction channels and (elastic) scattering events, its amplitude remains of the same order. The $A B$ effect not only nicely 
demonstrates single electron interference, it also allows one to determine the electron coherence length $\left(l_{\varphi}\right)$ [13]. In this chapter, we present a set of electron-interference experiments in which besides the effect of decoherence we also observe the effect of loss of dynamical nonlocality [2, 3]. The dynamical nonlocality of electrons traversing the $\mathrm{AB}$ ring, induced by the branching of the conduction path, is, together with quantum coherence, the basic ingredient of the $\mathrm{AB}$ effect and, in fact, of any interference phenomenon.

Loss of dynamical nonlocality has been first demonstrated and tuned in which-path experiments $[5,14]$, one of the most remarkable manifestations of the complementarity principle. In these experiments, detection of the photon, respectively electron, traversing the two branches of the interferometer induces a collapse of the delocalized quantum state (wavelike) in a localized one (particle-like), resulting in quenching of the interference.

Here we investigate a new phenomenon affecting the dynamical nonlocality that, different from the which-path scheme, is inherent to the device geometry and originates from a reduction of the number of conduction channels instead of from the interaction with the external environment described above. This loss of dynamical nonlocality is not observed in a regular, local $\mathrm{AB}$ measurement geometry, but does play a significant role in the nonlocal geometry. In the nonlocal geometry, also the quantum nature of the side arms of the ring is crucial, as opposed to the local geometry, where only the ring itself matters. In the side arms, conduction channels can directly interact and merge, which is prevented in the ring. The implication of this fundamental difference will be discussed in detail below.

\subsection{Dynamic Nonlocality in a Diffusive Aharonov-Bohm Interferometer}

Figure 6.1a shows an image of a representative $\mathrm{Au} A B$ interferometer. More than ten devices of different size exhibited similar behaviour (Section 6.4). The $\mathrm{AB}$ ring is connected to two $100 \mathrm{~nm}$ long side arms, which each splits into two leads. This device layout allows for measuring the differential resistance $\left(\mathrm{d} V_{\mathrm{L}} / \mathrm{d} I=R_{\mathrm{L}}=R_{14,23}\right.$, current: $1 \rightarrow 4$, voltage: $\left.2 \leftrightarrow 3\right)$ in the conventional, local configuration (red, upper diagram in Fig. 6.1a). The local signal represented in the upper panel of Fig. 6.1b shows clear AB oscillations, see Fig. 6.1c, superimposed on a reproducible background of universal conductance fluctuations (UCF) (Section 1.2) [15]. The corresponding Fourier spectrum (Fig. 6.1d) clearly reveals the $\mathrm{AB}$ period $h / e$, as well as higher harmonics up to $h / 4 e$. In addition to $R_{\mathrm{L}}$, we have also measured the nonlocal differential resistance $\mathrm{d} V_{\mathrm{NL}} / \mathrm{d} I=R_{\mathrm{NL}}=R_{12,34}$, according to the scheme in the lower, blue 
diagram of Fig. 6.1a. Classically, one expects to measure a zero signal in this configuration (or exponentially small according to the Van der Pauw theorem [16]). However, in the quantum coherent transport regime, the signal may be nonzero $[15,17]$ as the electron wave function, dynamically delocalized in the system, can connect the current and voltage probes within a distance of a few coherence lengths. Indeed a nonlocal $A B$ effect has recently been measured in ballistic rings [18-22].

a
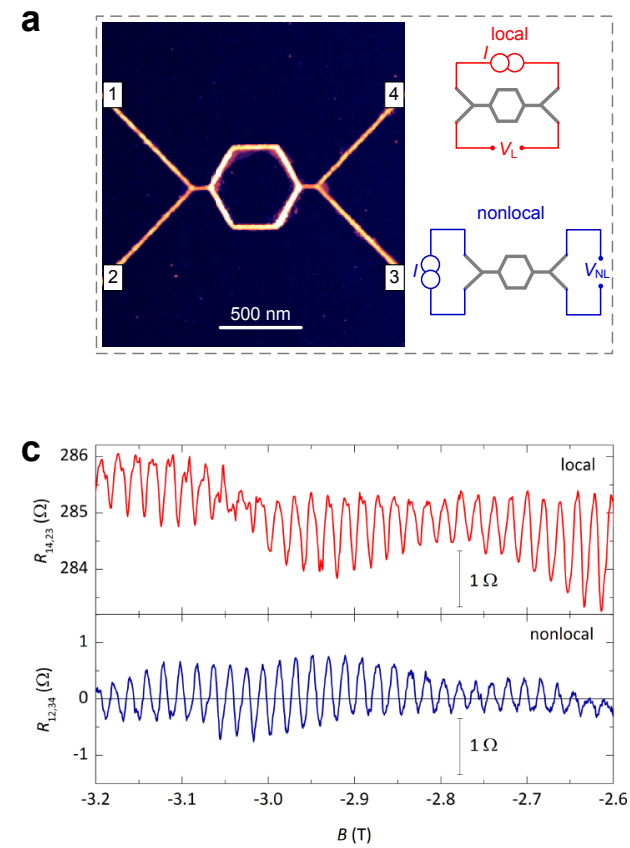

b
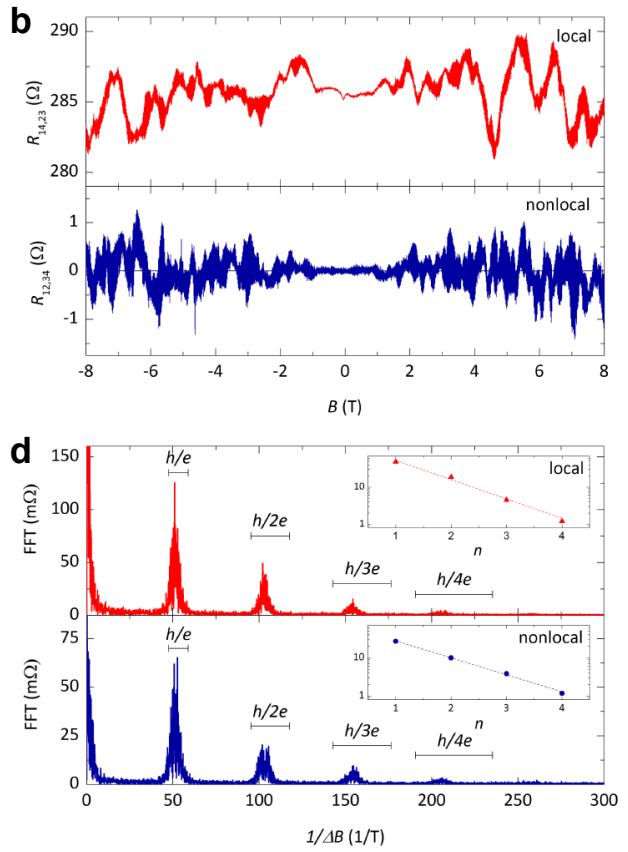

Figure 6.1. a, AFM image of the $A u A B$ interferometer and schematics of two measurement configurations: local 4-terminal (current: $1 \rightarrow 4$, voltage: $2 \leftrightarrow 3$ ) and nonlocal 4-terminal (current $1 \rightarrow 2$; voltage $3 \leftrightarrow 4$ ). b, $M R$ of the ring in a measured in local (red) and nonlocal (blue) geometries. c, Zoom-in of the magneto-resistance represented in $\mathbf{b}$. $\mathbf{d}$, Fourier transform of the data in a. The bounds for the flux periods $h / n e$ ( $n=1,2,3,4$ ) are calculated based on the inside and outside circumference of the device. ( $40 \mathrm{mK}, 30 \mathrm{nA}$ ac excitation current).

To the best of our knowledge, nonlocal $A B$ oscillations have never been observed in diffusive, metallic rings. We do, however, observe very clear 
nonlocal $\mathrm{AB}$ oscillations (Fig. 6.1b, blue) superimposed on reproducible fluctuations (UCF) around a zero average (Fig. 6.1c, blue). The Fourier spectrum shows the same four harmonics as for the local signal, but with roughly half the amplitude (Fig. 6.1d, lower panel).

For both the local and nonlocal configurations the amplitude of these Fourier peaks follows a clear exponential decay in the harmonic index $n$ (as shown in the insets of Fig. 6.1d), as the path length scales with $n$, corresponding to the number of times that the electron encloses the ring. This decay thus provides a direct measure of the electron coherence length $l_{\varphi}$ [13]. Using the power law $e^{-0.6 n C / l_{\varphi}}$, expected for a diffusive interferometer [17], where $\mathrm{C}$ is the ring circumference, giving rise to $l_{\varphi}=\sim 1.5 \mu \mathrm{m}$ for both the local and nonlocal configurations. The value of $l_{\varphi}$ is consistent with values found from weak localization and UCF measurements in mesoscopic Au structures at millikelvin temperatures [23]. Other similar rings show the same trend with $l_{\varphi}=1-2 \mu \mathrm{m}$ (Section 6.4), independent of sample size and temperature below 1 $\mathrm{K}$, as expected for gold [24]. Our results demonstrate that both the local and nonlocal interference are equally suppressed by decoherence inside the ring. Decoherence inside the side arms is only relevant for the nonlocal signal, leading to an additional damping $\mathrm{e}^{-1.1 d / l_{\varphi}}$ [17], where $d$ is the total length of the side arms. As a consequence, the amplitude of the nonlocal $\mathrm{AB}$ oscillations is smaller than that of the local $A B$ oscillations, as seen in Fig. 6.1d. This is in agreement with other studies on nonlocal interference $[15,18,25]$.

\subsection{Local and Nonlocal Measurements in the Multi-Terminal Quantum Interferometer}

To study the damping effect of the side arms on the nonlocal $A B$ oscillations in more detail, we measured $R_{\mathrm{NL}}$ and $R_{\mathrm{L}}$ in the multi-terminal interferometer shown in Fig. 6.2a, in which the side arm length $d$ can be varied (Fig. 6.2c,d). Clear AB oscillations are visible for $d=100 \mathrm{~nm}$ in both $R_{\mathrm{L}}\left(R_{09,14}\right.$ red plot in Fig. 6.2e) and $R_{\mathrm{NL}}\left(R_{12,34}\right.$ blue plot in Fig. 6.2f). For $R_{\mathrm{L}}$ the amplitude of the $\mathrm{AB}$ oscillations does not significantly change when the arm length $d$ is increased, see Fig. 6.2b. By contrast, the amplitude of the nonlocal $\mathrm{AB}$ oscillations is strongly suppressed with increasing $d$. The damping of the nonlocal oscillations can be described by the power low $e^{d / \xi}$, see Fig. 6.2b, where $\xi \sim 350 \pm 50 \mathrm{~nm}$ is the characteristic length scale of the damping. Interestingly, this damping is about three times faster than the theoretical expectation for a nonlocal measurement [17], which would give a value of $\sim 1.5 \mu \mathrm{m}$. We ascribe this large discrepancy to a loss of dynamical delocalization in the nonlocal geometry. 


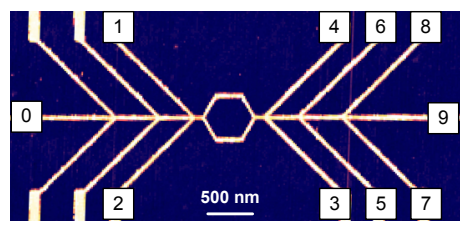

C
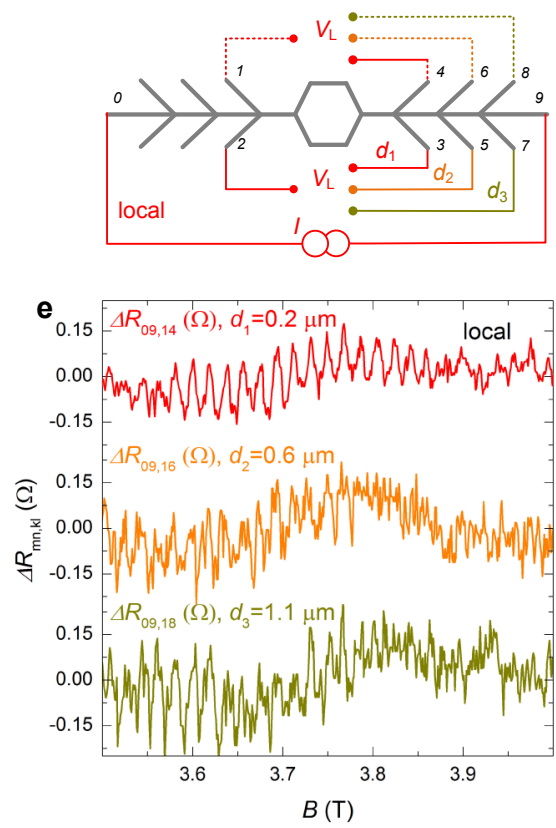

b

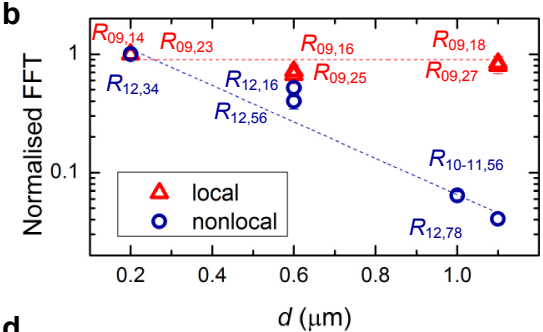

d
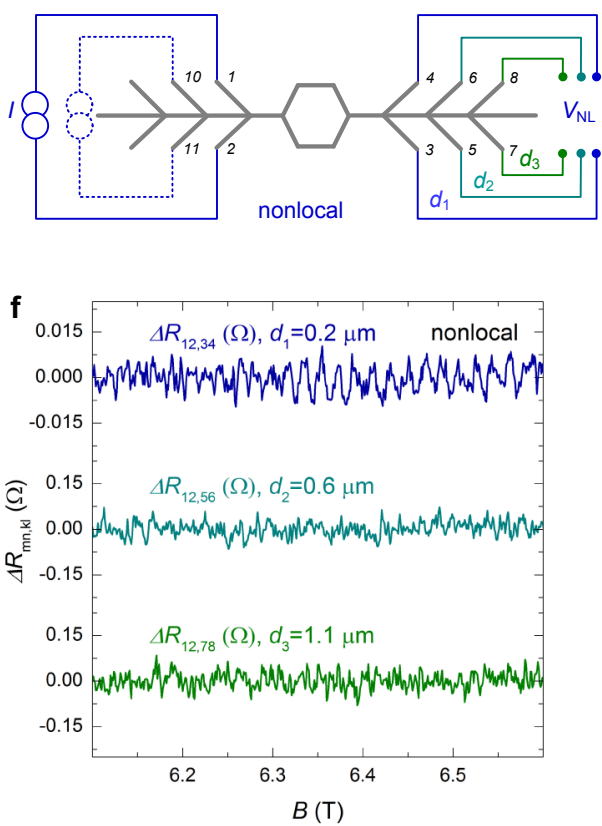

Figure 6.2. $\mathbf{a}, A F M$ image of the multi-terminal $A u A B$ interferometer. $\mathbf{b}$, Dependence of the quantum resistance oscillations on the distance $d$ between the probes and the ring showing the damping of the nonlocal voltages with increasing $d$. The amplitude of the quantum resistance oscillations and the error bars are determined from the Gaussian fit of the first peak in the Fourier transform of the data in e and f. c-d, Schematics of the local and nonlocal measurement geometries. e-f, Quantum resistance oscillations measured in local and nonlocal geometries.

Inside the $\mathrm{AB}$ ring itself merging of conduction channels in different branches of the ring is impossible, as they are simply spatially separated and therefore perfectly dynamically delocalized. In the local AB effect, loss of 
dynamical delocalization associated with merging of conduction channels inside the arms leading to the ring does not have any effect on the $A B$ amplitude, because only the ring itself is relevant for the interference. However, in the nonlocal $\mathrm{AB}$ effect, reduction of the amount of conduction channels inside the side arms has profound consequences for the interference, as follows straightforwardly from the model introduced below.

\subsection{Analytical Model Explaining the Nonlocal Effect*}

We use the Landauer-Büttiker (LB) formalism [26] to catch the essential physics that describes this quenching of interference in the nonlocal experiments first in the few-channel regime, and then extend the argument to our multichannel diffusive experimental system.

In the LB formalism the transport properties of a 4-terminal device are completely described by a unitary $4 \times 4$ scattering matrix $t_{\mathrm{ij}}$ representing the amplitude probabilities for an electron injected in the terminal $i$ to be transmitted to terminal $j$.

According to the scheme in the inset of Fig. 6.3a, the scattering matrix of a 4-terminal $\mathrm{AB}$ interferometer (without side arms) can be obtained by combining the scattering matrices of two $4 \times 4$ side nodes with a $4 \times 4$ phaseshifter representing the two branches of the ring, each of them shifting the electron phase by $\pm \phi / 2$, where $\phi$ is the $\mathrm{AB}$ phase. The 4-terminal resistances $R_{\mathrm{nm}, \mathrm{kl}}$ (current: $n \rightarrow m$, voltage: $k \rightarrow l$ ) are then extracted from the coefficients of the probability matrix $T_{\mathrm{ij}}=\left|t_{\mathrm{ij}}\right|^{2}$ according to the LB analytical formula [26]:

$$
R_{\mathrm{nm}, \mathrm{kl}}=\frac{h}{2 e^{2}} \cdot \frac{\left(T_{k m} T_{l n}-T_{k m} T_{l n}\right)}{D}
$$

where $D$ is a quantity including all of the $T_{\mathrm{ij}}$ 's coefficients.

Assuming a symmetric splitting probability in the two branches of the ring (Fig. 6.3a) and an exponential damping of the amplitude $\left(e^{-C / l_{\varphi}}\right)$ in both branches of the ring, to simulate decoherence, we obtain the $R_{\mathrm{L}}$ vs. $\phi$ and $R_{\mathrm{NL}}$ vs. $\phi$ plots shown in Fig. $6.3 \mathrm{~b}$. This single-channel model describes the fundamental characteristics of the experimentally observed $R_{\mathrm{L}}$ and $R_{\mathrm{NL}}$ well: a positive $R_{\mathrm{L}}$ exhibiting $\mathrm{AB}$ oscillations, and an $R_{\mathrm{NL}}$ oscillating between positive and negative values with a similar amplitude $\Delta R_{\mathrm{L}} \sim \Delta R_{\mathrm{NL}}$. This picture changes drastically when modelling the same ring with additional side arm, as represented in Fig. 6.3c. The addition of the single-channel side arm results in the complete quenching of all $R_{\mathrm{NL}}$, while the behaviour of $R_{\mathrm{L}}$ is not strongly affected, see Fig. $6.3 \mathrm{~d}$.

${ }^{*}$ The model was developed by Elia Strambini 
a

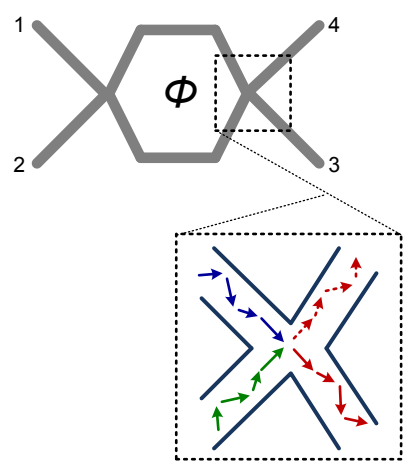

c

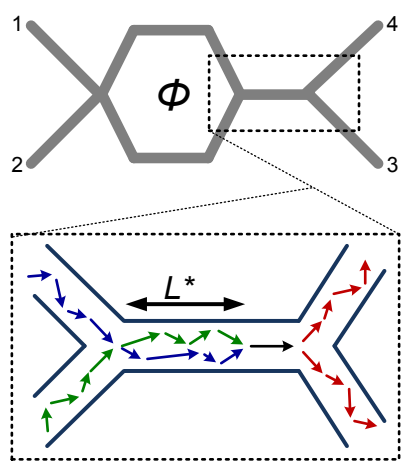

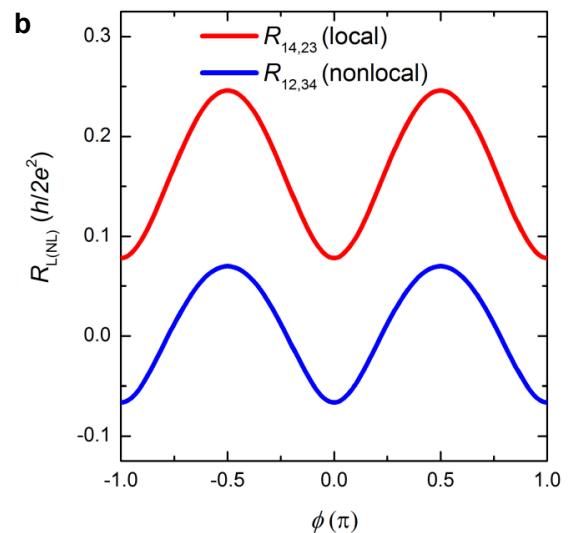

d

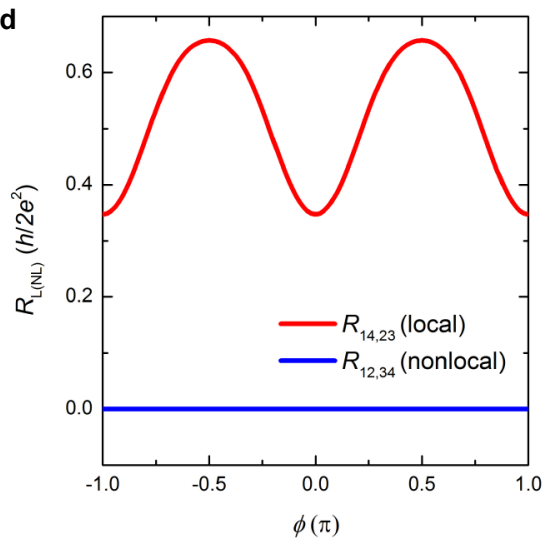

Figure 6.3. Consequence of the reduction of the amount of quantum channels in the side arm. a, Scheme of the simple 4-terminal $A B$ interferometer with a detailed representation of the second node of the interferometer in which the two electronic paths of the ring (green and blue arrows) interfere generating the two phase-correlated output channels represented by the red arrows (solid and dashed). b, Comparison between the 4-terminal local (red) and nonlocal (blue) resistances simulated as a function of the magnetic $A B$ phase $\phi$ for an $A B$ ring without side arms. $c$, Scheme of the 4-terminal $A B$ interferometer with one side arm allowing a reduction of two conduction channels into one after the characteristic distance $L^{*}$ and leading to quenching of the phase correlation between the two electronic paths of the ring, resulting in two phase-uncorrelated output channels represented by the solid red arrows. d, Comparison between the 4-terminal local (red) and nonlocal (blue) resistances simulated as a function of the magnetic $A B$ phase $\phi$ for the $A B$ ring with side arms. 
This detrimental effect on the nonlocal signal when forcing the conduction channels of both branches of the ring into the single channel of a side arm provides a strong clue for explaining the experimentally observed suppression of the $\mathrm{AB}$ oscillations in the nonlocal geometry. The forced reduction in the amount of conduction channels when the branches of the ring come together in the side arm generates an intrinsic constraint among the transmission coefficients representing the electrons injected in the side arm (e.g., $t_{13}, t_{14}, t_{23}, t_{24}$ in the schematic of Fig. 6.3c). The simple relation $t_{13} t_{24}=$ $t_{23} t_{14}$ holds, independent of the complexity or asymmetry of the system. From the definition of the 4-terminal resistance (Eq. 6.1), it is straightforward to note that this geometrical constraint is the origin of the quenching of all the nonlocal resistances (e.g., $R_{\mathrm{NL}}=R_{43,12} \propto T_{13} T_{24}-T_{14} T_{23}=0$ ), while it is only weakly affecting the local one. This geometrical effect and the corresponding quenching of $R_{\mathrm{NL}}$ reduces if the side arms are composed of more than a single conduction channel, as is expected in the diffusive case. This model is in agreement with experimental observations of the nonlocal $A B$ effect in ballistic systems [18-22], in which the nonlocal $A B$ oscillations have been reported for several transport channels. In our metallic devices, the side arms are composed of a multitude of interacting channels due to the diffusive electron motion, and the probability that scattering merges two otherwise distinct conduction channels into a single one (event represented in the scheme of Fig. 6.3c) is very high. The measured signal comes from the average of all the possible diffusive electron paths, in this average the paths that experience such a reduction do not contribute to interference, as demonstrated in our model. Due to the random distribution of electron paths in a diffusive metal, the probability for this event to occur is expected to scale exponentially with the length of the side arm $\left(\propto 1-e^{-d / L^{*}}\right.$, where $L^{*}$ is the characteristic length of this event) and as a consequence the nonlocal signal is damped within the same length scale $\left(e^{-d / L^{*}}\right)$ and additionally to the damping induced by decoherence [17]. This behaviour is in agreement with the strong exponential damping of the $R_{\mathrm{NL}}$ shown in Fig. 6.2c from which we can estimate $L^{*}=\left(1 / \xi-1.1 / l_{\varphi}\right)^{-1} \approx 450 \pm 50 \mathrm{~nm}$.

\subsection{Characterisation of Aharonov-Bohm Interferometers}

\subsubsection{Evaluation of the Coherence Length}

Figure 6.4 shows the fast Fourier transform (FFT) of the magneto-resistance oscillations measured through the device in Fig. 6.1a in the local and the nonlocal configurations, after subtracting a polynomial fit (to remove the 
aperiodic background fluctuations, UCF). Six clear peaks corresponding to the $h /$ ne harmonics can be observed.

The amplitude of the $\mathrm{AB}$ oscillations decays exponentially with the coherence length of the system according to these relations [17]:

$$
\begin{array}{ll}
\text { for } R_{\mathrm{L}}: & A \propto e^{-0.6 \frac{n C}{l_{\varphi}}}, \\
\text { for } R_{\mathrm{NL}}: & A \propto e^{-\left(0.6 \frac{n C}{l_{\varphi}}+1.1 \frac{d}{l_{\varphi}}\right)},
\end{array}
$$

where $C$ is the circumference of $\mathrm{AB}$ interferometer, $d$ is the total length of the side arms, $l_{\varphi}$ is coherence length. Based on Eqs. (6.2)-(6.3) we can extract $l_{\varphi}$ by fitting the peak amplitude in the Fourier transform (see insets of Fig. 6.4a,b). We obtained a coherence length $l_{\varphi}$ of $\sim 1.5 \mu \mathrm{m}$ for both local and nonlocal configurations.
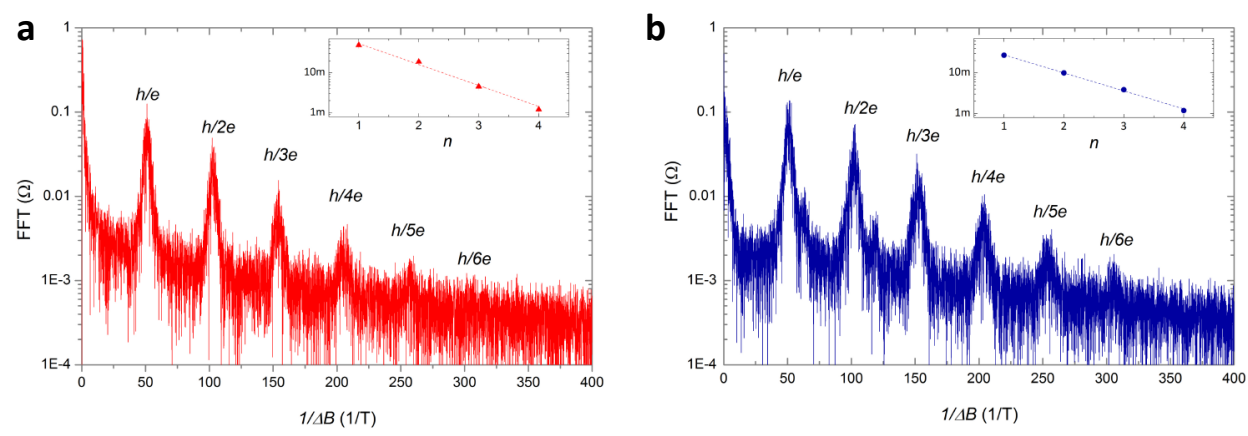

Figure 6.4. Characterisation of $A u A B$ interferometer. a, Representative FFT of $R_{\mathrm{NL}}$ of the interferometer in Fig. 6.1a, showing up to six harmonics. b, FFT peak amplitudes of the magneto-resistance, represented in Fig. 1 b, vs. harmonic index $n$. Inset in $\mathbf{b}$ shows FFT peak amplitude of the $R_{\mathrm{NL}}$ vs. $n$ characteristic of the $\mathrm{Au} \mathrm{AB}$ interferometer shown in Figs.2d ( $40 \mathrm{mK}, 30 \mathrm{nA}$ ac excitation current).

\subsubsection{Temperature Dependence of the AB Oscillation Amplitude}

Temperature dependence of the $\mathrm{AB}$ oscillation amplitude (Figs. 6.5) shows that although the amplitude of the FFT peaks decreases, the ratio between the different harmonics remains almost the same. From this ratio the coherence length can be extracted. We observed that despite the constant coherence length below $1 \mathrm{~K}$ (Fig. 6.6) the amplitude of the oscillations is damping what can be addressed to a phase averaging due to the thermal broadening. 

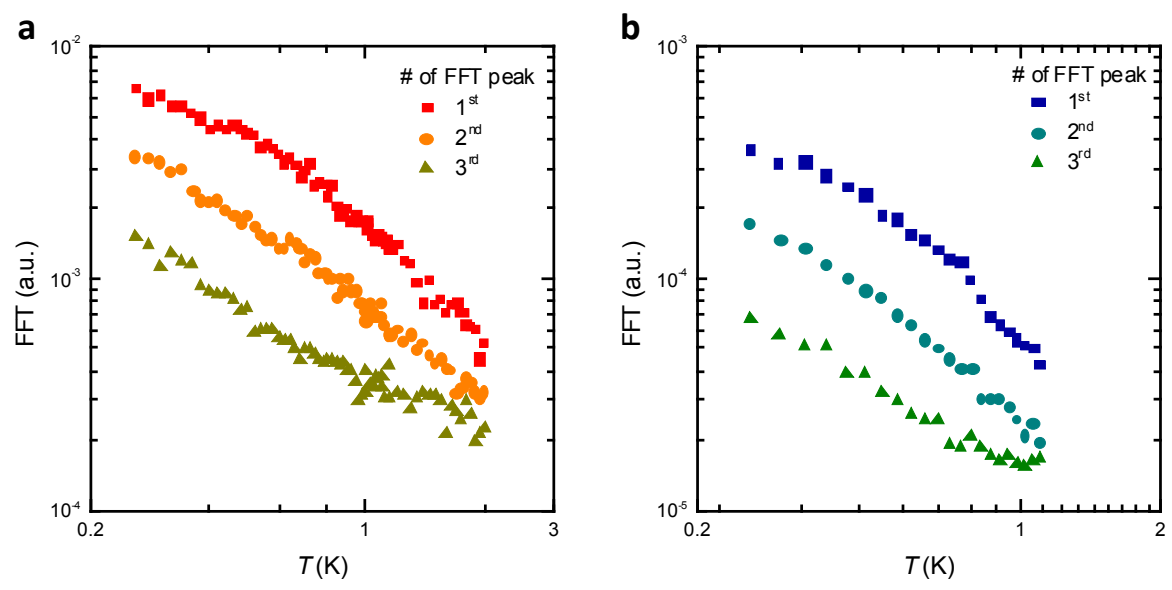

Figure 6.5. Temperature dependence of the $A B$ oscillation amplitude: dependence of the $h / e$ oscillations for the local (a) and the nonlocal (b) geometries (30 nA ac excitation current).
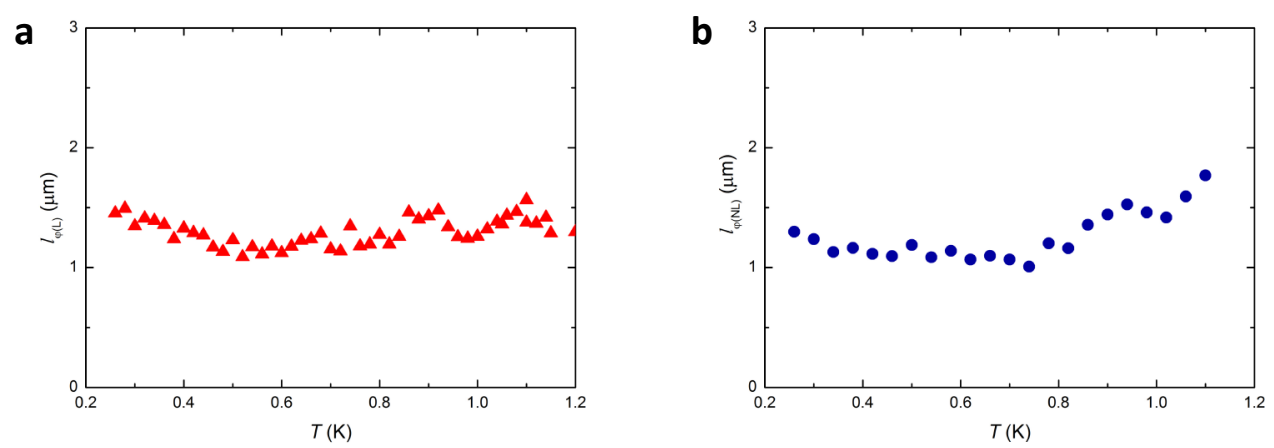

Figure 6.6. Temperature dependence of the coherence length of the $A B$ interferometer in Fig. 6.1a: for the local (a) and the nonlocal (b) configurations (30 nA ac excitation current).

\subsubsection{Excitation Current Dependence of the AB Oscillation Amplitude}

Figure 6.7 shows an excitation current $\left(I_{\mathrm{ac}}\right)$ dependence of the $\mathrm{AB}$ oscillation amplitudes for the $1^{\text {st }}, 2^{\text {nd }}$ and $3^{\text {rd }}$ FFT peaks, measured in the local configuration. The amplitude of the FFT peaks does not change for excitation 
currents below $\sim 50 \mathrm{nA}$ (see the crossing point of two red dashed guiding lines in Fig. 6.7). From this $I_{\mathrm{ac}}$ value and the $\mathrm{AB}$ ring's resistance $(\sim 282 \Omega)$ the corresponding energy broadening is $\Delta \mathrm{E} \sim 14 \mu \mathrm{eV}$ from which it is possible to estimate an effective electron temperature $T_{\mathrm{e}} \simeq 50 \mathrm{mK}\left(\Delta \mathrm{E}=3.5 \mathrm{k} T_{\mathrm{e}}\right)$. Above this point the peak amplitudes decay due to phase averaging or heating induced by the excitation current.

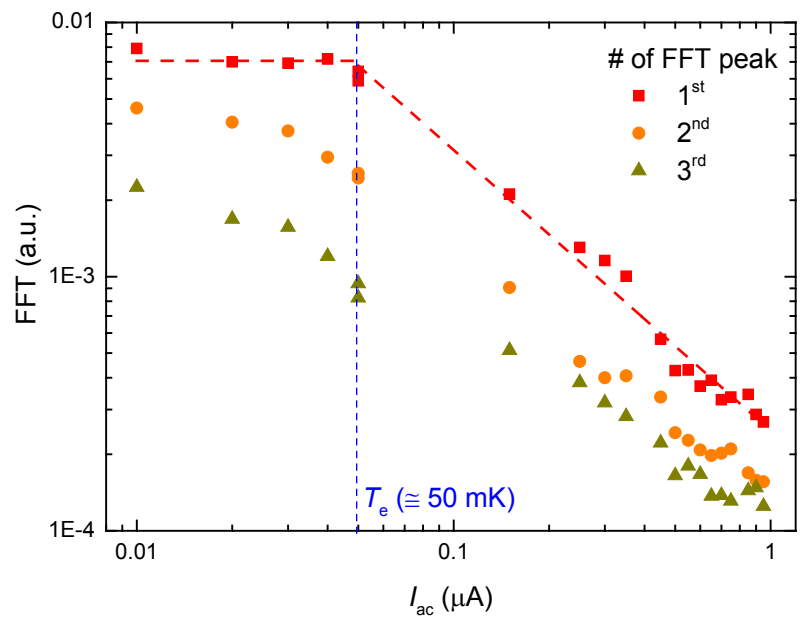

Figure 6.7. Excitation current dependence represents damping of the $1^{\text {st }}$, the $2^{\text {nd }}$ and the $3^{\text {rd }}$ FFT peaks of the $A B$ oscillations measured for the $A B$ ring in Fig. 6.1a in the local configuration ( $T=40 \mathrm{mK}$ ).

\subsubsection{Size Dependence of the AB Oscillations}

The $\mathrm{AB}$ period is determined by the ring's enclosed area $S_{\text {in }}, \Delta B=h / e S_{\text {in }}$ [4]. Figure 6.8 shows electrical measurements on two $A B$ interferometers of a different size (490 \pm 10 (Figs 6.8a,b in black) and $590 \pm 10 \mathrm{~nm}$ (Figs 6.8a in red, Fig. 6.8b, in blue)) in diameter) for local (Fig. 6.8a) and nonlocal (Fig. 6.8b) configurations. Calculated from the geometry of the $\mathrm{AB}$ rings oscillation frequencies $1 / \Delta B=e / h S_{\text {in }}$ are $(52 \pm 2)$ and $(75 \pm 2) \mathrm{T}^{-1}$ for the $(490 \pm 10)$ and $(590 \pm 10) \mathrm{nm}$ diameter interferometers, respectively, are in agreement with extracted from FFT peaks $\mathrm{AB}$ period of $\sim 51$ and $\sim 74 \mathrm{~T}^{-1}$ (for 490 and $590 \mathrm{~nm}$, respectively) (Figs. 6.8c,d). 
a

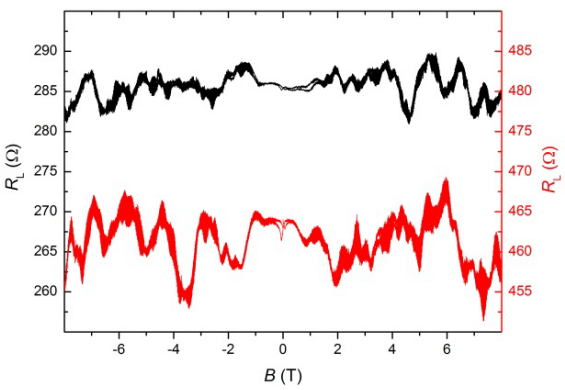

c

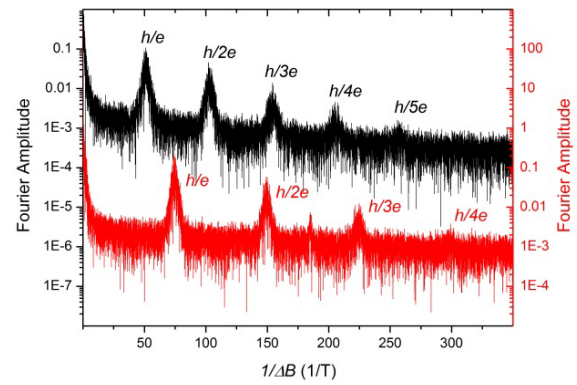

b

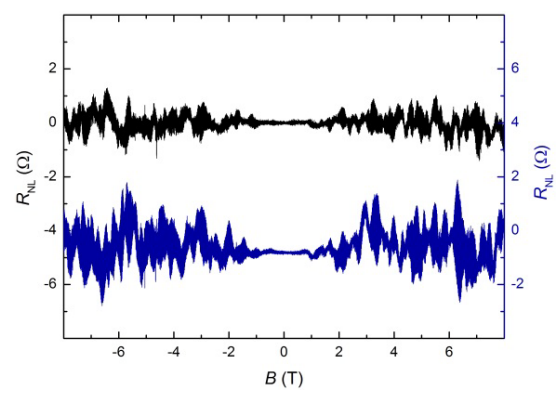

d

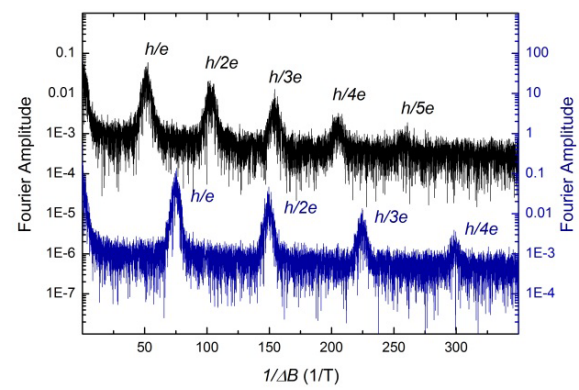

Figure 6.8. Comparison between $A B$ interferometers of two diameters for local and nonlocal geometries. $\mathbf{a}-\mathbf{b}$, Magneto-resistance of two $A u A B$ interferometers with a diameter of $\sim 490 \mathrm{~nm}$ (black) and $\sim 590 \mathrm{~nm}$ (red and blue) measured in the local (a) and the nonlocal (b) geometries. c-d, Fourier transform of the data presented in $\mathbf{a}$ and $\mathbf{b}$, respectively.

\section{Conclusions}

We have thus shown a new aspect of the dynamical nonlocality of electrons in a quantum circuit that is solely governed by geometric aspects and not by external measurement. Understanding this geometrical constraint is essential for the optimal design of any quantum circuit based on the dynamical nonlocality of the electrons. Moreover, as deduced from our model, the strong link between geometry and nonlocality is a universal property of quantum dynamics not limited by the diffusive transport regime of our experiment, nor by the specific quantum wave-particle used (in our case electrons). We believe that these results will trigger further investigation of the fundamental properties of quantum dynamics and of its application in quantum information processing. 


\section{REFERENCES}

[1] J. S. Bell, "On the Einstein Podolsky Rosen Paradox," Physics, vol. 1, pp. 195-200, 1964.

[2] S. Popescu, "Dynamical quantum non-locality," Nature Physics, vol. 6, pp. 151-153, 03//print 2010.

[3] S. Popescu, "Nonlocality beyond quantum mechanics," Nature Physics, vol. 10, pp. 264-270, 04//print 2014.

[4] Y. Aharonov and D. Bohm, "Significance of Electromagnetic Potentials in the Quantum Theory," Physical Review, vol. 115, pp. 485-491, 08/01/ 1959.

[5] E. Buks, R. Schuster, M. Heiblum, D. Mahalu, and V. Umansky, "Dephasing in electron interference by a /which-path/' detector," Nature, vol. 391, pp. 871-874, 02/26/print 1998.

[6] R. G. Chambers, "Shift of an Electron Interference Pattern by Enclosed Magnetic Flux," Physical Review Letters, vol. 5, pp. 3-5, 07/01/ 1960.

[7] A. Tonomura, T. Matsuda, R. Suzuki, A. Fukuhara, N. Osakabe, H. Umezaki, et al., "Observation of Aharonov-Bohm Effect by Electron Holography," Physical Review Letters, vol. 48, pp. 1443-1446, 05/24/ 1982.

[8] R. A. Webb, S. Washburn, C. P. Umbach, and R. B. Laibowitz, "Observation of h/e Aharonov-Bohm oscillations in normal-metal rings," Physical Review Letters, vol. 54, pp. 2696-2699, Jun 241985.

[9] A. van Oudenaarden, M. H. Devoret, Y. V. Nazarov, and J. E. Mooij, "Magneto-electric Aharonov-Bohm effect in metal rings," Nature, vol. 391, pp. 768-770, 02/19/print 1998.

[10] G. Timp, A. M. Chang, J. E. Cunningham, T. Y. Chang, P. Mankiewich, R. Behringer, et al., "Observation of the Aharonov-Bohm effect for \$ $\{$ \lomega\$ \{\}$\} \_\{\mathrm{c}\} \$ \$$ tau $\{\{\}$ gt; $1, "$ Physical Review Letters, vol. 58, pp. 2814-2817, 06/29/ 1987.

[11] W. G. van der Wiel, Y. V. Nazarov, S. De Franceschi, T. Fujisawa, J. M. Elzerman, E. W. G. M. Huizeling, et al., "Electromagnetic AharonovBohm effect in a two-dimensional electron gas ring," Physical Review B, vol. 67 , p. $033307,01 / 29 / 2003$.

[12] E. Strambini, V. Piazza, G. Biasiol, L. Sorba, and F. Beltram, "Impact of classical forces and decoherence in multiterminal Aharonov-Bohm networks," Physical Review B, vol. 79, p. 195443, 05/29/2009.

[13] A. E. Hansen, A. Kristensen, S. Pedersen, C. B. Sørensen, and P. E. Lindelof, "Mesoscopic decoherence in Aharonov-Bohm rings," Physical Review B, vol. 64, p. 045327, 07/05/ 2001.

[14] X. Y. Zou, L. J. Wang, and L. Mandel, "Induced coherence and indistinguishability in optical interference," Physical Review Letters, vol. 67, pp. 318-321, 07/15/ 1991. 
[15] A. Benoit, C. P. Umbach, R. B. Laibowitz, and R. A. Webb, "LengthIndependent Voltage Fluctuations in Small Devices," Physical Review Letters, vol. 58, pp. 2343-2346, 06/01/ 1987.

[16] L. J. van der Pauw, "A method of measuring specific resistivity and Hall effect of discs of arbitrary shape," Philips Research Reports, vol. 13, pp. 19, 1958.

[17] D. P. DiVincenzo and C. L. Kane, "Voltage fluctuations in mesoscopic metal rings and wires," Physical Review B, vol. 38, pp. 3006-3015, 08/15/ 1988.

[18] K. Kobayashi, H. Aikawa, S. Katsumoto, and Y. Iye, "ProbeConfiguration-Dependent Decoherence in an Aharonov-Bohm Ring," Journal of the Physical Society of Japan, vol. 71, pp. 2094-2097, 2002/09/15 2002.

[19] S. S. Buchholz, S. F. Fischer, U. Kunze, D. Reuter, and A. D. Wieck, "Nonlocal Aharonov-Bohm conductance oscillations in an asymmetric quantum ring," Applied Physics Letters, vol. 94, p. 022107, 2009.

[20] S. S. Buchholz, S. F. Fischer, U. Kunze, M. Bell, D. Reuter, and A. D. Wieck, "Control of the transmission phase in an asymmetric four-terminal Aharonov-Bohm interferometer," Physical Review B, vol. 82, p. 045432, 07/30/ 2010.

[21] K.-T. Lin, Y. Lin, C. C. Chi, J. C. Chen, T. Ueda, and S. Komiyama, "Temperature- and current-dependent dephasing in an Aharonov-Bohm ring," Physical Review B, vol. 81, p. 035312, 01/08/ 2010.

[22] K.-T. Lin, Y. Lin, C. C. Chi, and J. C. Chen, "Asymmetric transmissioninduced probe-configuration-dependent dephasing in an Aharonov-Bohm ring," Physical Review B, vol. 84, p. 235404, 12/01/ 2011.

[23] L. Saminadayar, P. Mohanty, R. A. Webb, P. Degiovanni, and C. Bäuerle, "Electron coherence at low temperatures: The role of magnetic impurities," Physica E: Low-dimensional Systems and Nanostructures, vol. 40, pp. 1224, 10// 2007.

[24] P. Mohanty, E. M. Q. Jariwala, and R. A. Webb, "Intrinsic Decoherence in Mesoscopic Systems," Physical Review Letters, vol. 78, pp. 3366-3369, 04/28/ 1997.

[25] H. Haucke, S. Washburn, A. D. Benoit, C. P. Umbach, and R. A. Webb, "Universal scaling of nonlocal and local resistance fluctuations in small wires," Physical Review B, vol. 41, pp. 12454-12461, 06/15/ 1990.

[26] M. Büttiker, "Four-Terminal Phase-Coherent Conductance," Physical Review Letters, vol. 57, pp. 1761-1764, 10/06/ 1986. 


\section{Chapter 7 COHERENT ElECTRON Transport THROUGH HYBRID AHARONOV-BOHM INTERFEROMETERS}

This chapter is dedicated to the experimental observation of potentially coherent electron transport through molecular layers. In an Aharonov-Bohm (AB) interferometer based on a pair of Au nanoparticles (NPS) coupled in parallel between two Au nanorods (NRs), we show an indication of the coherent electron transport through 1,8-octanedithiol self-assembled monolayers (SAMs). Based on the obtained result, we propose novel geometries for hybrid $A B$ interferometers containing embedded molecular barriers, which may allow for a consistent study of coherent electron transport through molecules. 


\subsection{Coherent Transport through Organic Molecular Layers}

Although coherent electron transport in inorganic systems is experimentally well studied by now [1-4], the coherence of electron transport through organic molecules is experimentally much less explored [5-9]. Understanding the transport through molecular systems has attracted considerable interest due to their potential application in nanoelectronics. It is a fair and fascinating question to ask to what extent transport through organic molecules is coherent and how the coherence depends on the molecule's characteristics (e.g. length, chemical composition, side groups, chemical bonding), as well as on external parameters (e.g. temperature, electrostatic potential, magnetic field). Our objective is to include monolayers of organic molecules in electron interferometer geometry, allowing for a systematic study of charge coherence in molecular systems.

\subsection{Aharonov-Bohm Oscillations in Gold Rings Bridged by Gold Nanoparticles}

The first step towards studying interference effects in hybrid inorganicorganic $\mathrm{AB}$ interferometer is to show coherent transport through a bottom-up $\mathrm{AB}$ interferometer without molecular junctions (so in fully inorganic structures). Using dielectrophoresis (DEP), described in chapter 2, we bridged $30 \mathrm{~nm}$ gaps in $\mathrm{Au} \mathrm{AB}$ interferometer with a diameter of $500 \mathrm{~nm}$ by a few nonfunctionalised ("naked") Au NPs, which are on average $20 \mathrm{~nm}$ in diameter (inset in Fig. 7.1).

Figure 7.1 shows clear AB oscillations in a magnetic field between 6 and $6.5 \mathrm{~T}$, measured in a four-wire configuration. The Fourier transform of these oscillations, after subtracting a polynomial fit (to remove the background fluctuations, UCF (Section 1.2)), is shown in Fig. 7.2, where three clear peaks at $50 \mathrm{~T}^{-1}, 102 \mathrm{~T}^{-1}$ and $150 \mathrm{~T}^{-1}$ can be observed, which correspond to the first $(h / e)$, the second $(h / 2 e)$ and the third $(h / 3 e)$ harmonics. From these equidistant FFT peaks the $A B$ period $\Delta B$ is estimated to be $20 \mathrm{mT}$, in good agreement with the calculated period of $(19 \pm 2) \mathrm{mT}$ for the geometry of the measured ring. The widths of the $h / e, h / 2 e$ and $h / 3 e$ are calculated from the ring's aspect ratio and are indicated by bars in Fig. 7.2.

This experimental observation of $\mathrm{AB}$ oscillations in $\mathrm{Au}$ rings realized by combining bottom-up (self-assembly of NPs) and top-down (electron beam lithography) approaches (inset in Fig. 7.1) suggests that the proposed novel hybrid $\mathrm{Au} A B$ ring (see below, Section 7.4) can be used to probe the coherent electron transport properties of the organic molecules of interest. 


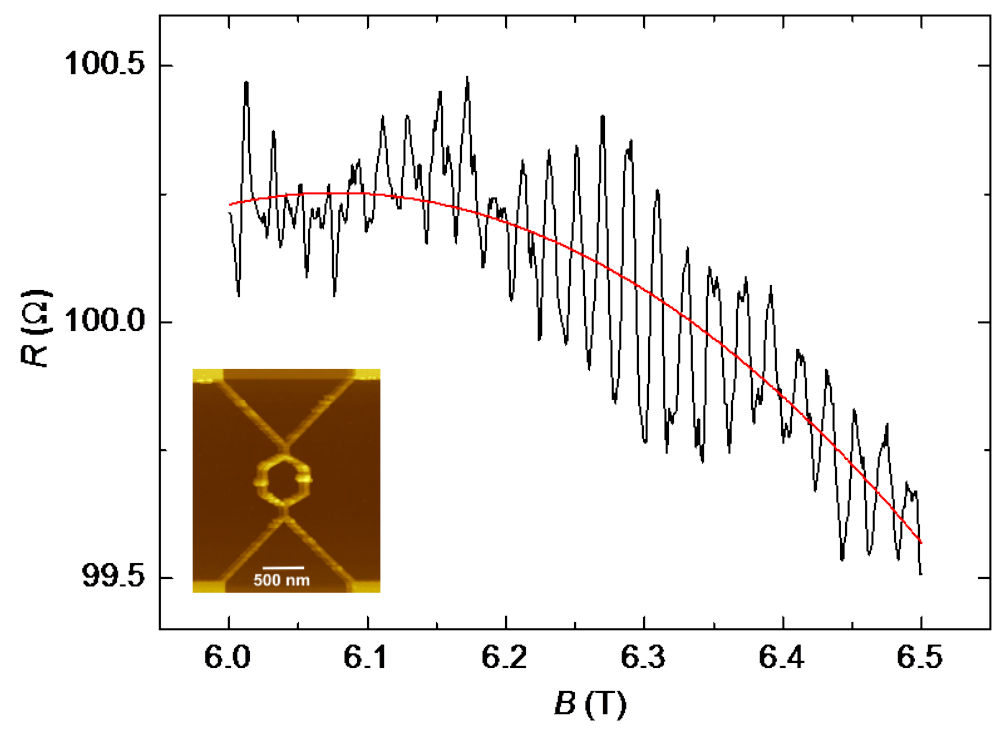

Figure 7.1. $A B$ oscillations observed in a $500 \mathrm{~nm}$ diameter $A u A B$ ring (AFM image in the inset), closed by few $20 \mathrm{~nm}$ diameter nonfunctionalised Au NPs, bridged via DEP (black line), and polynomial fit of the data (red line) ( $32 \mathrm{mK}, 50 \mathrm{nA}$ ac excitation current at $17.7 \mathrm{~Hz}$ ).

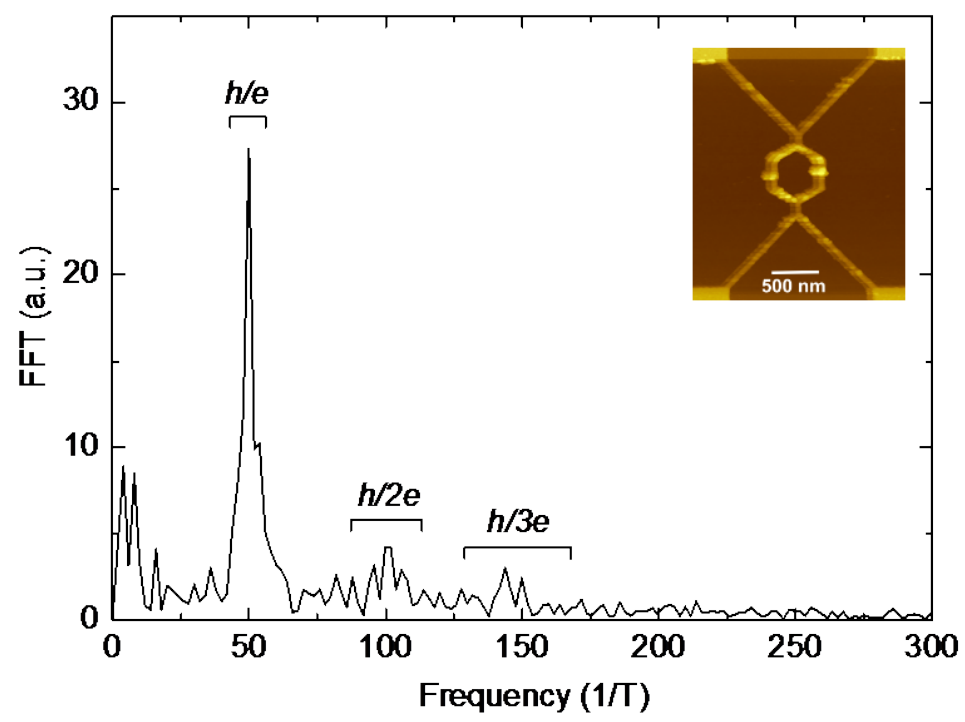

Figure 7.2. Fourier transform of the $A B$ oscillation in Fig. 7.1, after subtracting the polynomial fit. The bars to each peak indicate the frequency bandwidth for $h / e, h / 2 e$, and $h / 3 e$ oscillations. The inset shows AFM image of the measured ring. 


\subsection{Interference Effects in Aharonov-Bohm Ring Created via Selective Chemical Interaction}

Two Au NPs assembled in parallel between two Au nanorods (NRs) (Chapters 4-5) and decoupled from them by a molecular barrier (1,8-octanedithiol) form a branching geometry that could possibly act as a two-path interferometer. Figure 7.3 shows a scanning electron microscopy (SEM) image of the device.

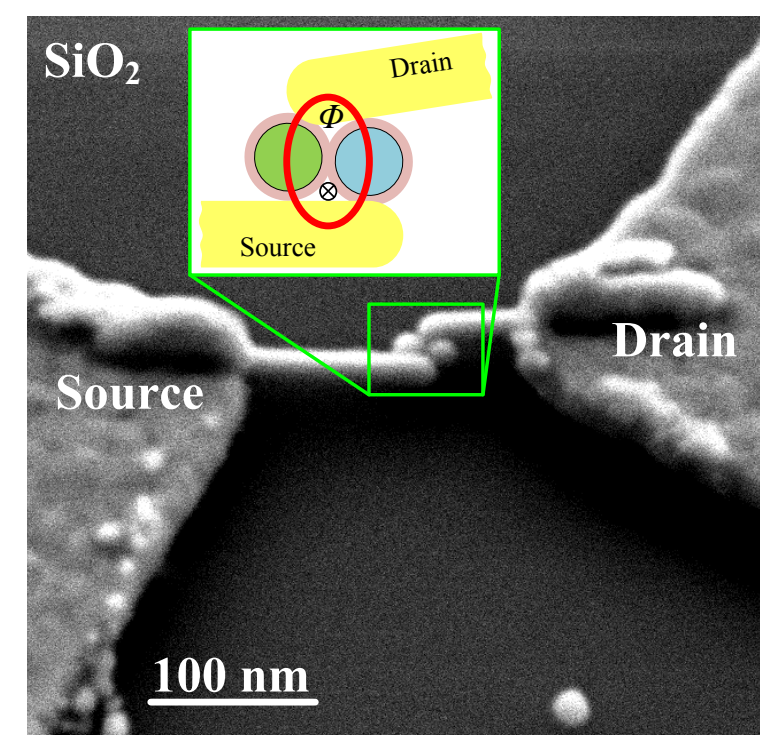

Figure 7.3. SEM image of the two $20 \mathrm{~nm}$ Au NPs enclosed between two Au NRs ( $25 \mathrm{~nm}$ in diameter and $250 \mathrm{~nm}$ long) (Chapters 4-5). The inset shows schematic of the device which may act as an $A B$ interferometer.

Figure 7.4 shows the differential conductance $(\mathrm{d} I / \mathrm{d} V)$ through the device in Fig. 7.3 as a function of the source-drain voltage $\left(V_{\text {bias }}\right)$ and back gate voltage $\left(V_{\text {gate }}\right)$. When an electron is able to move simultaneously through both branches of the $\mathrm{AB}$ interferometer, the $\mathrm{AB}$ effect may be observed. For our device (Fig. 7.3) this condition will be fulfilled when both dots are out of the Coulomb blockade region. In chapter 5 we have discussed that electron transport through one Coulomb island strongly depends on the transport through the other island coupled in parallel. Therefore, we carefully chose the position inside the stability diagram (Chapter 5, Figs. 5.3-5.6). Figure 7.5 shows conductance oscillations in a magnetic field piercing the device. These oscillations are reproducible in time and were tested for a different step sizes of the magnetic field sweep to exclude spurious effects. The inset in Fig.7.5 
shows a dummy time sweep where no oscillations are present. This suggests that the observed periodic magnetic field dependence (yellow dashed sine curve fit in Fig. 7.5) is caused by interference of electrons traversing the device.

The inset in Fig. 7.4 shows a schematic diagram of the electrochemical potentials $\mu_{1(2)}(N, M)$ (Section 1.3) in the islands and leads for the chosen optimal configuration, at which electrons can traverse both islands.

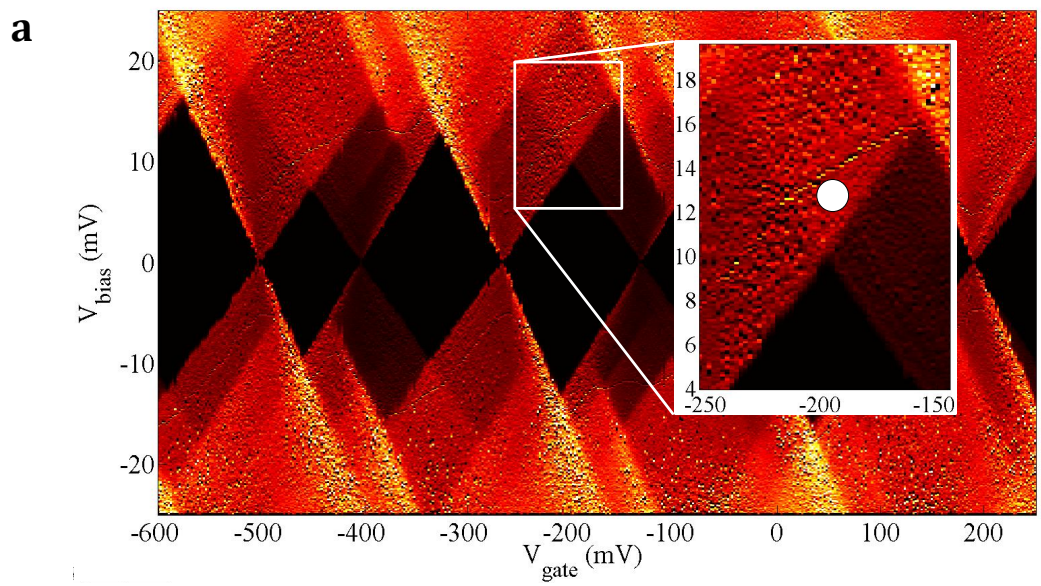

b

$N-1$

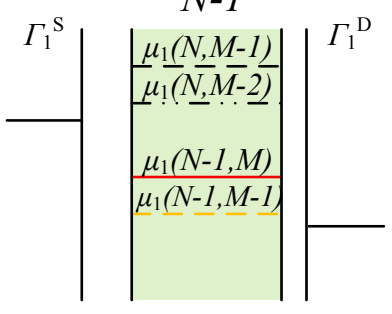

$M$

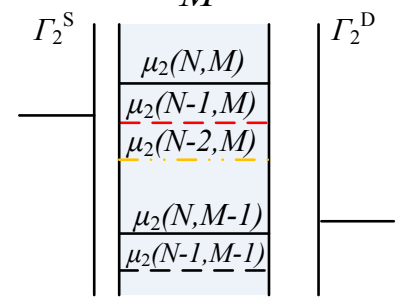

Figure 7.4. a, Differential conductance $(\mathrm{d} / \mathrm{d} V)$ as a function of the source-drain voltage $\left(V_{\text {bias }}\right)$ and back gate voltage $\left(V_{\text {gate }}\right)$, showing electron transport through a pair of Coulomb islands in parallel, depicted in Fig. 7.3. The inset in a shows a zoom-in of the chosen measurement region (high-lighted by the white circle). b, Stability diagram showing electrochemical potentials $\mu_{1(2)}(N, M)$ corresponding to two Coulomb islands (Section 1.3) at the chosen region in the inset in a (Section 5.2). ( $\mathrm{T}<15 \mathrm{mK})$

We also observe a change of the magnetic field oscillations for different gate and bias voltages (Figs 7.6 and 7.7, respectively). Such 
behaviour was observed in metal $\mathrm{AB}$ rings containing tunnel barriers in its branches and may be addressed to the electric modification of the electron phase [4].

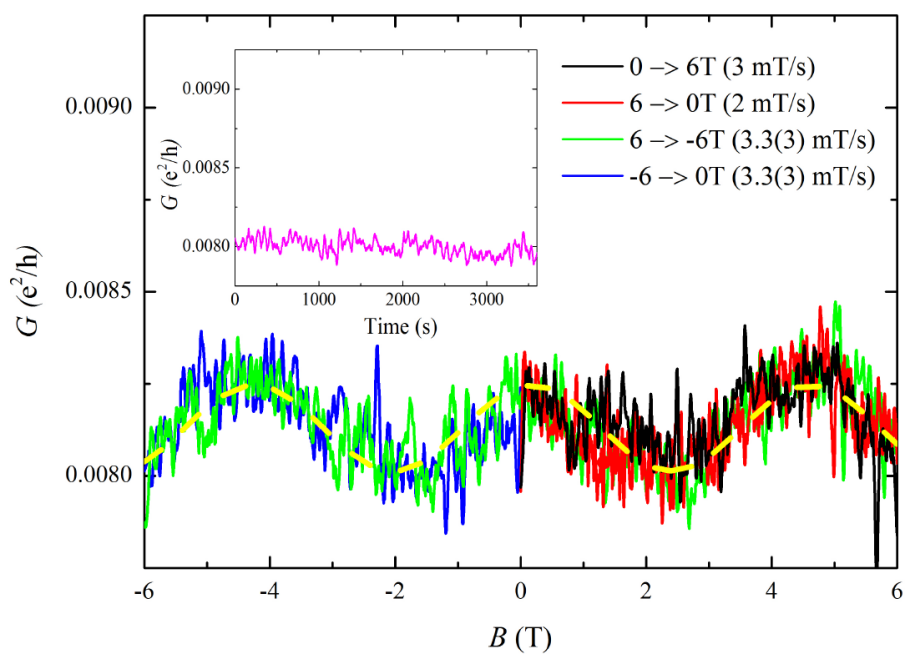

Figure 7.5. Conductance oscillations through the double Coulomb islands coupled in parallel, shown for different step sizes and directions of the magnetic field sweep. Yellow dashed line is sine curve fits. Inset: dummy time sweep showing no periodic oscillations. $\left(V_{\text {bias }}=13 \mathrm{mV}, V_{\text {gate }}=-190\right.$ $\mathrm{mV}, V_{\mathrm{AC}}=100 \mu \mathrm{V}$ at $\left.17.7 \mathrm{~Hz}, T=30 \mathrm{mK}\right)$.

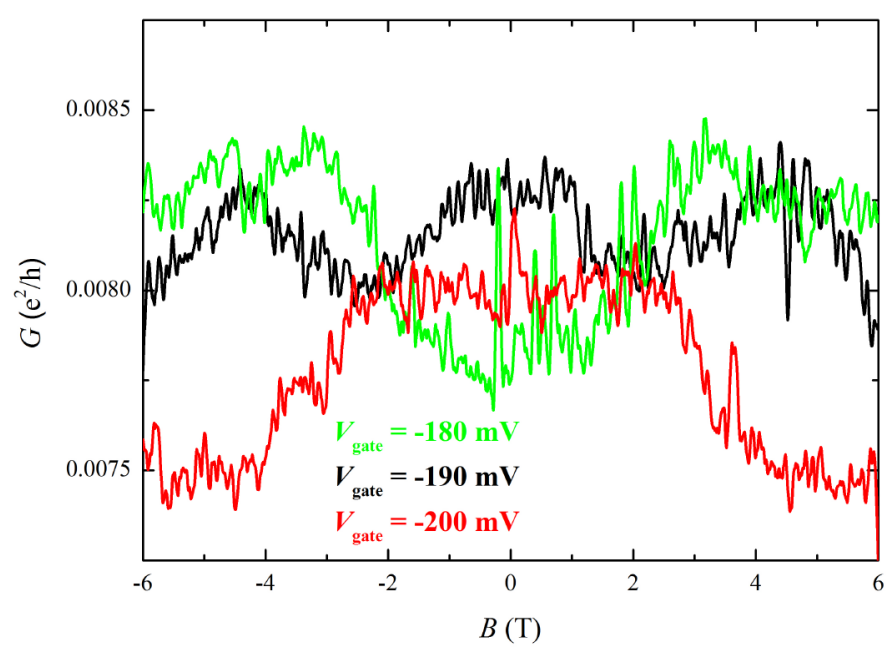

Figure 7.6. Conductance oscillations at different gate voltages $\left(V_{\text {gate }}\right)$ showing a change in the behaviour for the fixed bias and excitation voltages $\left(V_{\text {bias }}=13 \mathrm{mV}, V_{\mathrm{AC}}=100 \mu \mathrm{V}\right.$ at $17.7 \mathrm{~Hz}, T=30 \mathrm{mK}$ ). 


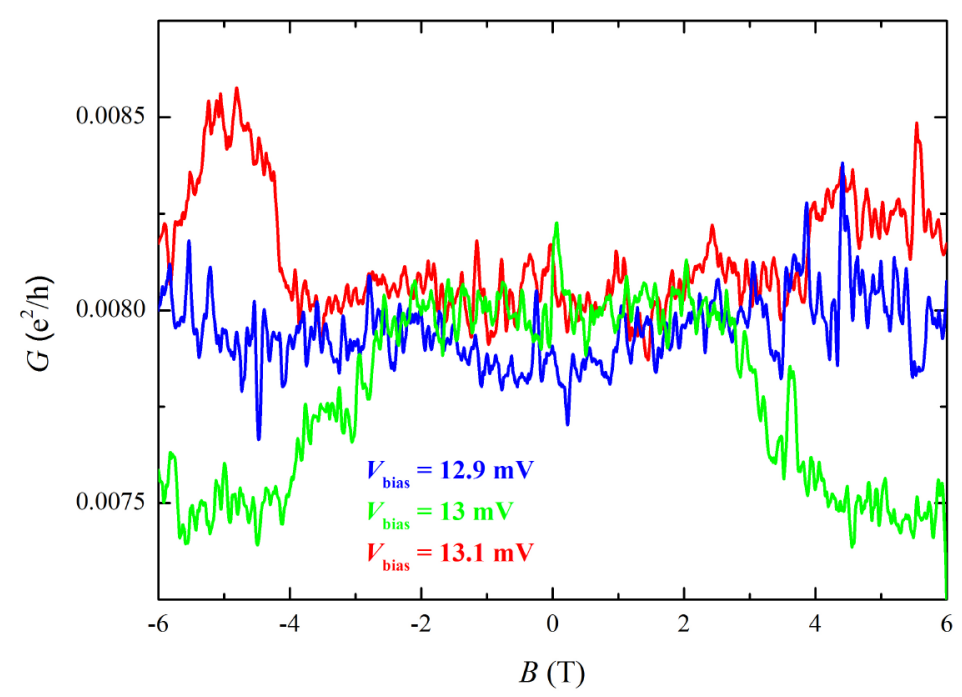

Figure 7.7. Conductance oscillations at different bias voltages $\left(V_{\text {bias }}\right)$ showing a change in the behaviour for the fixed gate and excitation voltages ( $V_{\text {gate }}=-190 \mathrm{mV}, V_{\mathrm{AC}}=100 \mu \mathrm{V}$ at $17.7 \mathrm{~Hz}, T=30 \mathrm{mK}$ ).

a

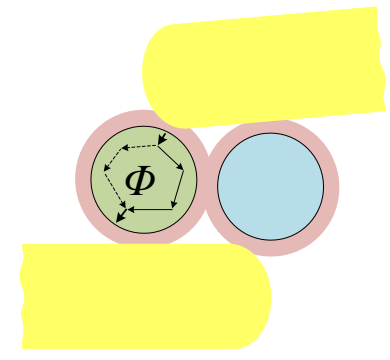

C

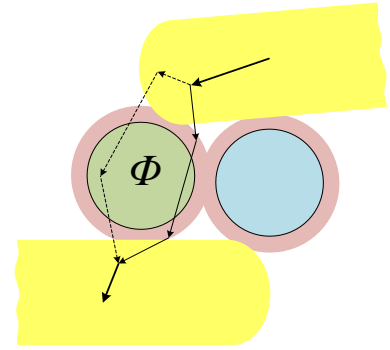

b

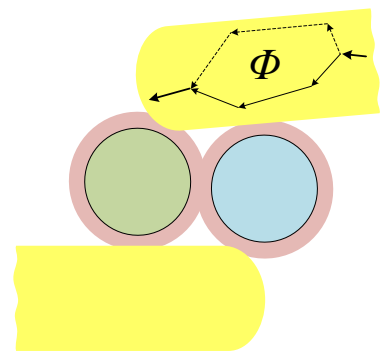

d

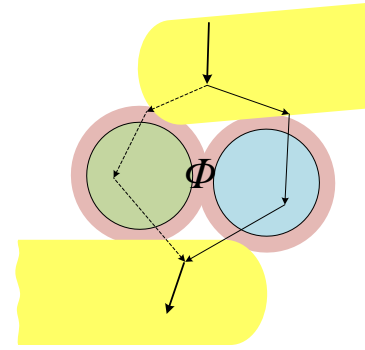

Au NP1 Au NP2

Figure 7.8. Schematics of the devices in Fig. 7.3 showing possible origin of the observed periodic oscillations in Fig. 7.5-7.7: UCF in leads and NRs (a), in a single Au NP (b), in areas containing single Au NP and molecular junction(s) (c); $A B$ effect (d). 
From the test measurements in Fig. 7.5 we may conclude that the observed interference effects are caused by the magnetic field piercing the device. There are two well-known interference effects at the mesoscopic scale: UCF and the AB effect (Sections 1.1-1.2). Figure 7.8 suggests the four possible scenarios which might cause oscillations in Figs. 7.5-7.7: 1 - UCF in leads and NRs (Fig. 7.8a); 2 - UCF in a single Au NP (Fig. 7.8b); 3 - UCF in areas containing a single Au NP and molecular junction(s) (Fig. 7.8c); 4 - AB effect (Fig. 7.8d). The period of observed oscillations $\Delta B$ varies between $\sim 4-6 \mathrm{~T}$ which corresponds to the enclosed area $S_{\text {in }}=(8.6 \pm 1.8) \cdot 10^{-16} \mathrm{~m}^{2}$ (assuming the circular area, the corresponding diameter is $23.3 \pm 2.4 \mathrm{~nm}$ ) for the $\mathrm{AB}$ effect and/or UCF for devices with small dimensions $\left(\ll l_{\varphi}\right.$, where $l_{\varphi}$ is the phase coherence length, which is in order of $1-2 \mu \mathrm{m}$ for gold [10]). Oscillations caused by UCF in NRs and leads (Fig. 7.8a) are expected to have much bigger

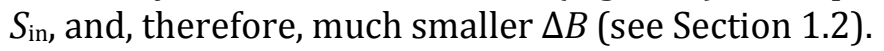

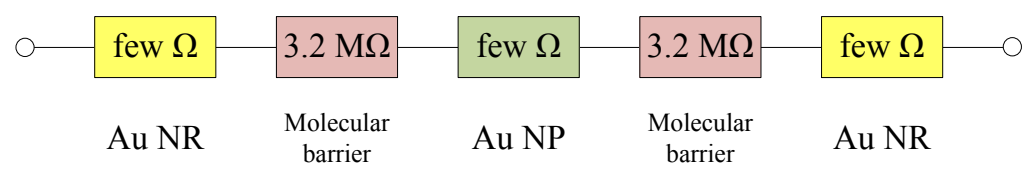

Figure 7.9. Block diagram of the electron transport through the Au NR molecular layer - Au NP - molecular layer - Au NR.

Figure 7.9 schematically represents the current circuit through the $\mathrm{Au}$ NR - molecular layer - Au NP - molecular layer - Au NR junction. Assuming that the observed oscillations are due to the interference effects in the metallic parts of the device, the visibility of the quantum oscillations (determined by the ratio of the oscillation signal to the main signal) would be:

$$
\frac{\Delta R}{R_{\Sigma}}=\frac{h / e^{2}}{R_{\mathrm{NR} 1}+R_{\mathrm{mb} 1}+R_{\mathrm{NP}}+R_{\mathrm{mb} 2}+R_{\mathrm{NR} 2}} \cong \frac{h / e^{2}}{2 R_{\mathrm{mb}}}=0.4 \%
$$

assuming that $R_{\mathrm{Au}}=R_{\mathrm{NR} 1}=R_{\mathrm{NR} 2}=R_{\mathrm{NP}}=\mathrm{a}$ few $\Omega$ and $R_{\mathrm{mb}}=R_{\mathrm{mb} 1}=$ $R_{\mathrm{mb} 2}=3.2 \mathrm{M} \Omega$, where $\Delta R=h / e^{2} \approx 25 \mathrm{k} \Omega$ is the quantum resistance, $R_{\Sigma}$ is the total resistance through the system, $R_{\mathrm{NR} 1}, R_{\mathrm{NR} 2}, R_{\mathrm{NP}}, R_{\mathrm{mb} 1}, R_{\mathrm{mb} 2}$ and $R_{\mathrm{Au}}$ are resistances of the first NR, the second NR, NP, the first molecular barrier, the second molecular barrier and gold, respectively. While the observed oscillations in Figs. 7.5-7.7 are $\sim 10 \%$ of the main signal. This is an indication that the observed amplitude of the oscillations is not due to the interference effects in the isolated metallic parts of the device, excluding options a $\mathrm{n} \mathrm{b}$ in Fig. 7.8, but more likely due to the presence of the tunnel barriers incorporated in the system [4]. 


\subsection{Hybrid Aharonov-Bohm Interferometers: Perspectives}

In this chapter we have shown that interference effects can be observed in inorganic bottom-up $\mathrm{Au} A \mathrm{~B}$ interferometers, and possibly also in hybrid inorganic-organic systems. The next step is to fabricate a hybrid $A B$ interferometer (where UCF and $\mathrm{AB}$ effect can be distinguished) with embedded molecular junctions in order to demonstrate coherent electron transport through organic materials. Figure 7.10 shows some proposed geometries for hybrid inorganic-organic $\mathrm{AB}$ interferometers.

An Au ring containing gaps functionalized with a desired SAM and bridged by Au NPs via DEP (Fig. 7.10a) is a good candidate for the hybrid inorganic-organic $\mathrm{AB}$ interferometers to study coherence in molecular layers. The main disadvantage of such interferometers is that at low temperatures, when interference effects are studied, electron transport is suppressed due to the Coulomb blockade in Au NPs acting as Coulomb islands which provides extra complications during measurements. To overcome the blockade one may study the interference effects in hot electrons by applying a bias voltage over the $\mathrm{AB}$ interferometer or adding side gates to independently control the Coulomb blockade of the dots. The $\mathrm{AB}$ effect has been exploited to demonstrate that electron tunnelling through a semiconductor quantum dot embedded in one arm of an $\mathrm{AB}$ ring is at least partly coherent $[11,12]$. A number of samples was tested. We measured clear UCF, but we did not observe an indication of the AB effect. It is possible that NP(s) in one branch of the ring is(are) coupled stronger to the leads than $\mathrm{NP}(\mathrm{s})$ in the other arm which may lead to conductance mostly through one branch of the $A B$ interferometer suppressing $\mathrm{AB}$ effect. We cannot demonstrate that NPs in both branches are touching the leads properly.

Coulomb blockade is a smaller issue in the $\mathrm{AB}$ rings fabricated using the wedging transfer technique (Fig. 7.10b). As discussed in chapter 3, besides the negligible effect of the Coulomb blockade molecular junctions created via wedging transfer method provide higher conductance (see Section 3.4) which may become a big advantage. Nevertheless, the alignment between the bottom and the top (transferred) electrodes is still a challenge. Figure 7.10c shows a proposed design of a hybrid $\mathrm{AB}$ ring (yellow and orange areas represent bottom and top electrodes, respectively) where the alignment problem is solved and by different ways of electrical contacting the number of molecular junctions in a device can be varied. Due to the low yield of the wedging transfer technique (Chapter 3), we did not succeed in the fabrication of hybrid $\mathrm{AB}$ rings with a reasonable conductance for molecular junctions $\left(<e^{2} / h\right)$ (Section 1.4). 
In chapter 4 we have shown that by selective chemical interactions $\mathrm{Au}$ NPs can be attached to Au NRs. We proposed to use the same technique to fabricate a hybrid $\mathrm{AB}$ interferometer by contacting NR-NR assemblies (Fig. 7.10d). The technique was shown to be very reproducible with a high yield $(>50 \%)$ and by exclusion of NPs from the assembly we avoid Coulomb blockade regime at low temperatures. In a few rings we observed strong UCF signal but no $\mathrm{AB}$ oscillations were present.

a

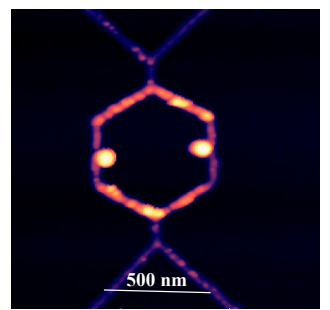

b

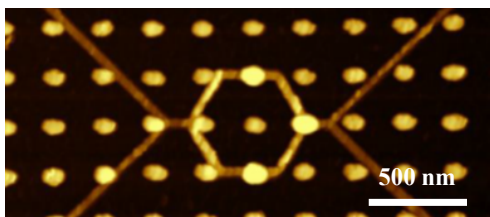

C
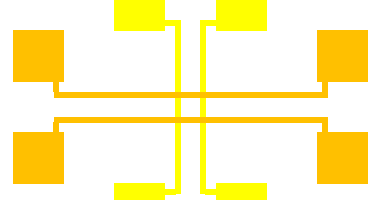

d

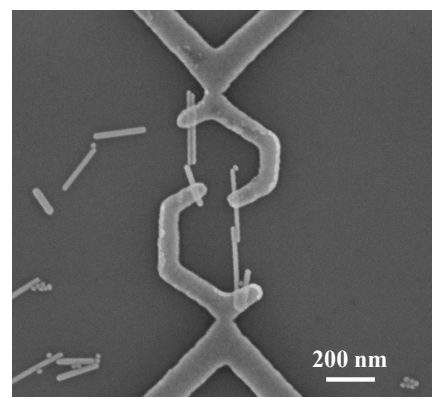

Figure 7.10. AFM and scanning electron microscopy images showing examples of the modified hybrid inorganic-organic $A B$ interferometers fabricated via (a) DEP, (b-c) wedging transfer technique and (d) bottomup assembled Au NRs assemblies. c, The design of the modified AB interferometer made via wedging transfer technique, where yellow and orange areas represent bottom and top (transferred) electrodes, respectively. 


\section{Conclusions}

We demonstrated the experimental observation of the $A B$ effect in an $\mathrm{Au}$ interferometer realized using the DEP method. Electrical measurements in a magnetic field through a pair of Coulomb islands in parallel decoupled from each other and from the $\mathrm{Au}$ NRs by a dithiol molecular layer reveal interference effects that might be addressed to the $\mathrm{AB}$ effect and as a consequence to coherent transport through the molecular layer. We proposed a few methods to fabricate hybrid inorganic-organic $A B$ interferometers where molecular layers of interest can be embedded for the consistent study of the coherent electron transport. The results suggest that the proposed novel hybrid $\mathrm{Au} A \mathrm{~B}$ interferometers are good candidates for a test-bed for studying coherent electron transport properties of organic molecules.

\section{REFERENCES}

[1] Y. Aharonov and D. Bohm, "Significance of Electromagnetic Potentials in the Quantum Theory," Physical Review, vol. 115, pp. 485-491, 08/01/ 1959.

[2] R. A. Webb, S. Washburn, C. P. Umbach, and R. B. Laibowitz, "Observation of h/e Aharonov-Bohm oscillations in normal-metal rings," Phys Rev Lett, vol. 54, pp. 2696-2699, Jun 241985.

[3] K. Kobayashi, H. Aikawa, S. Katsumoto, and Y. Iye, "ProbeConfiguration-Dependent Decoherence in an Aharonov-Bohm Ring," Journal of the Physical Society of Japan, vol. 71, pp. 2094-2097, 2002/09/15 2002.

[4] A. van Oudenaarden, M. H. Devoret, Y. V. Nazarov, and J. E. Mooij, "Magneto-electric Aharonov-Bohm effect in metal rings," Nature, vol. 391, pp. 768-770, 02/19/print 1998.

[5] S. Ballmann, R. Härtle, P. B. Coto, M. Elbing, M. Mayor, M. R. Bryce, et al., "Experimental Evidence for Quantum Interference and Vibrationally Induced Decoherence in Single-Molecule Junctions," Physical Review Letters, vol. 109, p. 056801, 07/30/ 2012.

[6] D. Fracasso, H. Valkenier, J. C. Hummelen, G. C. Solomon, and R. C. Chiechi, "Evidence for Quantum Interference in SAMs of Arylethynylene Thiolates in Tunneling Junctions with Eutectic Ga-In (EGaIn) TopContacts," Journal of the American Chemical Society, vol. 133, pp. 95569563, 2011/06/22 2011.

[7] C. M. Guedon, H. Valkenier, T. Markussen, K. S. Thygesen, J. C. Hummelen, and S. J. van der Molen, "Observation of quantum interference 
in molecular charge transport," Nat Nano, vol. 7, pp. 305-309, 05//print 2012.

[8] G. C. Solomon, C. Herrmann, T. Hansen, V. Mujica, and M. A. Ratner, "Exploring local currents in molecular junctions," Nat Chem, vol. 2, pp. 223-228, 03//print 2010.

[9] H. Valkenier, C. M. Guedon, T. Markussen, K. S. Thygesen, S. J. van der Molen, and J. C. Hummelen, "Cross-conjugation and quantum interference: a general correlation?," Physical Chemistry Chemical Physics, vol. 16, pp. 653-662, 2014.

[10] P. Mohanty, E. M. Q. Jariwala, and R. A. Webb, "Intrinsic Decoherence in Mesoscopic Systems," Physical Review Letters, vol. 78, pp. 3366-3369, 04/28/ 1997.

[11] A. Yacoby, M. Heiblum, D. Mahalu, and H. Shtrikman, "Coherence and Phase Sensitive Measurements in a Quantum Dot," Physical Review Letters, vol. 74, pp. 4047-4050, 05/15/ 1995.

[12] K. Kobayashi, H. Aikawa, A. Sano, S. Katsumoto, and Y. Iye, "Fano resonance in a quantum wire with a side-coupled quantum dot," Physical Review B, vol. 70, p. 035319, 07/27/ 2004. 


\title{
Appendix A
}

\author{
Stability Diagrams of \\ Single-Electron Transport through a \\ Pair of Coulomb Islands Coupled in Parallel
}




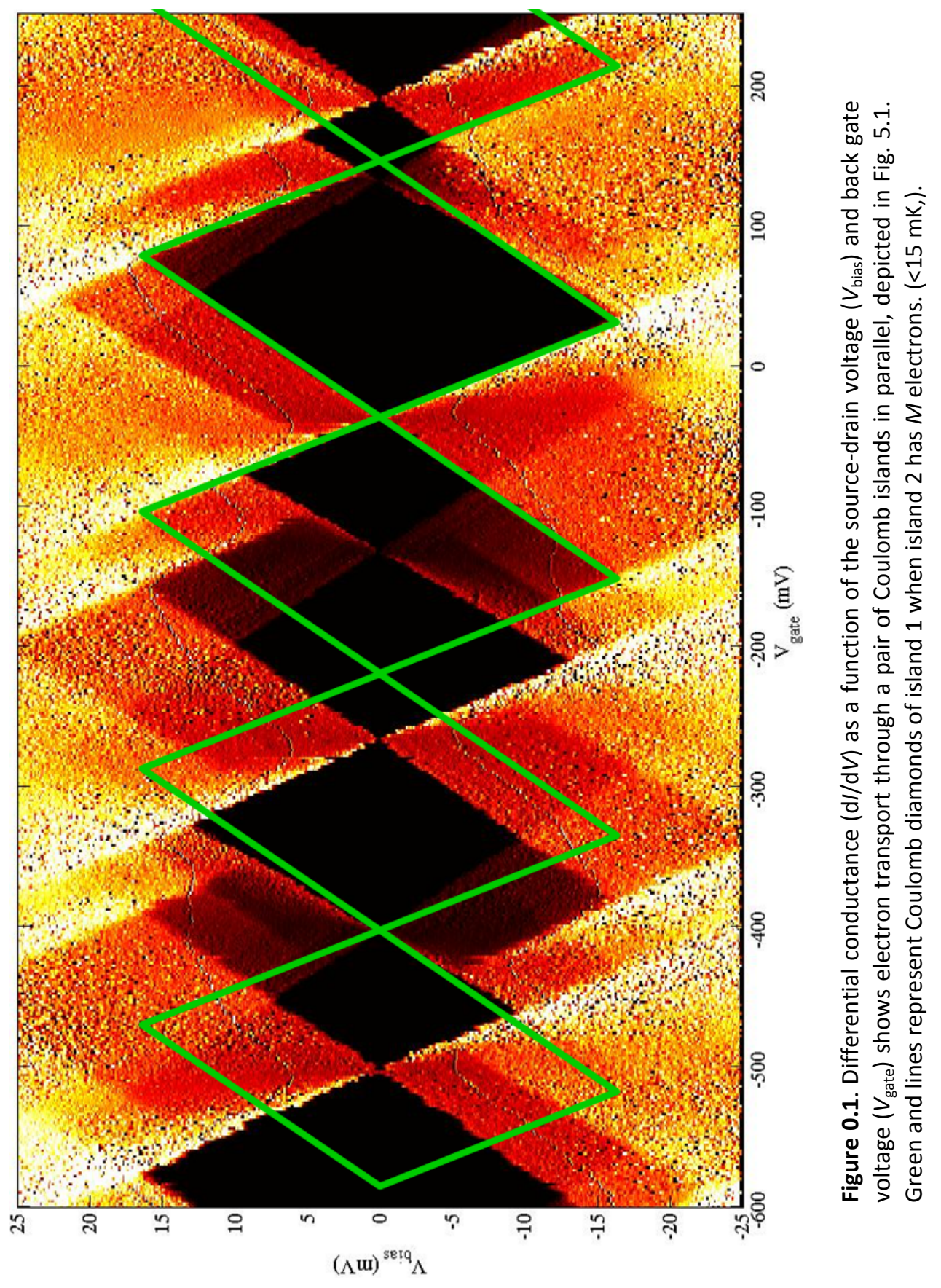




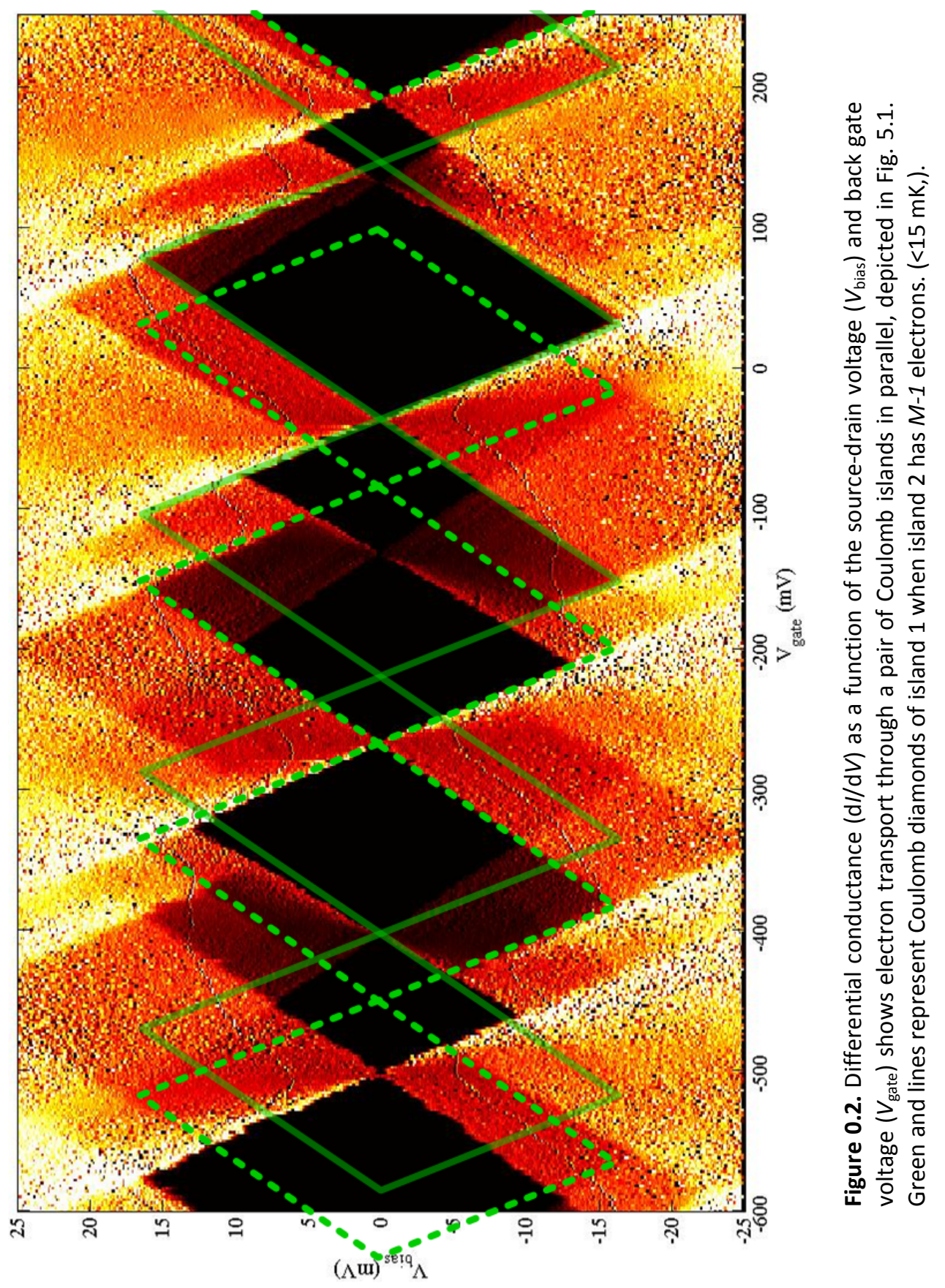




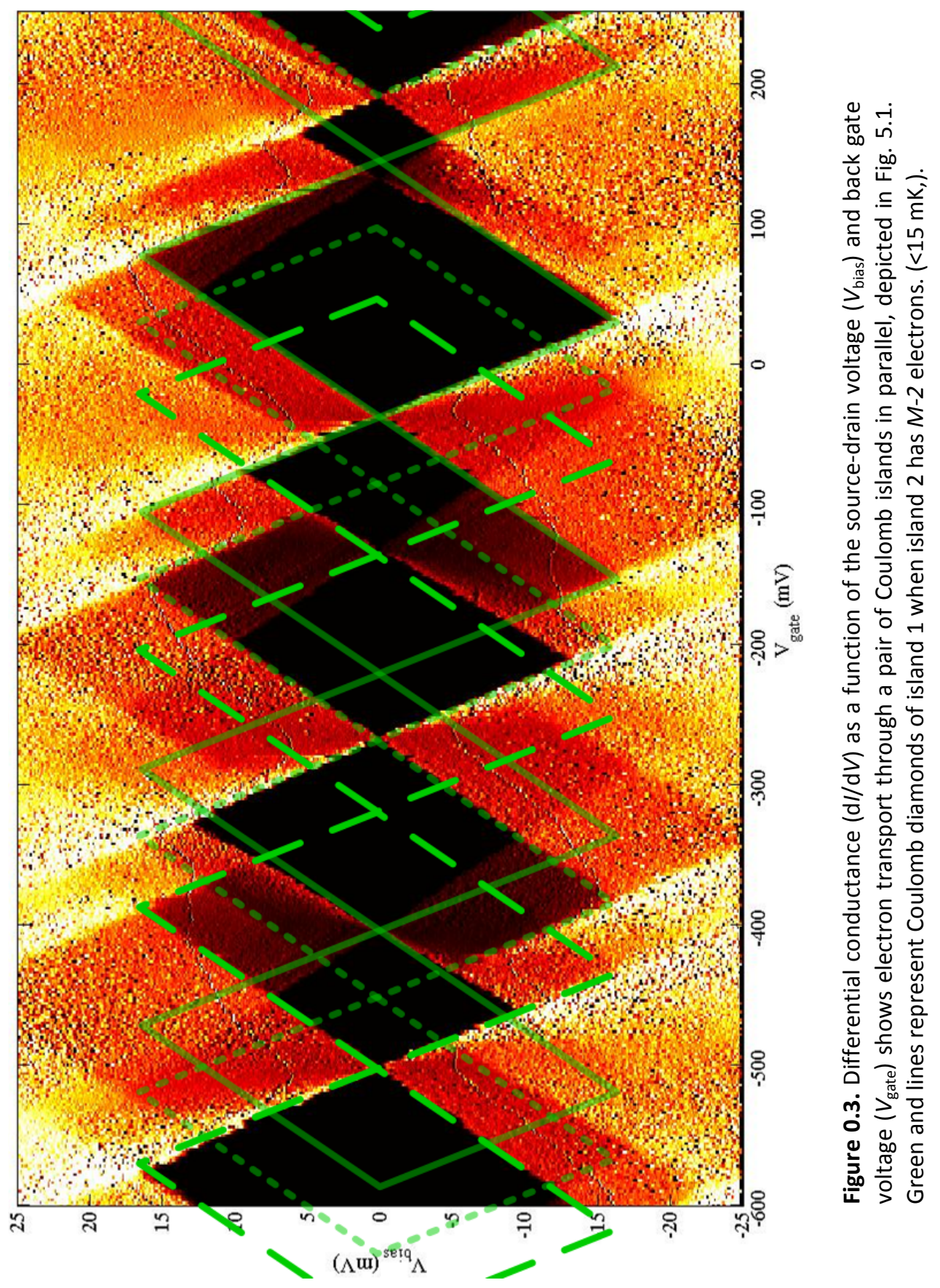




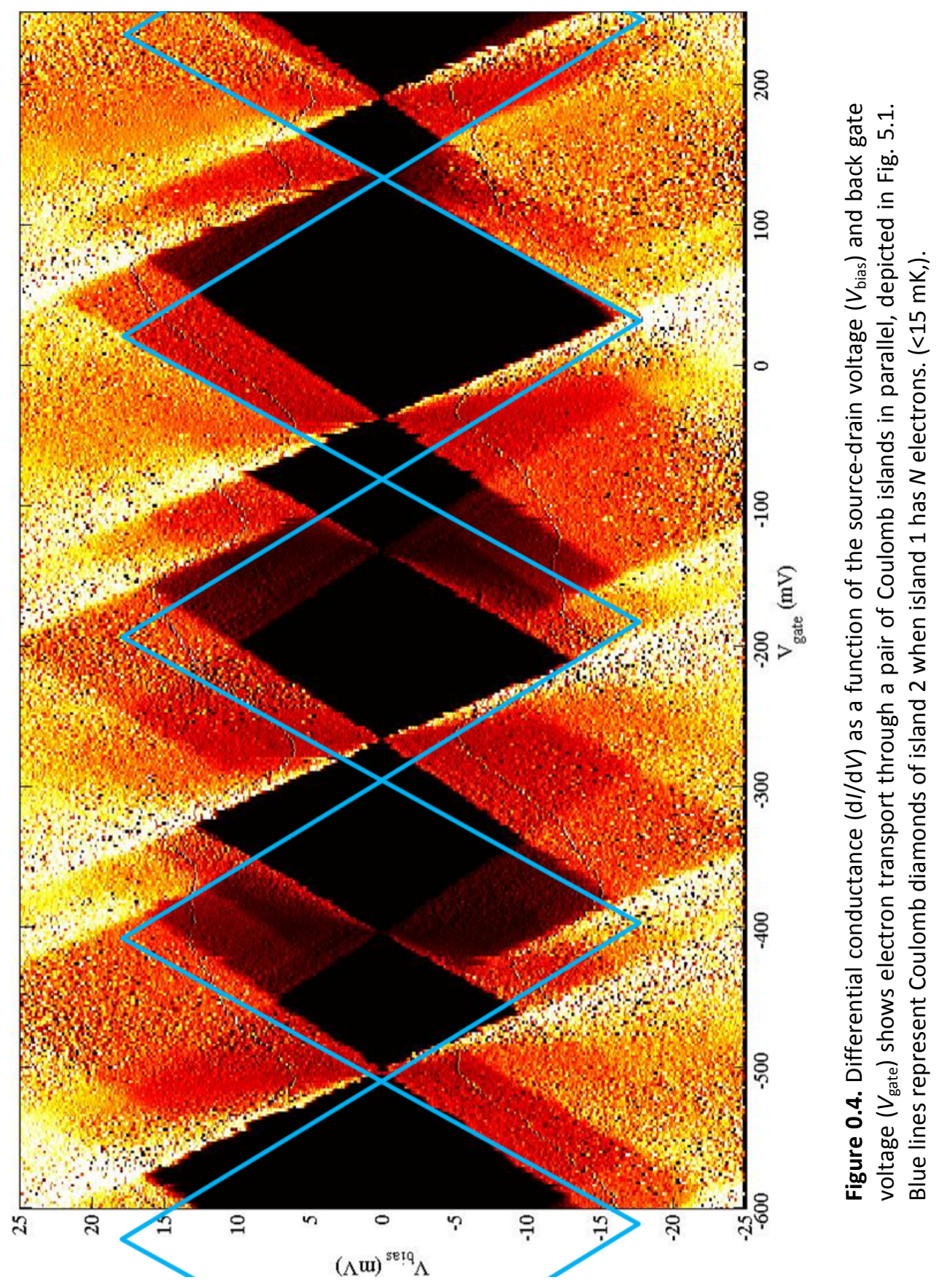




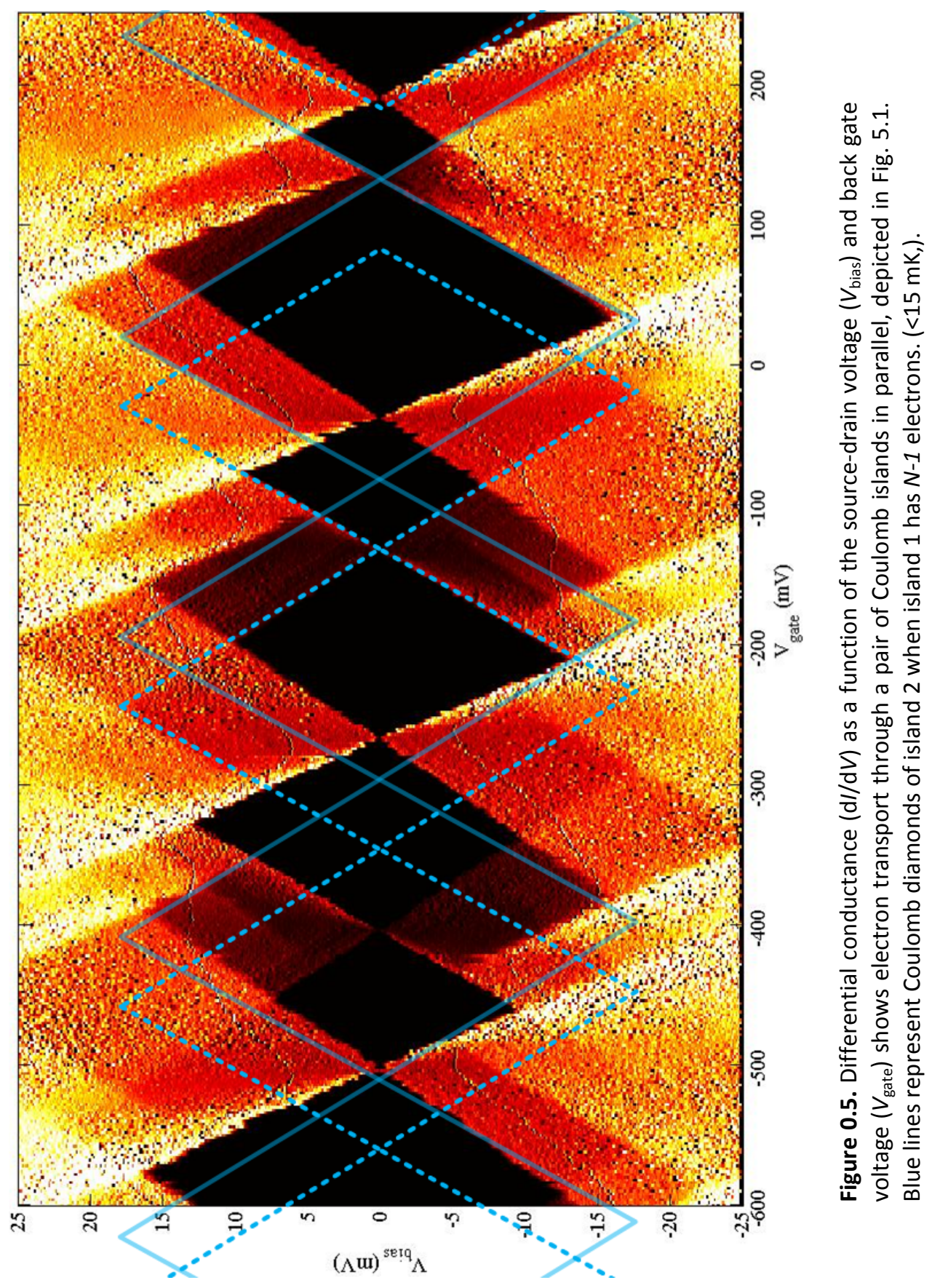




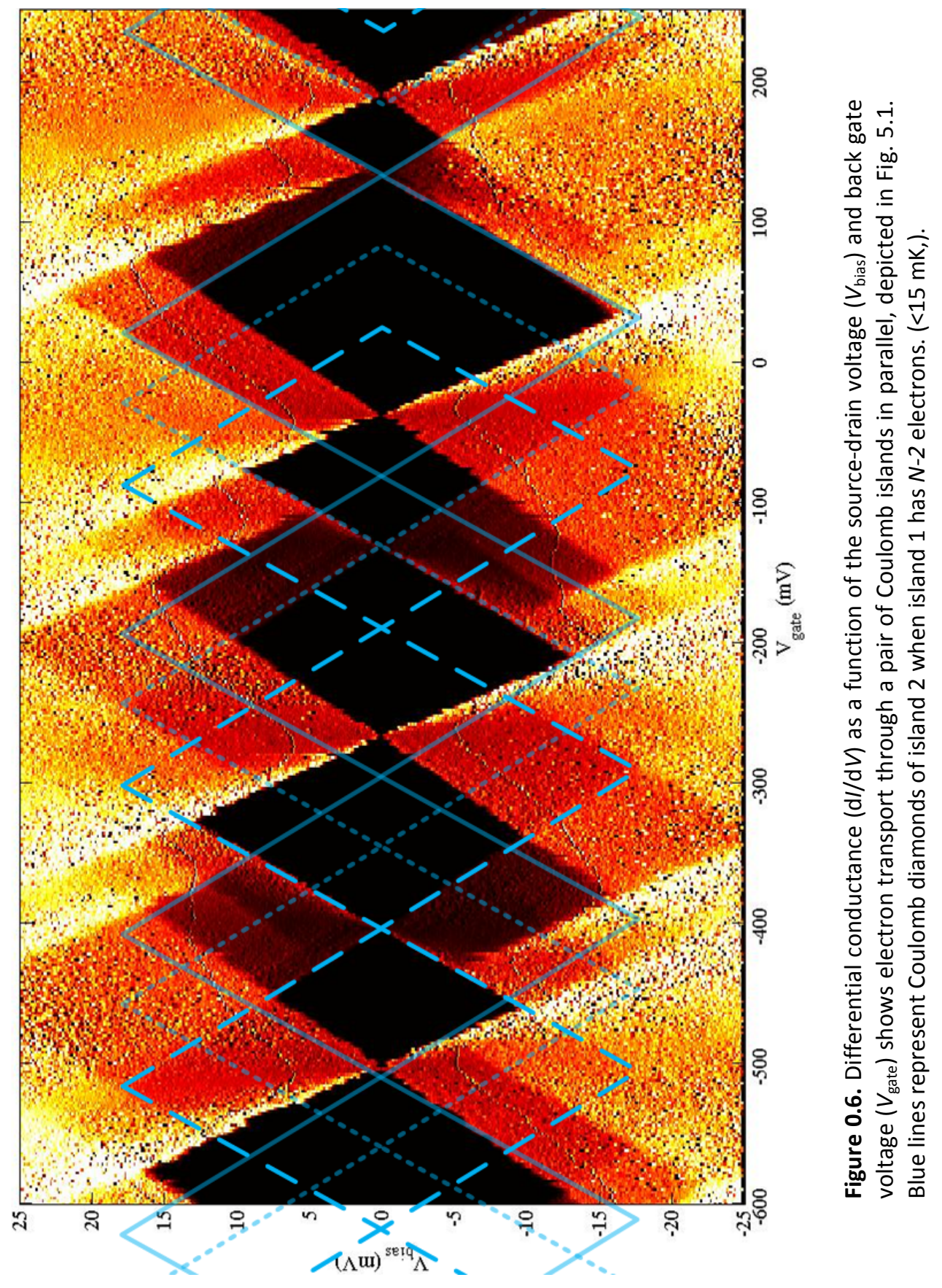




\section{Summary}

\section{Charge Transport in Bottom-Up Inorganic-Organic and Quantum-Coherent Nanostructures}

This thesis is based on results obtained from experiments designed for a consistent study of charge transport in bottom-up inorganic-organic and quantum-coherent nanostructures. New unconventional ways to build elements of electrical circuits (like dielectrophoresis, wedging transfer and bottom-up fabrication using selective chemical interactions) were studied and are presented in this thesis. We also investigated the next steps in understanding fundamental physics phenomena such as charge transport through a pair of metallic Coulomb islands coupled in parallel, coherent transport through organic molecular monolayers and dynamical nonlocality in normal metals.

Dielectrophoresis (DEP) and wedging transfer are top-down techniques which allow soft-contacting of electrodes covered by selfassembled molecular monolayers (SAMs) without damaging it, leading to the formation of the $\sim 1-2 \mathrm{~nm}$ wide molecular tunnelling junctions avoiding the requirement for the creation of extremely small nanogaps. DEP is a high-yield, simple and reproducible processing technique that is a good alternative topdown technology for building of molecular bridges. This method allows not only characterisation of a large number of molecular monolayers, but also fabrication of single-electron transistors (SETs) by trapping a single nanoparticle (NP) (metallic or semiconducting) between source, drain and gate electrodes and decoupling the NP via molecular barriers. Wedging transfer is a promising alternative method for the fabrication of nano- and micro-sized area molecular junctions. However, the low-yield of the working devices fabricated by means of wedging transfer indicates the necessity of further improvement of the fabrication process.

The study of molecular SAMs (Chapters 2,3) shows that electron transport through alkanedithiols is typical of non-resonant tunnelling, independent of the temperature or external magnetic field. However, the molecular length as well as the molecular composition strongly affect the 
conductance through molecules. As predicted, alkanedithiols are more resistive than $\pi$-conjugated molecular monolayers.

On-going miniaturization of silicon transistors over the last few decades, driven by scientific and technological innovations, is enabled by developments in top-down fabrication methods. Nevertheless, approaching of crucial features below $\sim 10 \mathrm{~nm}$ makes top-down methods more challenging and costly. This raises the interest in the investigation of a new unconventional way of realizing functional nanoelectronic devices. In chapter 4 a bottom-up approach for fabrication of SETs based on single $\sim 20 \mathrm{~nm} \mathrm{Au} \mathrm{NP}$ is exploited. We successfully combined self-assembly of an $\mathrm{Au} N \mathrm{NP}$ to $\mathrm{Au}$ nanorods (NRs) with top-down lithography. Low-temperature electrical measurements of the electron transport through the SETs revealed clear signatures of the classical Coulomb blockade regime. The fabrication process shows very high reproducibility $(>50 \%)$ and the SETs are very stable over a few months. Both the temperature and size dependence are in good agreement with theoretical predictions.

Electron transport measurements through a pair of metallic Coulomb islands coupled in parallel (Chapter 5) show that there is strong electrostatic interaction between the islands as they are in close proximity to each other. This capacitive cross talk leads to a rich charge stability diagram.

Nonlocality is a key feature discriminating classical and quantum physics. In chapter 6 , we demonstrate a systematic study of the nonlocal electron transport through Aharonov-Bohm (AB) interferometers showing a new aspect of the dynamical nonlocality in a quantum network. Our experimental foundations are supported by a theoretical model that explains the fundamental nature of the dynamical delocalization. Dynamical nonlocality is not limited by the diffusive transport regime or by the specific wave-particle used and damps away with the reduction of the amount of conduction channels.

Finally, in chapter 7, we demonstrate an evidence of coherent charge transport through a1,8-alkanedithiol monolayer embedded as molecular barrier in a system of a pair of Coulomb islands coupled in parallel and working as two parallel SETs. Understanding to what extent transport through organic molecular monolayers is coherent and how the coherence depends on the molecule's characteristics and/or external parameters is a fascinating question raised in recent years due to the potential application of molecular monolayers in nanoelectronics.

Ksenia S. Makarenko

Enschede, May 2015 


\section{Samenvatting}

\section{Ladingstransport in "Bottom-Up" Inorganische-Organische en Kwantum-Coherente Nano-Structuren}

Dit proefschrift is gebaseerd op resultaten die verkregen zijn uit experimenten ontworpen voor het bestuderen van ladingstransport in "bottom-up" inorganische-organische en kwantum-coherente nano-structuren. Nieuwe onconventionele manieren om elementen van een elektrisch circuit te maken (zoals diëlektroforese, "wedging transfer" en bottom-up fabricage waarbij gebruik gemaakt wordt van selectieve chemische interacties) werden bestudeerd en worden gepresenteerd in dit proefschrift. We onderzochten ook stappen in het begrijpen van fundamentele fysische verschijnselen zoals ladingstransport door een tweetal parallel geschakelde metalen Coulomb eilanden, coherent ladingstransport door organische moleculaire monolagen en dynamische non-lokaliteit in metalen.

Diëlektroforese ("dielectrophoresis", DEP) en wedging transfer zijn top-down technieken die soft-contact van elektroden onder zelf geassembleerde moleculaire monolagen (SAMs) toelaten zonder deze lagen te beschadigen, wat leidt tot de formatie van $\sim 1-2 \mathrm{~nm}$ brede moluculaire tunnelcontacten zonder de noodzaak voor extreem smalle nano-kanalen. DEP is een simpele en reproduceerbare techniek met een hoog rendement wat het een goed alternatief maakt voor het fabriceren van moleculaire bruggen. Deze methode laat niet alleen het karakteriseren van een groot aantal moleculaire monolagen toe, maar ook de fabricage van single-electron transistors (SETs) door het vangen van enkele nano-deeltjes ("nanoparticles", NPs) (metallisch of halfgeleider) tussen elekrodes en het ontkoppelen van deze NPs via moleculaire barrières. Wedging transfer is een veelbelovend alternatief voor de fabricage van moleculaire juncties op nano- en microschaal. De lage hoeveelheid werkende devices die met wedging transfer gemaakt werden, is een indicatie voor de noodzaak voor verdere verbetering van dit fabricageproces.

De studie van moleculaire SAMs (hoofdstuk 2.3) laat zien dat elektronentransport door alkaandithiolen een typisch voorbeeld is van nietresonante tunnelling, onafhankelijk van de temperatuur of het magnetische 
veld. De moleculaire lengte en de moleculaire compositie hebben een sterke invloed op de geleiding van de moleculaire juncties. Zoals verwacht resulteren de alkaandithiolen in een hogere weerstand dan $\pi$-geconjugeerde moleculaire monolagen.

De continue miniaturisatie van silicium transistoren in de afgelopen decennia, die gedreven wordt door wetenschappelijke en technologische innovaties, wordt mogelijk gemaakt door de ontwikkelingen in top-down fabricagemethoden. Echter, het benaderen van afmetingen onder de $\sim 10 \mathrm{~nm}$ maakt top-down methodes moeilijker en duurder. Dit motiveert onderzoek naar nieuwe onconventionele manieren voor het realiseren van functionele nano-elektrische componenten. In hoofdstuk 4 wordt een bottom-up benadering voor de productie van SETs op basis van enkele $\sim 20 \mathrm{~nm} \mathrm{Au} \mathrm{NP}$ geëxploiteerd. Met succes hebben wij zelfassemblage van $\mathrm{Au}$ NPs met $\mathrm{Au}$ "nanorods" (NRs) gecombineerd met top down lithografie. Elektrische metingen van elektronentransport door de SETs bij lage temparatuur laten een duidelijke signatuur zien van klassieke Coulomb-blokkade. Het fabricageproces heeft een zeer hoge reproduceerbaarheid $(>50 \%)$ en de SETs zijn stabiel gedurende enkele maanden. De temperatuurs- en grootteafhankelijkheid zijn in goede overeenkomst met de theoretische voorspellingen.

Metingen van het elektronentransport door een paar parallel geschakelde metalen Coulomb eilanden (hoofdstuk 5) laten zien dat er een sterke elektrostatische interactie is tussen de eilanden. Deze capacitieve koppeling leidt tot een rijk ladingstransportdiagram.

Niet-lokaliteit is een belangrijk kenmerk voor het onderscheiden van klassieke- en kwantumfysica. In hoofdstuk 6 beschrijven we een systematische studie van het niet-lokale elektronentransport door AharonovBohm ( $\mathrm{AB}$ ) interferometers die een nieuw aspect van de dynamische nietlokaliteit in een kwantum netwerk laten zien. Onze experimenten worden ondersteund door een theoretisch model dat het fundamentele karakter van de dynamische delokalisatie verklaart. Dynamische niet-lokaliteit wordt niet beperkt door het diffusieve transport of door het specifieke deeltje(-golf) dat wordt gebruikt en vermindert met de hoeveelheid geleidende kanalen.

Tenslotte laten we in hoofdstuk 7 een indicatie zien voor coherent ladingstransport door een 1,8-alkaandithiolmonolaag ingebed als een moleculaire barrière in een systeem van een tweetal parallel geschakelde Coulomb eilanden en werkend als twee parallele SETs. In hoeverre transport door organische moleculaire monolagen coherent is en hoe de coherentie afhankelijkheid is van de moleculaire karakteristieken en/of de externe parameters is een fascinerende vraag die opgekomen is in de laatste jaren 
vanwege de mogelijkheid om moleculaire monolagen te gebruiken in nanoelektronica.

Ksenia S. Makarenko

Enschede, May 2015 



\section{Acknowledgements}

This thesis is a result of four years of experimental research on the charge transport in bottom-up inorganic-organic and quantum-coherent nanostructures. For me it was, definitely, four great, wonderful years. The four years of hard and interesting work as well as meeting new people, some of them have changed my life $-\odot$. But it would not be even possible to do this work without my Ph.D. adviser Wilfred G. van der Wiel, who gave me an opportunity to join NanoElectronics group as a Ph.D. researcher on the $1^{\text {st }}$ of April 2011, mentioning that it was not a joke. Wilfred, thank you for being a great guide during last four years. I am thankful to you for being patient and never giving strong suggestions and/or judgments and letting your students come to the right answers and conclusions themselves as well as work independently, motivated by their scientific "hunger" for knowledge. I am very glad that I met you not only as a scientist but also as a person. I hope that I will have many chances in future to ask you "a small question" :).

Of course, I thank my three very important collaborators - my three "partners in crime": Dr. S.K. Bose, Dr. Z. Liu and Dr. E. Strambini. It was my real pleasure to work with you. Our teamwork gave us very good harvest in a shape of great results. Elia, you introduced me to the world of cryogenic temperatures and low noise. Thank you for sharing your experience and your knowledge with me. Zhihua, you did try to kill my fear of chemistry, not hundred percent successfully, but, thank to you, I could manage to deal with it. Saurabh, you were my colleague, my office buddy and became my good friend. Thank you for a very nice trading system, when a few hours of EBLing could be exchanged to a few layers of freshly evaporated pure gold.

Unfortunately, in the absence of Wilfred, Michel P. de Jong had to take all the bullets from me. Thank you for trying to help me with answering all the questions, most of the times completely unrelated to your research field. When the time came to Coulomb blockades, Coulomb islands and everything what can be combined with "Coulomb", Floris A. Zwanenburg, thank you for very useful discussions.

The work in any of our labs is just impossible without Thijs Bolhuis, Johnny G.M. Sanderink and Martin H. Siekman. Thijs, thank you very much for all your help in the "coolest" place in Twente. Especially, being with me during the magnet quench $\odot$. Johnny, thank you for meters of evaporated gold and scanned samples. Martin, you know, that the AFM lab is "crying" without you. 
Thank you very much not only for your help with electronics but also for our "non-systematic" jogging during the lunch breaks, skiing and "colour-run". That was awesome!

Karen and Susan (Susie), you two are just wonderful! You are nice to look at, nice to talk to, nice to work with. When you laugh, all building is laughing with you. Together with Niels and Erwin, the "NE-coffee"-lovers, you create a nice and friendly atmosphere for the whole corridor. Thank you very much for your constant positive attitude! I am very glad that I met you.

Janine and Matthias, guys, your help in the labs was boundless. Janine, thank you for everything, for the help in the chemical lab, in the cleanroom and for our break exercises when we were using cryogenic systems as gym equipment $:-$. Matthias, thank you for help in the lab in the evenings, on weekends, with trying to understand the physics behind the measurements, for the stolen white-board, for the "Hawaii party" and of course for all the support at crucial moments.

Gulbostan, it was my pleasure to meet you as a friend and to work together with you during your lab course and on your Master-thesis. I hope, there will be a chance to meet you again!

I would like to thank Ina for our teamwork on nanoparticles networks and teaching me how to get the "precise" molecular concentration in a solution (-). I am also grateful to Heiko Wolf and Mona Klein (r.i.p.) for our short collaboration on NR-NP-NR assemblies. Sadly, you never know when and how your research can be interrupted.

My dear Julia, Derya and Janine! I guess, I do not have to say anything; you know how I feel about you. That was the greatest girls' office ever! We went along through all our happy and sad moments. Thank you for the time spent together. I am very happy that we could become more than colleagues. We had a great time!

Matthias, Elmer and Chris, the "party guys", you were my chief advisors for the "avond uitjes". Thank you for the spent time and "forcing" me sometimes to relax from the lab work. "Doing a Ph.D. is a lifestyle". Joost and Sergei, thank you, for many discussions we had. Joost, please, take care of our Journal Club $:$.

A good work could not be done without a very good environment within the group that was "sponsored" by the input of every present and former NE-member. Hajo, Tian, Serkan, Rabindra, Johnny W, Wen, Lan Anh, Peter, Bernardus, Lars (TST), Filipp, Bojian, Kai, Liang Y, Hao, Frank, Liang D, Michel Z, Jelmer, Celestine, Dilu, Christoph, Tamer, Francesca, Reinier, Loek, Joren, Yizhen, Joost H, Bjorn thank you all for good atmosphere in the group. 
I would like to acknowledge all clean room staff, especially, Hans, Huib, Rene and Peter for your help, advise and for keeping the lab running. Edin, Kees $C$ and Chris, I cannot imagine the clean room without you! Thank you not only for your help but also for creating a great clean environment and cheering me up!

From the first days of staying in Enschede I was taken under the control of the Russian mafia: Anja\&Victor, Anton\&Yulia, Andrej, Olja, Sasha, Nuno, Robin\&Mafi, Thomas\&Magda, Zhare, thank you very much for your friendship!

And of course thank you Marco\&Sabrina, Tieskuh\&Eliana, Theo\&Marta, Theo\&Elea, Kirill\&Svetlana, and Master Thomas. We had a lot of fun moments!

Mishka and Mishechek, I guess, no words have to be said. Too many things connect us. I am unbelievably lucky person that I have you as my best friends. You have become more than friends, you are like brothers to me.

My staying in the The Netherlands could not be the same if I would not have you, familie Vergeer. I am deeply grateful to you for giving me a second "home". Helene and Paul, you are wonderful people, thank you for everything you have done for me!

Kurtechek, the most physics discussions I had with you, most of the times on holidays or during a "romantic" dinner, when many schematics were drawn on a piece of a napkin. During my Ph.D. you had to endure most. I am very happy that I was "forced" by Wilfred to work with you (-).

The last but not least, I would like to thank my family. Without all of you, my mommy, Dashulja, grandpa, granny and Gennady, I would never be able to do this work. All your support and love helped me along the long way to my promotion day. I missed you most! Papa, I wish you would be here with me. I hope I could make you be proud. You will always be in my heart and my thoughts. Love you.

Ksenia S. Makarenko

Enschede, May 2015 


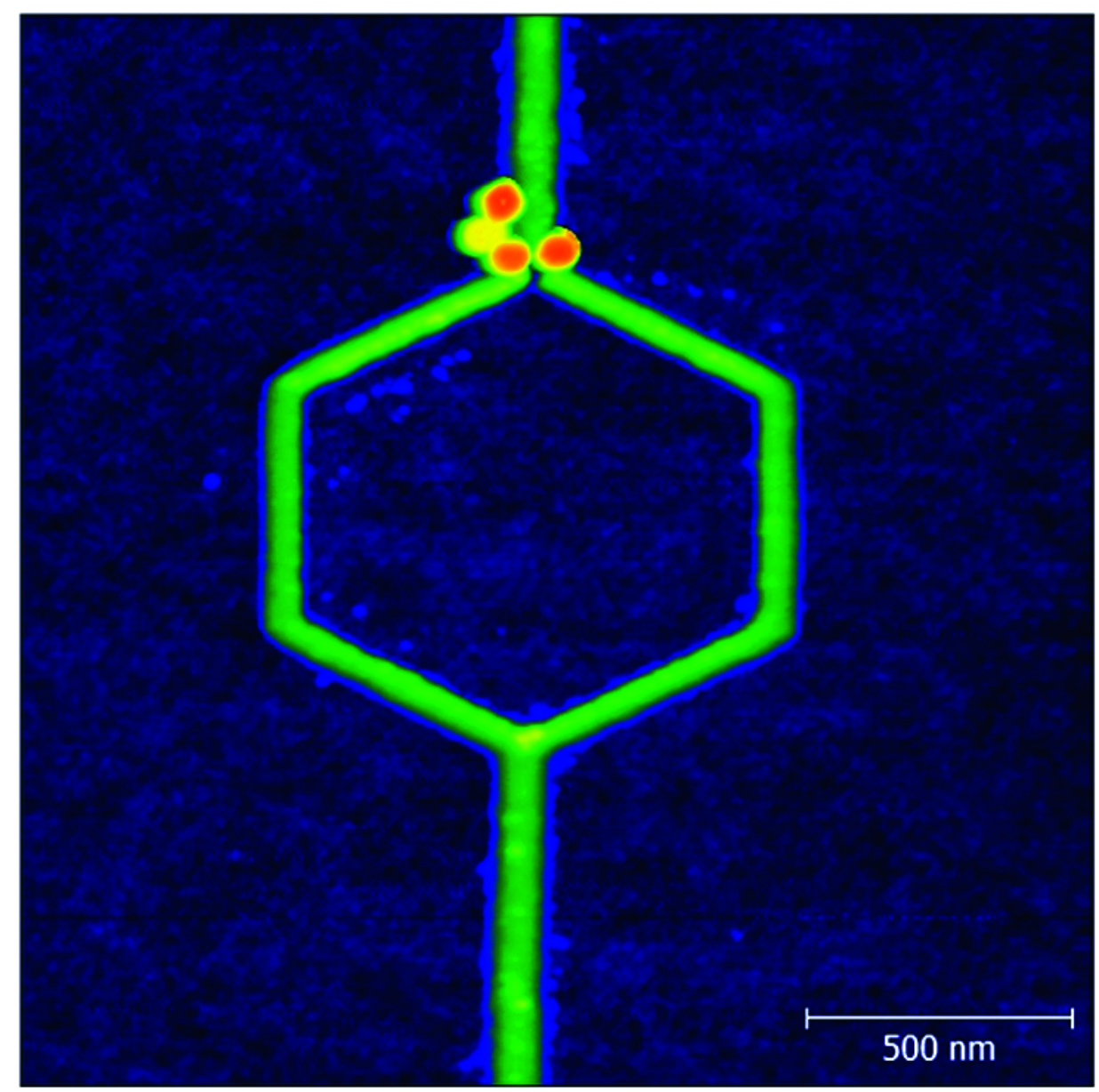

Figure LXXVI. Atomic force microscopy image of a Au Aharonov-Bohm interferometer fabricated on top of the $\mathrm{p}++\mathrm{Si}$ substrate with thermally grown $35 \mathrm{~nm}$ think $\mathrm{SiO}_{2}$ layer. Interferometer contains a gap functionalised by 1,6-octanedithiol self-assembled monolayer and bridged by $60 \mathrm{~nm}$ Au nanoparticles. Image made by Martin Siekman. 